\title{
Derivation of the Time Dependent Gross-Pitaevskii Equation in Two Dimensions
}

\author{
Maximilian Jeblick $^{1}$, Nikolai Leopold ${ }^{2}$ (D) Peter Pickl $^{3,4}$ (D) \\ 1 Mathematisches Institut, Ludwig-Maximilians-Universität München, Theresienstraße 39, 80333 Munich, \\ Germany. E-mail: maximilian.jeblick@khumbu.ai \\ 2 Institute of Science and Technology Austria (IST Austria), Am Campus 1, 3400 Klosterneuburg, Austria. \\ E-mail: nikolai.leopold@ist.ac.at \\ 3 Duke Kunshan University, Duke Avenue 8, Kunshan 215316, China. E-mail: peter.pickl@ dukekunshan.edu.cn \\ 4 Ludwig-Maximilians-Universität München, Theresienstraße 39, 80333 Munich, Germany. \\ E-mail: pickl@math.lmu.de
}

Received: 7 December 2017 / Accepted: 9 September 2019

Published online: 8 November 2019 - (C) The Author(s) 2019

\begin{abstract}
We present microscopic derivations of the defocusing two-dimensional cubic nonlinear Schrödinger equation and the Gross-Pitaevskii equation starting from an interacting $N$-particle system of bosons. We consider the interaction potential to be given either by $W_{\beta}(x)=N^{-1+2 \beta} W\left(N^{\beta} x\right)$, for any $\beta>0$, or to be given by $V_{N}(x)=e^{2 N} V\left(e^{N} x\right)$, for some spherical symmetric, nonnegative and compactly supported $W, V \in L^{\infty}\left(\mathbb{R}^{2}, \mathbb{R}\right)$. In both cases we prove the convergence of the reduced density corresponding to the exact time evolution to the projector onto the solution of the corresponding nonlinear Schrödinger equation in trace norm. For the latter potential $V_{N}$ we show that it is crucial to take the microscopic structure of the condensate into account in order to obtain the correct dynamics.
\end{abstract}

\section{Contents}

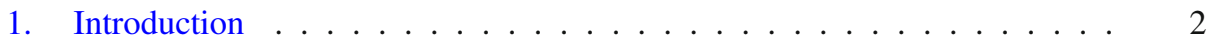

2. Main Result . . . . . . . . . . . . . . . . . . . . . . . . 6

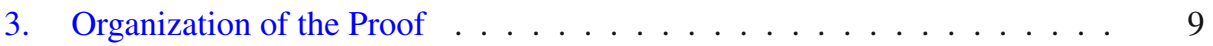

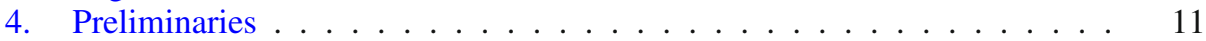

5. Microscopic Structure in 2 Dimensions . . . . . . . . . . . . . . . 17

5.1 The scattering state . . . . . . . . . . . . . . . . . . . . 17

5.2 Properties of the scattering state . . . . . . . . . . . . . . 19

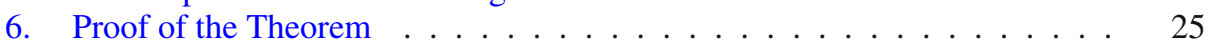

6.1 Proof for the NLS scaling $W_{\beta}$ with $\beta>0 \ldots \ldots . \ldots . . . . . .25$

6.1.1 Definition of the functional . . . . . . . . . . . . . 25

6.1.2 Preliminaries for the Grönwall estimate . . . . . . . . . . . . 27

6.1.3 The Grönwall estimate . . . . . . . . . . . . . . . . . . 29

6.2 Proof for the exponential scaling $V_{N} \ldots \ldots \ldots . \ldots . \ldots 31$

6.2.1 Definition of the functional . . . . . . . . . . . . 31

6.2.2 Preliminaries for the Grönwall estimate . . . . . . . . . . . . . . 34 
6.2.3 The Grönwall estimate . . . . . . . . . . . . . . . . . . 36

7. Rigorous Estimates . . . . . . . . . . . . . . . . 37

7.1 Smearing out the potential $w_{\beta} \ldots \ldots \ldots \ldots \ldots \ldots$

7.2 Estimates on the cutoff . . . . . . . . . . . . . . . 40

7.3 Proof of Lemma $6.6 \ldots \ldots$

7.4 Proof of Lemma $6.13 \ldots \ldots$. . . . . . . . . . . . . . 52

7.5 Energy estimates $\ldots \ldots \ldots \ldots \ldots \ldots \ldots$

\section{Introduction}

We are interested in the time evolution of bosonic quantum systems of $N$ particles in two dimensions that interact with each other by a two-particle interaction potential. At a given time $t$, the state of the system is described by a wave function $\Psi_{t} \in L_{s}^{2}\left(\mathbb{R}^{2 N}, \mathbb{C}\right)$, where $L_{s}^{2}\left(\mathbb{R}^{2 N}, \mathbb{C}\right)$ denotes the Hilbert space of all $\Psi \in L^{2}\left(\mathbb{R}^{2 N}, \mathbb{C}\right)$ which are symmetric under permutations of the variables $x_{1}, \ldots, x_{N} \in \mathbb{R}^{2}$. The Hamiltonian of the system is given by

$$
H_{U}=-\sum_{j=1}^{N} \Delta_{j}+\sum_{1 \leq j<k \leq N} U\left(x_{j}-x_{k}\right)+\sum_{j=1}^{N} A_{t}\left(x_{j}\right)
$$

with $A .: \mathbb{R}^{2} \times \mathbb{R} \rightarrow \mathbb{R}$ being a time-dependent external potential and $U: \mathbb{R}^{2} \rightarrow \mathbb{R}$ modeling the interaction between the particles. The time evolution of the system is described by the Schrödinger equation

$$
\mathrm{i} \partial_{t} \Psi_{t}=H_{U} \Psi_{t}
$$

with initial datum $\Psi_{0} \in L_{s}^{2}\left(\mathbb{R}^{2 N}, \mathbb{C}\right)$. In general, even for small particle numbers $N$, it is not possible to solve the Schrödinger equation exactly or numerically. The time evolution of the system, however, can approximately be determined if one studies special classes of initial conditions and certain types of interaction potentials. In this paper, we are concerned with the dynamical evolution of a Bose-Einstein condensate. This state of matter appears if one cools bosons in an external trapping potential near absolute zero temperature such that almost all particles occupy the same quantum state (see e.g. [38] for a comprehensive discussion). After the trapping potential has been changed or completely switched off, the condensate is no longer in equilibrium and one would like to study its evolution in space.

Mathematically, the appearance of a Bose-Einstein condensate is described by means of the one-particle reduced density matrix $\gamma_{\Psi}^{(1)}$ of the state $\Psi \cdot \gamma_{\Psi}^{(1)}$ is a non-negative trace class operator on $L^{2}\left(\mathbb{R}^{2}, \mathbb{C}\right)$ with an integral kernel given by

$$
\gamma_{\Psi}^{(1)}\left(x, x^{\prime}\right)=\int_{\mathbb{R}^{2 N-2}} \overline{\Psi\left(x, x_{2}, \ldots, x_{N}\right)} \Psi\left(x^{\prime}, x_{2}, \ldots, x_{N}\right) d^{2} x_{2} \ldots d^{2} x_{N} .
$$

A state $\Psi$ is said to exhibit complete Bose-Einstein condensation, if there exists a oneparticle wave function $\varphi \in L^{2}\left(\mathbb{R}^{2}, \mathbb{C}\right)$ with $\|\varphi\|=1$ such that $\gamma_{\Psi}^{(1)} \rightarrow|\varphi\rangle\langle\varphi|$ in trace norm as $N \rightarrow \infty .{ }^{1}$ Initially, we consider a complete condensed state $\Psi_{0}$ and then show

\footnotetext{
${ }^{1}$ We like to remark that it is well known that the convergence of $\gamma_{\Psi}^{(1)}$ to $|\varphi\rangle\langle\varphi|$ in trace norm is equivalent to the respective convergence in operator norm since $|\varphi\rangle\langle\varphi|$ is a rank-1-projection, see Remark 1.4. in [51]. For other indicators of condensation and their relation we refer to [41].
} 
that $\gamma_{\Psi_{t}}^{(1)} \rightarrow\left|\varphi_{t}\right\rangle\left\langle\varphi_{t}\right|$ as $N \rightarrow \infty$, where $\varphi_{t}$ solves a nonlinear Schrödinger equation. This statement shows that the condensate is stable during the time evolution. Moreover, it proves that the time-evolution of the one-particle reduced density matrix which is given by the many-body Schrödinger equation can approximately be described by a much simpler nonlinear one-particle equation.

To state the exact form of the one-particle equation, we specify the potentials $U$ we are interested in.

- For $\beta>0$, we consider the so called nonlinear Schrödinger (NLS) scaling $U(x)=$ $W_{\beta, N}(x)=N^{-1+2 \beta} W\left(N^{\beta} x\right)$, for a compactly supported, spherically symmetric and nonnegative potential $W \in L_{c}^{\infty}\left(\mathbb{R}^{2}, \mathbb{R}\right)$.

In the case of $\beta>1 / 2$, such a scaling models strong but short range repulsive interactions. The origin of the scaling can heuristically be motivated by the fact that for a completely factorized wave function $\Psi=\varphi^{\otimes N}$ with $\varphi \in H^{2}\left(\mathbb{R}^{2}, \mathbb{C}\right)$ the kinetic energy per particle ${ }^{2} \frac{1}{N}\left\langle\Psi, \sum_{k=1}^{N}\left(-\Delta_{k}\right) \Psi\right\rangle=-\langle\varphi, \Delta \varphi\rangle=\mathcal{O}(1)$ is of the same order as the potential energy per particle $\frac{1}{N}\left\langle\Psi, \sum_{1<j<k<N}^{N} W_{\beta}\left(x_{j}-x_{k}\right) \Psi\right\rangle=\mathcal{O}(1)$.

- We also consider exponentially scaled potentials $U(x)=V_{N}(x)=e^{2 N} V\left(e^{N} x\right)$ with $V \in L_{c}^{\infty}\left(\mathbb{R}^{2}, \mathbb{R}\right)$ being spherically symmetric and nonnegative. This scaling will be denoted Gross-Pitaevskii scaling in the following.

The motivation to consider an exponential scaling is similar to the Gross Pitaevskii scaling $V_{N}(x)=N^{2} V(N x)$ in three space dimensions. Namely, the kinetic and interaction energy are of the same order for a gas of fixed volume. This will be shown below, when discussing the scattering process of two particles, see (4). Furthermore, the interaction originates from a $N$-independent potential by rescaling space and time coordinates [see (7)]. Our results can be generalized to a wider class of $N$-dependent interactions covering most of the relevant cases discussed in the literature on two dimensional Bose gases [39].

For these scalings the condensate wave function $\varphi_{t}$ satisfies the cubic nonlinear Schrödinger equation

$$
\mathrm{i} \partial_{t} \varphi_{t}=\left(-\Delta+A_{t}\right) \varphi_{t}+b_{U}\left|\varphi_{t}\right|^{2} \varphi_{t}=: h_{b_{U}}^{G P} \varphi_{t}
$$

with initial datum $\varphi_{0}$. The precise definition of $b_{U}$ will be given in Definition 2.1. At the moment however if suffices to note that for the potentials from above we have $b_{W_{\beta, N}}=N\left\|W_{\beta, N}\right\|_{1}=\|W\|_{1}$ if $U=W_{\beta, N}$ and $b_{V_{N}}=4 \pi$ for $U=V_{N}$. In case that the coupling constant is given by $b_{V_{N}}=4 \pi$ Eq. (3) is also referred to as Gross-Pitaevskii equation.

We are going to explain on a heuristic level why the coupling constants differ in the NLS and Gross-Pitaevskii scaling. We first consider the exponential scaling and assume that the energy of the many-body state $\Psi_{t}$ is comparable to the ground state energy of the system. In this case, the wave function develops a short scale correlation structure which prevents the particles from being too close to each other [39]. If we neglect for the moment all but two particle correlations, one may heuristically think of $\Psi_{t}$ to be of Jastrow-type [38, p. 15 and p. 28], i.e. $\Psi_{t}\left(x_{1}, \ldots, x_{N}\right) \approx \prod_{i<j} F\left(x_{i}-x_{j}\right) \prod_{k=1}^{N} \varphi_{t}\left(x_{k}\right)$. The function $F$ accounts for pair correlations between the particles at scales of order $\mathcal{O}\left(e^{-N}\right)$. These correlations determine the time evolution of the condensate in a crucial

\footnotetext{
2 Throughout the paper we use the notation $\langle\cdot, \cdot\rangle=\langle\cdot, \cdot\rangle L_{S}^{2}\left(\mathbb{R}^{2 N}, \mathbb{C}\right)$ while $\langle\cdot, \cdot\rangle$ always refers to the scalar
} product of $L^{2}\left(\mathbb{R}^{2}, \mathbb{C}\right)$. 
manner and must therefore explicitly be taken into account. Since $V_{N}$ is a strong, short range potential, the interaction between the particles can in first order be described as a two-body scattering process. That is, the correlation function $F$ should approximately be given by the zero energy scattering state $j_{N, R} \in C^{1}\left(\mathbb{R}^{2}, \mathbb{R}\right)$ which is defined by

$$
\left\{\begin{array}{l}
\left(-\Delta_{x}+\frac{1}{2} e^{2 N} V\left(e^{N} x\right)\right) j_{N, R}(x)=0 \\
j_{N, R}(x)=1 \text { for }|x|=R
\end{array}\right.
$$

for some $R \in(0, \infty)$ used to normalize $j_{N, R}$ via the second line of (4). Note, that it is a peculiarity of two dimensional scattering states that $\lim _{x \rightarrow \infty}\left|j_{N, R}(x)\right|$ does not exist for short range potentials and can not be used for normalization. A particle at location $x$ then experiences the effective interaction

$$
\int_{\mathbb{R}^{2}} d^{2} y N V_{N}(x-y) j_{N, R}(x-y)\left|\varphi_{t}(y)\right|^{2} \approx\left|\varphi_{t}(x)\right|^{2} \int_{\mathbb{R}^{2}} d^{2} x N V_{N}(x) j_{N, R}(x),
$$

see e.g. [19] for a nice derivation. It will be shown in Sect. 5 that

$$
N \int_{\mathbb{R}^{2}} d^{2} x V_{N}(x) j_{N, R}(x)=N \frac{4 \pi}{\ln \left(\frac{R}{a e^{-N}}\right)},
$$

where $a$ denotes the scattering length of the potential $V$. Since $\frac{4 \pi}{\ln \left(\frac{R}{a e^{-N}}\right)} \approx \frac{4 \pi}{N}$ holds for $a>0$, the effective coupling $b_{V_{N}}$ will be given by $4 \pi$. This shows that the scaling we used gives us a system where the kinetic energy and the interaction energy are of the same order.

Let us now turn to the NLS scaling and consider for $\beta>0$ the scattering equation of the potential $W_{\beta, N}$

$$
\left\{\begin{array}{l}
\left(-\Delta_{x}+N^{-1+2 \beta} W\left(N^{\beta} x\right)\right) F_{N, \beta, R}(x)=0, \\
F_{N, \beta, R}(x)=1 \text { for }|x|=R
\end{array}\right.
$$

With $y=N^{\beta} x, \tilde{R}=N^{\beta} R$ and $G_{N, \beta, R}=F_{N, \beta, R}\left(N^{-\beta} \cdot\right)$, this can be written as

$$
\left\{\begin{array}{l}
\left(-\Delta_{y}+N^{-1} W(y)\right) G_{N, \beta, R}(y)=0, \\
G_{N, \beta, R}(y)=1 \text { for }|y|=\tilde{R}
\end{array}\right.
$$

Due to the factor $N^{-1}$, the zero energy scattering state is almost constant for large $N$, $F_{N, \beta, R}(x) \approx 1 \forall|x| \leq R$. It can therefore be concluded that the microscopic structure has a negligible effect on the effective interaction on each particle which is approximated by $^{3}$

$$
\begin{aligned}
& \int_{\mathbb{R}^{2}} d^{2} y N W_{\beta, N}(x-y) F_{N, \beta, R}(x-y)\left|\varphi_{t}(y)\right|^{2} \approx \int_{\mathbb{R}^{2}} d^{2} y N W_{\beta, N}(x-y)\left|\varphi_{t}(y)\right|^{2} \\
& \quad \rightarrow\|W\|_{1}\left|\varphi_{t}(x)\right|^{2} .
\end{aligned}
$$

\footnotetext{
${ }^{3}$ Even if the effect of the microscopic structure is negligible in the interaction, one should note that $\Psi_{t}$ is not close to a full product state $\prod_{k=1}^{N} \varphi_{t}\left(x_{k}\right)$ in norm. For certain types of interactions, it has rigorously been shown that $\Psi_{t}$ can be approximated by a quasifree state satisfying a Bogoliubov-type dynamics. We refer to $[5,12,23-25,31,35,43-46]$ for the precise statements.
} 
This yields to the correct coupling in the effective equation (3) in the case of $U(x)=$ $W_{\beta, N}(x)$.

Let us briefly compare the phenomenon of Bose-Einstein condensation in two and three dimensions. In three dimension the NLS scaling is defined by $N^{-1+3 \beta} W\left(N^{\beta} x\right)$ only for $0<\beta<1$ while in the case of $\beta=1$ the microscopic structure must be taken into account. This difference originates from the different form of the scattering state in two and three dimension, see Appendix $\mathrm{C}$ of [38]. In the case that the time evolution of $\Psi_{t}$ is generated by $H_{V_{N}}$ it is interesting to note that the effective evolution equation of $\varphi_{t}$ does not depend on the scattering length $a$. Also this contrasts the three-dimensional case, where the correct mean field coupling is given by $8 \pi a_{3 D}, a_{3 D}$ denoting the scattering length of the potential in three dimensions. The universal coupling $4 \pi$ in the case of a two-dimensional setup is known within the physical literature, see e.g. (30) and (A3) in [18] (note that $\hbar=1, m=\frac{1}{2}$ in our choice of coordinates).

Actually, our dynamical result complements a more general theory describing the ground state properties of dilute, two-dimensional Bose gases. It was shown in [39] that for a gas with repulsive interaction $V \geq 0$, the ground state energy per particle is to leading order given by either the Gross-Pitaevskii energy functional with coupling parameter $8 \pi /\left|\ln \left(\bar{\rho} a^{2}\right)\right|$ or a Thomas-Fermi type functional, depending on the diluteness of the gas, i.e. the mean-particle distance compared to the scattering length of the interaction. Here, $\bar{\rho}$ denotes the mean density of the gas and $a$ is the scattering length which must decrease exponentially with $N$ in the Gross-Pitaevskii limit [39, p. 20].

It should be pointed out that there has been some debate about the question whether two-dimensional Bose-Einstein condensation can be observed experimentally. This amounts to the question whether condensation takes place for temperatures $T>0$. For an ideal, noninteracting gas in a box, the standard grand canonical computation for the critical temperature $T_{c}$ of a Bose-Einstein condensate shows that there is no condensation for $T>0$. For trapped, noninteracting bosons in a confining power-law potential, the findings in [3] however show that in that case $T_{c}>0$ holds. Finally, it was proven in [37] that $\gamma_{\Psi}^{(1)}$ converges to $|\varphi\rangle\langle\varphi|$ in trace norm if $\Psi$ is the ground state of $H_{V_{N}}$ and $\varphi$ is the ground state of the Gross-Pitaevskii energy functional, see (8). It was furthermore proven that one does not observe $100 \%$ condensation in the ground state of an interacting homogenous system. The emergence of $100 \%$ Bose-Einstein condensation as a ground state phenomenon thus highly depends on the particular physical system. Our approach is the following: Initially, we assume the convergence of $\gamma_{\Psi_{0}}^{(1)}$ to $\left|\varphi_{0}\right\rangle\left\langle\varphi_{0}\right|$. We then show the persistence of condensation for time scales of order one. Our assumption is thus in agreement with the findings in [37].

The rigorous derivation of effective evolution equations is well known in the literature, see e.g. $[2,5,9-11,19-22,30,43,44,48-51]$ and references therein. For the twodimensional case we consider, it has been proven, for $0<\beta<3 / 4$ and $W$ nonnegative, that $\gamma_{\Psi_{t}}^{(1)}$ converges to $\left|\varphi_{t}\right\rangle\left\langle\varphi_{t}\right|$ as $N \rightarrow \infty$ [27]. For $0<\beta<1 / 6$, it has been established in [14] that the reduced density matrices converge, assuming that the potential $W$ is attractive, i.e. $W \leq 0$. This result was later extended to a larger class of scaling parameters $\beta$, under some assumptions on the negative part of the potential $W[26,34]$. In [45] a norm approximation to the two-dimensional focusing Schrödinger equation in the NLS scaling with $0<\beta<1$ was considered. Here, the evolution of the condensate is effectively described by the nonlinear Schrödinger equation while the evolution of the fluctuations around the condensate is governed by a quadratic Hamiltonian, resulting from Bogoliubov approximation. Another approach which relates more closely to the experimental setup is to consider a three-dimensional gas of bosons which is strongly 
confined in one spatial dimension. Then, one obtains an effective two-dimensional system in the unconfined directions. We remark that in this dimensional reduction two limits appear, the length scale in the confined direction and the scaling of the interaction in the unconfined directions. A derivation of the two-dimensional Gross-Pitaevskii equation from the three-dimensional quantum many-body dynamics of strongly confined bosons was just recently given in [7]. Further results in this direction can be found in [4, 6, 8, 15$17,28,29]$. For known results regarding the ground state properties of dilute Bose gases, we refer to the monograph [38], which also summarizes the papers $[37,39,40]$.

Our proof is based on [49], which covers the derivation of the time dependent GrossPitaevskii equation in three dimensions. In particular, the exponential scaling of the interaction forces us to adapt crucial ideas and refine many estimates. Additional difficulties arise amongst others from the logarithmic behaviour of the scattering state and the fact that $\left\|e^{2 N} V\left(e^{N} \cdot\right)\right\|_{L^{1}\left(\mathbb{R}^{2}, \mathbb{C}\right)} \sim 1$ while $\left\|N^{-1+3} V(N \cdot)\right\|_{L^{1}\left(\mathbb{R}^{3}, \mathbb{C}\right)} \sim N^{-1}$ in the three-dimensional Gross-Pitaevskii regime.

We shortly discuss the physical relevance of the Gross-Pitaevskii scaling. It is possible to rescale space- and time-coordinates in such a way that in the new coordinates the interaction is not $N$-dependent. Choosing $y=e^{N} x$ and $\tau=e^{2 N} t$ the Schrödinger equation reads

$$
\mathrm{i} \frac{d}{d \tau} \Psi_{e^{-2 N} \tau}=\left(-\sum_{j=1}^{N} \Delta_{y_{j}}+\sum_{1 \leq j<k \leq N} V\left(y_{j}-y_{k}\right)+\sum_{j=1}^{N} A_{e^{-2 N} \tau}\left(e^{-N} y_{j}\right)\right) \Psi_{e^{-2 N}} .
$$

The latter equation thus corresponds to an extremely dilute gas of bosons with density $\sim e^{-2 N}$. In order to observe a nontrivial dynamics, this condensate is then monitored over time scales of order $\tau \sim e^{2 N}$. Since the trapping potential is adjusted according to the density of the gas in the experiment, the $N$ dependence of $A_{e^{-2 N} \tau}\left(e^{-N} \cdot\right)$ is reasonable.

\section{Main Result}

Our main theorem consists of two parts, which consider potentials in the NLS and GrossPitaevskii scaling, respectively. For the proof of the theorem it is useful to enlarge the class of potentials in the NLS regime because it allows us in the derivation of the GrossPitaevskii equation to refer to various estimates that appear in first part of the proof.

Definition 2.1. (a) For $\beta>0$, we define the following space of sequences $\left(W_{\beta, N}\right)_{N \in \mathbb{N}}$.

$$
\begin{aligned}
\tilde{\mathcal{W}}_{\beta}= & \left\{\left(W_{\beta, N}\right)_{N \in \mathbb{N}} \mid W_{\beta, N} \in L_{c}^{\infty}\left(\mathbb{R}^{2}, \mathbb{R}\right), \exists C>0\right. \text { independent of } \\
& N \text { and } \beta: W_{\beta, N}(x) \geq 0 \forall x \in \mathbb{R}^{2}, \\
& \left\|W_{\beta, N}\right\|_{1} \leq C N^{-1},\left\|W_{\beta, N}\right\| \leq C N^{-1+\beta},\left\|W_{\beta, N}\right\|_{\infty} \leq C N^{-1+2 \beta}, \\
& \left.W_{\beta, N}(x)=0 \forall|x| \geq C N^{-\beta}, W_{\beta, N} \text { is spherically symmetric }\right\} .
\end{aligned}
$$

(b) For every $\left(W_{\beta, N}\right)_{N \in \mathbb{N}} \in \widetilde{\mathcal{W}}_{\beta}$ we define the coupling parameter $b_{W_{\beta}}=\lim _{N \rightarrow \infty} N \|$ $W_{\beta, N} \|_{1}$. 
(c) Define the set of potentials $\mathcal{W}_{\beta}$ by

$$
\begin{gathered}
\mathcal{W}_{\beta}=\left\{\left(W_{\beta, N}\right)_{N \in \mathbb{N}} \in \widetilde{\mathcal{W}}_{\beta} \mid \exists C>0 \text { independent of } N \text { and } \beta:\right. \\
\left.|N|\left|W_{\beta, N} \|_{1}-b_{W_{\beta}}\right| \leq C N^{-1} \ln (N)\right\} .
\end{gathered}
$$

To ease the notation, we often omit to display the dependence on $N$ and denote both the sequence $\left(W_{\beta, N}\right)_{N \in \mathbb{N}}$ and the element $W_{\beta, N}$ by $W_{\beta}$.

Remark 2.2. It should be noted that $N^{-1+2 \beta} W\left(N^{\beta} x\right) \in \mathcal{W}_{\beta}$, if $W \in L_{c}^{\infty}\left(\mathbb{R}^{2}, \mathbb{R}\right)$ is nonnegative and spherically symmetric. In this case, $b_{W_{\beta}}=\|W\|_{1}$.

For notational convenience, it is in addition helpful to define a class of potentials with Gross-Pitaevskii scaling.

Definition 2.3. Define the set of sequences of potentials $\left(V_{N}\right)_{N \in \mathbb{N}}$ as

$$
\begin{aligned}
\mathcal{V}_{N}= & \left\{\left(V_{N}\right)_{N \in \mathbb{N}} \mid \exists V \in L_{c}^{\infty}\left(\mathbb{R}^{2}, \mathbb{R}\right) \text { not being identically zero: } V_{N}(x)=e^{2 N} V\left(e^{N} x\right),\right. \\
& \left.V(x) \geq 0 \forall x \in \mathbb{R}^{2}, V \text { is spherically symmetric }\right\} .
\end{aligned}
$$

With a slight abuse of notation we use $V_{N}$ to denote the sequence $\left(V_{N}\right)_{N \in \mathbb{N}}$ and its $N$ th element.

For $U \in\left\{W_{\beta}, V_{N}\right\}$ and $A_{t} \in L^{\infty}\left(\mathbb{R}^{2}, \mathbb{R}\right)$, define the energy functional $\mathcal{E}_{U}: H^{1}\left(\mathbb{R}^{2 N}\right.$, $\mathbb{C}) \rightarrow \mathbb{R}$

$$
\mathcal{E}_{U}(\Psi)=N^{-1}\left\langle\Psi \Psi, H_{U} \Psi\right\rangle,
$$

where $\langle\langle\cdot, \cdot\rangle\rangle$ denotes the scalar product on $L^{2}\left(\mathbb{R}^{2 N}, \mathbb{C}\right)$. Furthermore, define the GrossPitaevskii energy functional $\mathcal{E}_{b_{U}}^{G P}: H^{1}\left(\mathbb{R}^{2}, \mathbb{C}\right) \rightarrow \mathbb{R}$

$$
\mathcal{E}_{b_{U}}^{G P}(\varphi)=\langle\nabla \varphi, \nabla \varphi\rangle+\left\langle\varphi,\left(A_{t}+\frac{1}{2} b_{U}|\varphi|^{2}\right) \varphi\right\rangle=\left\langle\varphi,\left(h_{b_{U}}^{G P}-\frac{1}{2} b_{U}|\varphi|^{2}\right) \varphi\right\rangle
$$

where $\langle\cdot, \cdot\rangle$ denotes the scalar product on $L^{2}\left(\mathbb{R}^{2}, \mathbb{C}\right)$. Note that both $\mathcal{E}_{U}(\Psi)$ and $\mathcal{E}_{b_{U}}^{G P}(\varphi)$ depend on $t$, due to the time varying external potential $A_{t}$. For the sake of readability, we will not indicate this time dependence explicitly. Our main theorem is the following.

Theorem 2.4. Let $\Psi_{0} \in L_{s}^{2}\left(\mathbb{R}^{2 N}, \mathbb{C}\right) \cap H^{2}\left(\mathbb{R}^{2 N}, \mathbb{C}\right)$ with $\left\|\Psi_{0}\right\|=1$. Let $\varphi_{0} \in L^{2}\left(\mathbb{R}^{2}, \mathbb{C}\right)$ with $\left\|\varphi_{0}\right\|=1$. Let the external potential $A_{t}$ satisfy $A . \in C^{1}\left(\mathbb{R}, L^{\infty}\left(\mathbb{R}^{2}, \mathbb{R}\right)\right)$.

(a) Let $\beta>0, W_{\beta} \in \mathcal{W}_{\beta}$ and let $\Psi_{t}$ the unique solution to $i \partial_{t} \Psi_{t}=H_{W_{\beta}} \Psi_{t}$ with initial datum $\Psi_{0}$. Let $\varphi_{t}$ the unique solution to $i \partial_{t} \varphi_{t}=h_{b_{W_{\beta}}}^{G P} \varphi_{t}$ with initial datum $\varphi_{0}$ and assume that $\varphi_{t} \in H^{3}\left(\mathbb{R}^{2}, \mathbb{C}\right) \forall t \in \mathbb{R}$. Let $\mathcal{E}_{W_{\beta}}\left(\Psi_{0}\right) \leq C$, where $C>0$ is a constant independent of $N$. Then, for any $t>0$ there exists a constant $0<C_{t}<\infty$, which 
depends on $t$ but not on $N$, such that

$$
\begin{aligned}
\left.\operatorname{Tr}\left|\gamma_{\Psi_{t}}^{(1)}-\right| \varphi_{t}\right\rangle\left\langle\varphi_{t}|| \leq\right. & e^{C_{t}}\left(\sqrt[4]{\left.\operatorname{Tr}\left|\gamma_{\Psi_{0}}^{(1)}-\right| \varphi_{0}\right\rangle\left\langle\varphi_{0}||\right.}\right. \\
& \left.+\sqrt{\left|\mathcal{E}_{W_{\beta}}\left(\Psi_{0}\right)-\mathcal{E}_{b_{W_{\beta}} P}^{G P}\left(\varphi_{0}\right)\right|}+N^{-\gamma} \sqrt{\ln (N)}\right) \\
\left|\mathcal{E}_{W_{\beta}}\left(\Psi_{t}\right)-\mathcal{E}_{b_{W_{\beta}}}^{G P}\left(\varphi_{t}\right)\right| \leq & e^{C_{t}}\left(\sqrt{\left.\operatorname{Tr}\left|\gamma_{\Psi_{0}}^{(1)}-\right| \varphi_{0}\right\rangle\left\langle\varphi_{0}||\right.}\right. \\
& \left.+\left|\mathcal{E}_{W_{\beta}}\left(\Psi_{0}\right)-\mathcal{E}_{b_{W_{\beta}} P}^{G P}\left(\varphi_{0}\right)\right|+N^{-2 \gamma} \ln (N)\right)
\end{aligned}
$$

where $\gamma=\beta$ for $0<\beta<1 / 12$ and $\gamma=1 / 20$ for $\beta \geq 1 / 12$.

(b) Let $V_{N} \in \mathcal{V}_{N}$ and let $\Psi_{t}$ the unique solution to $i \partial_{t} \Psi_{t}=H_{V_{N}} \Psi_{t}$ with initial datum $\Psi_{0}$. Let $\varphi_{t}$ the unique solution to $i \partial_{t} \varphi_{t}=h_{4 \pi}^{G P} \varphi_{t}$ with initial datum $\varphi_{0}$ and assume that $\varphi_{t} \in H^{3}\left(\mathbb{R}^{2}, \mathbb{C}\right) \forall t \in \mathbb{R}$. Let $\mathcal{E}_{V_{N}}\left(\Psi_{0}\right) \leq C$, where $C>0$ is a constant independent of $N$. Then, for any $t>0$ there exists a constant $0<C_{t}<\infty$, which depends on $t$ but not on $N$, such that

$$
\begin{aligned}
\left.\operatorname{Tr}\left|\gamma_{\Psi_{t}}^{(1)}-\right| \varphi_{t}\right\rangle\left\langle\varphi_{t}|| \leq\right. & e^{C_{t}}\left(\sqrt[4]{\left.\operatorname{Tr}\left|\gamma_{\Psi_{0}}^{(1)}-\right| \varphi_{0}\right\rangle\left\langle\varphi_{0}\right|} \mid\right. \\
& \left.+\sqrt{\left|\mathcal{E}_{V_{N}}\left(\Psi_{0}\right)-\mathcal{E}_{4 \pi}^{G P}\left(\varphi_{0}\right)\right|}+N^{-1 / 20}\right), \\
\left|\mathcal{E}_{V_{N}}\left(\Psi_{t}\right)-\mathcal{E}_{4 \pi}^{G P}\left(\varphi_{t}\right)\right| \leq & e^{C_{t}}\left(\sqrt{\left.\operatorname{Tr}\left|\gamma_{\Psi_{0}}^{(1)}-\right| \varphi_{0}\right\rangle\left\langle\varphi_{0}\right|} \mid\right. \\
& \left.+\left|\mathcal{E}_{V_{N}}\left(\Psi_{0}\right)-\mathcal{E}_{4 \pi}^{G P}\left(\varphi_{0}\right)\right|+N^{-1 / 10}\right) .
\end{aligned}
$$

Remarks. (a) If one considers initial many-body states which exhibit condensation and whose energy per particle converges to the corresponding Gross-Pitaevskii energy, i.e.

$$
\begin{array}{r}
\left.\lim _{N \rightarrow \infty} \operatorname{Tr}\left|\gamma_{\Psi_{0}}^{(1)}-\right| \varphi_{0}\right\rangle\left\langle\varphi_{0}||=0 \text { and } \lim _{N \rightarrow \infty}\left|\mathcal{E}_{U}\left(\Psi_{0}\right)-\mathcal{E}_{b_{U}}^{G P}\left(\varphi_{0}\right)\right|=0\right. \\
\text { with } U \in\left\{W_{\beta}, V_{N}\right\}
\end{array}
$$

it follows from Theorem 2.4 that

$\left.\lim _{N \rightarrow \infty} \operatorname{Tr}\left|\gamma_{\Psi_{t}}^{(1)}-\right| \varphi_{t}\right\rangle\left\langle\varphi_{t}||=0\right.$ and $\lim _{N \rightarrow \infty}\left|\mathcal{E}_{U}\left(\Psi_{t}\right)-\mathcal{E}_{b_{U}}^{G P}\left(\varphi_{t}\right)\right|=0$ for any $t>0$.

Our result consequently shows the stability of the condensate during the time evolution.

(b) It has been shown that in the limit $N \rightarrow \infty$ the energy-difference $\mathcal{E}_{V_{N}}\left(\Psi^{g s}\right)-$ $\mathcal{E}_{4 \pi}^{G P}\left(\varphi^{g s}\right) \rightarrow 0$, where $\Psi^{g s}$ is the ground state of a trapped Bose gas and $\varphi^{g s}$ the ground state of the respective Gross-Pitaevskii energy functional, see $[39,40]$. 
(c) The necessity to require $\varphi_{t} \in H^{3}\left(\mathbb{R}^{2}, \mathbb{C}\right)$ stems from the fact that the constant $C_{t}$ in (9) and (11) depends on $\left\|\varphi_{t}\right\|_{H^{3}}$, see the discussion before Lemma 4.7. For regular enough external potentials $A_{t}$ we expect the assumption $\varphi_{t} \in H^{3}\left(\mathbb{R}^{2}, \mathbb{C}\right)$ to follow from regularity assumptions on the initial datum $\varphi_{0}$. If $\varphi_{0} \in \Sigma^{3}\left(\mathbb{R}^{2}, \mathbb{C}\right)=$ $\left\{f \in L^{2}\left(\mathbb{R}^{2}, \mathbb{C}\right) \mid \sum_{\alpha+\beta<3}\left\|x^{\alpha} \partial_{x}^{\beta} f\right\|<\infty\right\}$ holds, the bound $\left\|\varphi_{t}\right\|_{H^{3}}<\infty$ has been proven for external potentials which are at most quadratic in space, see [13] and Lemma 4.7. In particular, for $\varphi_{0} \in \Sigma^{3}\left(\mathbb{R}^{2}, \mathbb{C}\right)$, the bound $\left\|\varphi_{t}\right\|_{H^{3}} \leq C$ with $C>0$ uniformly bounded in $t$ holds if the external potential is not present, i.e. $A_{t}=0$ [see [13] above (1.3.)].

(d) One can relax the conditions on the initial condition and only require $\Psi_{0} \in L_{s}^{2}\left(\mathbb{R}^{2 N}\right.$, $\mathbb{C})$ using a standard density argument.

\section{Organization of the Proof}

The method we are applying to prove Theorem 2.4 was originally introduced in [50] and later generalized to derive various mean-field equations [1,8,30,32,33,42,46-49]. Our proof is primarily based on [49] which covers the three-dimensional counterpart of our system. The key idea of the method is to show the existence of Bose-Einstein condensation not in terms of reduced density matrices but to consider an equivalent measure of condensation. Heuristically speaking, we count for each time $t$ the relative number of those particles which are not in the state of the condensate wave function $\varphi_{t}$. It is then possible to show that the rate of the particles which leave the condensate is small, if initially almost all particles were in the state $\varphi_{0}$. The counting of the particles will be performed with the help of a functional. In order to define it, we introduce the following operators.

Definition 3.1. Let $\varphi \in L^{2}\left(\mathbb{R}^{2}, \mathbb{C}\right)$ with $\|\varphi\|=1$.

(a) For any $1 \leq j \leq N$ the projectors $p_{j}^{\varphi}: L^{2}\left(\mathbb{R}^{2 N}, \mathbb{C}\right) \rightarrow L^{2}\left(\mathbb{R}^{2 N}, \mathbb{C}\right)$ and $q_{j}^{\varphi}$ : $L^{2}\left(\mathbb{R}^{2 N}, \mathbb{C}\right) \rightarrow L^{2}\left(\mathbb{R}^{2 N}, \mathbb{C}\right)$ are defined as

$$
p_{j}^{\varphi} \Psi=\varphi\left(x_{j}\right) \int \varphi^{*}\left(\tilde{x}_{j}\right) \Psi\left(x_{1}, \ldots, \tilde{x}_{j}, \ldots, x_{N}\right) d^{2} \tilde{x}_{j} \quad \forall \Psi \in L^{2}\left(\mathbb{R}^{2 N}, \mathbb{C}\right)
$$

and $q_{j}^{\varphi}=1-p_{j}^{\varphi}$. We shall also use, with a slight abuse of notation, the bra-ket notation $p_{j}^{\varphi}=\left|\varphi\left(x_{j}\right)\right\rangle\left\langle\varphi\left(x_{j}\right)\right|$.

(b) For any $0 \leq k \leq N$ we define the set

$$
\mathcal{S}_{k}=\left\{\vec{s}=\left(s_{1}, s_{2}, \ldots, s_{N}\right) \in\{0,1\}^{N} ; \sum_{j=1}^{N} s_{j}=k\right\}
$$

and the orthogonal projector $P_{k}^{\varphi}: L^{2}\left(\mathbb{R}^{2 N}, \mathbb{C}\right) \rightarrow L^{2}\left(\mathbb{R}^{2 N}, \mathbb{C}\right)$ as

$$
P_{k}^{\varphi}=\sum_{\vec{s} \in \mathcal{S}_{k}} \prod_{j=1}^{N}\left(p_{j}^{\varphi}\right)^{1-s_{j}}\left(q_{j}^{\varphi}\right)^{s_{j}} .
$$

For negative $k$ and $k>N$ we set $P_{k}^{\varphi}=0$. 
(c) For any function $m: \mathbb{N}_{0} \rightarrow \mathbb{R}_{0}^{+}$we define the operator $\widehat{m}^{\varphi}: L^{2}\left(\mathbb{R}^{2 N}, \mathbb{C}\right) \rightarrow L^{2}\left(\mathbb{R}^{2 N}, \mathbb{C}\right)$ as

$$
\widehat{m}^{\varphi}=\sum_{j=0}^{N} m(j) P_{j}^{\varphi} .
$$

We also need the shifted operators $\widehat{m}_{d}^{\varphi}: L^{2}\left(\mathbb{R}^{2 N}, \mathbb{C}\right) \rightarrow L^{2}\left(\mathbb{R}^{2 N}, \mathbb{C}\right)$ given by

$$
\widehat{m}_{d}^{\varphi}=\sum_{j=-d}^{N-d} m(j+d) P_{j}^{\varphi} \quad \text { with } d \in \mathbb{Z} .
$$

Following a general strategy ${ }^{4}$ we will define a functional $\alpha: L^{2}\left(\mathbb{R}^{2 N}, \mathbb{C}\right) \times L^{2}\left(\mathbb{R}^{2}, \mathbb{C}\right)$ $\rightarrow \mathbb{R}_{0}^{+}$such that

(a) $\alpha\left(\Psi_{0}, \varphi_{0}\right) \rightarrow 0$ for suitably chosen initial data $\left(\Psi_{0}, \varphi_{0}\right) \in L^{2}\left(\mathbb{R}^{2 N}, \mathbb{C}\right) \times L^{2}\left(\mathbb{R}^{2}, \mathbb{C}\right) .^{5}$

(b) If $\Psi_{t}$ is a solution of (2) and $\varphi_{t}$ a solution of (3), $\alpha\left(\Psi_{t}, \varphi_{t}\right)$ can be estimated by $\alpha\left(\Psi_{0}, \varphi_{0}\right)+\int_{0}^{t} d s C_{s}\left(\alpha\left(\Psi_{s}, \varphi_{s}\right)+\mathcal{O}(1)\right)$ for some time dependent constant $C_{s}$. Using a Grönwall type estimate, it then follows that $\alpha\left(\Psi_{t}, \varphi_{t}\right) \leq e^{2 \int_{0}^{t} d \tau C_{\tau}}\left(\alpha\left(\Psi_{0}, \varphi_{0}\right)+\right.$ $\mathcal{O}(1))$.

(c) $\alpha\left(\Psi_{t}, \varphi_{t}\right) \rightarrow 0$ implies the convergence of the one-particle reduced density matrix of $\Psi_{t}$ to $\left|\varphi_{t}\right\rangle\left\langle\varphi_{t}\right|$ in trace norm as well as the convergence of the energy per particle of the many-body system to the energy of the condensate wave function.

In [30,50] the mean field scaling $W_{0}(x)=N^{-1} W(x)$ and a condensate wave function which evolves according to the Hartree equation $i \partial_{t} \varphi_{t}=\left(-\Delta+A_{t}\right) \varphi_{t}+\left(W *\left|\varphi_{t}\right|^{2}\right) \varphi_{t}$ were considered in the three-dimensional setting. In these works it was shown that the persistence of condensation can be proven if one chooses

$$
\alpha\left(\Psi_{t}, \varphi_{t}\right)=\left\|\Psi_{t},\left(\widehat{n}^{\varphi_{t}}\right)^{j} \Psi_{t}\right\|,
$$

where $n(k)=\sqrt{k / N}, j>0$ and $\Psi_{t}$ is a solution of (2) with $U=W_{0}$. The choice $j=2$ corresponds to the functional $\left\langle\Psi_{t}, \sum_{k=0}^{N} \frac{k}{N} P_{k}^{\varphi_{t}} \Psi_{t}\right\rangle$, whose action on $\Psi_{t}$ can be viewed as "counting the relative number of particles which are not in the state $\varphi_{t}$ ". Other values of $j$ or a different choice of $\widehat{m}^{\varphi_{t}}$ should be understood as a weighted measure of counting the number of particles which are not in the condensate state. We will therefore sometimes call $m$ the weight function of the functional $\alpha$.

In this work we are interest in interaction potentials which get peaked as $N \rightarrow \infty$. As explained in Sect. 6.1, it is then no longer possible to obtain a Grönwall estimate with the previous choice of the functional and we have to adjust it in accordance with the scaling of the interaction. The precise definition of the functional and the proof of Theorem 2.4 are given in Sect. 6. In the preceding chapters we introduce the necessary preliminaries.

The rest of the paper is organized as follows:

(a) In Sect. 4 we start by fixing the notation. Afterwards, we recall important properties of the operator $\widehat{m}$ and explain the required regularity conditions on the solutions of the nonlinear Schrödinger equation.

\footnotetext{
4 For an extensive introduction to the method we refer to [50].

5 It should be noted that the requirement $\alpha\left(\Psi_{0}, \varphi_{0}\right) \rightarrow 0$ defines conditions on the initial states $\left(\Psi_{0}, \varphi_{0}\right)$.
} 
(b) In case of the exponential scaling, the interaction is so strong such that the many-body state develops a short scale correlation structure. This correlation structure affects the time evolution of the condensate and must therefore also be regarded in the definition of the functional. In Sect. 5, we explain the correlations structure in greater detail, provide certain estimates on the zero-energy scattering state and explain how the effective coupling parameter $b_{V_{N}}$ can be inferred from the microscopic structure.

(c) In Sect. 6 we prove Theorem 2.4. We first consider the potential $W_{\beta}$ and define a counting measure which allows us to establish a Grönwall estimate for all $\beta>0$. We will explain in detail how one arrives at this Grönwall estimate. Afterwards, the counting measure is adjusted to the case $V_{N}$, taking the microscopic structure $j_{N, R}$ of the wave function into account. We then establish a Grönwall estimate and finally prove the second part of the main theorem.

(d) In order to improve the readability of the paper we only state the estimates which are needed for the proof of Theorem 2.4 in Sect. 6. Their derivation is provided afterwards in Sect. 7.

\section{Preliminaries}

We will first fix the notation we are going to employ during the rest of the paper.

Notation 4.1. (a) Throughout the paper hats $\widehat{r}$ will always be used in the sense of Definition 3.1(c). The label $n$ will always be used for the function $n(k)=\sqrt{k / N}$.

(b) For better readability, we will often omit the upper index $\varphi$ on $p_{j}, q_{j}, P_{j}$ and $\widehat{\bullet}$ It will be placed exclusively in formulas where the $\varphi$-dependence is crucial.

(c) The operator norm, defined for any linear operator $f: L^{2}\left(\mathbb{R}^{2 N}, \mathbb{C}\right) \rightarrow L^{2}\left(\mathbb{R}^{2 N}, \mathbb{C}\right)$, will be denoted by

$$
\|f\|_{\text {op }}=\sup _{\psi \in L^{2}\left(\mathbb{R}^{2 N}, \mathbb{C}\right),\|\Psi\|=1}\|f \Psi\| .
$$

(d) We will bound expressions which are uniformly bounded in $N$ and $t$ by some constant $C$. Constants appearing in a sequence of estimates will not be distinguished, i.e. in $X \leq C Y \leq C Z$ the constants may differ.

(e) We will denote by $\mathcal{K}\left(\varphi_{t}, A_{t}\right)$ a generic polynomial with finite degree in $\left\|\varphi_{t}\right\|_{\infty},\left\|\nabla \varphi_{t}\right\|_{\infty},\left\|\nabla \varphi_{t}\right\|,\left\|\Delta \varphi_{t}\right\|,\left\|A_{t}\right\|_{\infty}, \int_{0}^{t} d s\left\|\dot{A}_{s}\right\|_{\infty}$ and $\left\|\dot{A}_{t}\right\|_{\infty}$. Note, in particular, that for a generic constant $C$ the inequality $C \leq \mathcal{K}\left(\varphi_{t}, A_{t}\right)$ holds. The exact form of $\mathcal{K}\left(\varphi_{t}, A_{t}\right)$ which appears in the final bounds can be reconstructed, collecting all contributions from the different estimates.

(f) We will denote for any multiplication operator $F: L^{2}\left(\mathbb{R}^{2}, \mathbb{C}\right) \rightarrow L^{2}\left(\mathbb{R}^{2}, \mathbb{C}\right)$ the corresponding operator

$$
\mathbb{1}^{\otimes(k-1)} \otimes F \otimes \mathbb{1}^{\otimes(N-k)}: L^{2}\left(\mathbb{R}^{2 N}, \mathbb{C}\right) \rightarrow L^{2}\left(\mathbb{R}^{2 N}, \mathbb{C}\right)
$$

acting on the $N$-particle Hilbert space by $F\left(x_{k}\right)$. In particular, we will use, for any $\Psi, \Omega \in L^{2}\left(\mathbb{R}^{2 N}, \mathbb{C}\right)$ the notation

$$
\left\langle\left\langle\Omega, \mathbb{1}^{\otimes(k-1)} \otimes F \otimes \mathbb{1}^{\otimes(N-k)} \Psi\right\rangle=\left\langle\left\langle\Omega, F\left(x_{k}\right) \Psi\right\rangle .\right.\right.
$$

In analogy, for any two-particle multiplication operator $K: L^{2}\left(\mathbb{R}^{2}, \mathbb{C}\right)^{\otimes 2} \rightarrow L^{2}$ $\left(\mathbb{R}^{2}, \mathbb{C}\right)^{\otimes 2}$, we denote the operator acting on any $\Psi \in L^{2}\left(\mathbb{R}^{2 N}, \mathbb{C}\right)$ by multiplication in the variable $x_{i}$ and $x_{j}$ by $K\left(x_{i}, x_{j}\right)$. In particular, we denote

$$
\left\langle\Omega, K\left(x_{i}, x_{j}\right) \Psi\right\rangle=\int_{\mathbb{R}^{2 N}} K\left(x_{i}, x_{j}\right) \Omega^{*}\left(x_{1}, \ldots, x_{N}\right) \Psi\left(x_{1}, \ldots, x_{N}\right) d^{2} x_{1} \ldots d^{2} x_{N} .
$$


Next, we prove some properties of the projectors $p_{j}$ and $q_{j}$, which are defined in Definition 3.1.

Lemma 4.2. (a) For any weights $m, r: \mathbb{N}_{0} \rightarrow \mathbb{R}_{0}^{+}$the commutation relations

$$
\widehat{m} \widehat{r}=\widehat{m r}=\widehat{r} \widehat{m}, \quad \widehat{m} p_{j}=p_{j} \widehat{m}, \quad \widehat{m} q_{j}=q_{j} \widehat{m}, \quad \widehat{m} P_{k}=P_{k} \widehat{m}
$$

hold.

(b) Let $n: \mathbb{N}_{0} \rightarrow \mathbb{R}_{0}^{+}$be given by $n(k)=\sqrt{k / N}$. Then, the square of $\widehat{n}$ equals the relative particle number operator of particles not in the state $\varphi$, i.e.

$$
(\widehat{n})^{2}=N^{-1} \sum_{j=1}^{N} q_{j}
$$

(c) For any weight $m: \mathbb{N}_{0} \rightarrow \mathbb{R}_{0}^{+}$and any function $f \in L^{\infty}\left(\mathbb{R}^{4}, \mathbb{C}\right)$ and any $j, k=$ $0,1,2$

$$
\widehat{m} Q_{j} f\left(x_{1}, x_{2}\right) Q_{k}=Q_{j} f\left(x_{1}, x_{2}\right) \widehat{m}_{j-k} Q_{k},
$$

where $Q_{0}=p_{1} p_{2}, Q_{1} \in\left\{p_{1} q_{2}, q_{1} p_{2}\right\}$ and $Q_{2}=q_{1} q_{2}$. Furthermore, for $j, k \in$ $\{0,1\}$ and $g \in L^{\infty}\left(\mathbb{R}^{2}, \mathbb{C}\right)$ the relations

$$
\widehat{m} \widetilde{Q}_{j} g\left(x_{1}\right) \widetilde{Q}_{k}=\widetilde{Q}_{j} g\left(x_{1}\right) \widehat{m}_{j-k} \widetilde{Q}_{k} \text { and } \widehat{m} \widetilde{Q}_{j} \nabla_{1} \widetilde{Q}_{k}=\widetilde{Q}_{j} \nabla_{1} \widehat{m}_{j-k} \widetilde{Q}_{k}
$$

hold, where $\widetilde{Q}_{0}=p_{1}$ and $\widetilde{Q}_{1}=q_{1}$.

(d) For any weight $m: \mathbb{N}_{0} \rightarrow \mathbb{R}_{0}^{+}$and any functions $f \in L^{\infty}\left(\mathbb{R}^{4}, \mathbb{C}\right), g \in L^{\infty}\left(\mathbb{R}^{2}, \mathbb{C}\right)$ the commutation relations

$$
\begin{aligned}
{\left[f\left(x_{1}, x_{2}\right), \widehat{m}\right] } & =\left[f\left(x_{1}, x_{2}\right), p_{1} p_{2}\left(\widehat{m}-\widehat{m}_{2}\right)+\left(p_{1} q_{2}+q_{1} p_{2}\right)\left(\widehat{m}-\widehat{m}_{1}\right)\right], \\
{\left[g\left(x_{1}\right), \widehat{m}\right] } & =q_{1} g\left(x_{1}\right)\left(\widehat{m}-\widehat{m}_{1}\right) p_{1}-p_{1}\left(\widehat{m}-\widehat{m}_{1}\right) g\left(x_{1}\right) q_{1}
\end{aligned}
$$

hold.

(e) Let $f \in L^{1}\left(\mathbb{R}^{2}, \mathbb{C}\right), g \in L^{2}\left(\mathbb{R}^{2}, \mathbb{C}\right)$. Then,

$$
\begin{aligned}
\left\|p_{j} f\left(x_{j}-x_{k}\right) p_{j}\right\|_{o p} & \leq\|f\|_{1}\|\varphi\|_{\infty}^{2}, \\
\left\|p_{j} g^{*}\left(x_{j}-x_{k}\right)\right\|_{\text {op }} & =\left\|g\left(x_{j}-x_{k}\right) p_{j}\right\|_{\text {op }} \leq\|g\|\|\varphi\|_{\infty}, \\
\|\left|\varphi\left(x_{j}\right)\right\rangle\left\langle\nabla_{j} \varphi\left(x_{j}\right)\right| g^{*}\left(x_{j}-x_{k}\right) \|_{o p} & =\left\|g\left(x_{j}-x_{k}\right) \nabla_{j} p_{j}\right\|_{o p} \leq\|g\|\|\nabla \varphi\|_{\infty} .
\end{aligned}
$$

Proof. (a) follows immediately from Definition 3.1, using that $p_{j}$ and $q_{j}$ are orthogonal projectors.

(b) Note that $\cup_{k=0}^{N} \mathcal{S}_{k}=\{0,1\}^{N}$, so $1=\sum_{k=0}^{N} P_{k}$. Using also $\left(q_{j}\right)^{2}=q_{j}$ and $q_{j} p_{j}=0$ we get

$$
\sum_{j=1}^{N} q_{j}=\sum_{j=1}^{N} q_{j} \sum_{k=0}^{N} P_{k}=\sum_{k=0}^{N} \sum_{j=1}^{N} q_{j} P_{k}=\sum_{k=0}^{N} k P_{k}=N \widehat{n^{2}}=N \widehat{n}^{2}
$$


(c) Using the definitions above we have

$$
\widehat{m} Q_{j} f\left(x_{1}, x_{2}\right) Q_{k}=\sum_{l=0}^{N} m(l) P_{l} Q_{j} f\left(x_{1}, x_{2}\right) Q_{k} .
$$

The number of projectors $q_{j}$ in $P_{l} Q_{j}$ in the coordinates $j=3, \ldots, N$ is equal to $l-j$. The $p_{j}$ and $q_{j}$ with $j=3, \ldots, N$ commute with $Q_{j} f\left(x_{1}, x_{2}\right) Q_{k}$. Thus $P_{l} Q_{j} f\left(x_{1}, x_{2}\right) Q_{k}=Q_{j} f\left(x_{1}, x_{2}\right) Q_{k} P_{l-j+k}$ and

$$
\begin{aligned}
\widehat{m} Q_{j} f\left(x_{1}, x_{2}\right) Q_{k} & =\sum_{l=0}^{N} m(l) Q_{j} f\left(x_{1}, x_{2}\right) Q_{k} P_{l-j+k} \\
& =\sum_{\widetilde{l}=k-j}^{N+k-j} Q_{j} f\left(x_{1}, x_{2}\right) m(\widetilde{l}+j-k) P_{l} Q_{k}=Q_{j} f\left(x_{1}, x_{2}\right) \widehat{m}_{j-k} Q_{k} .
\end{aligned}
$$

Similarly one gets the second and third formula.

(d) First note that

$$
\begin{aligned}
& {\left[f\left(x_{1}, x_{2}\right), \widehat{m}\right]-\left[f\left(x_{1}, x_{2}\right), p_{1} p_{2}\left(\widehat{m}-\widehat{m}_{2}\right)+p_{1} q_{2}\left(\widehat{m}-\widehat{m}_{1}\right)+q_{1} p_{2}\left(\widehat{m}-\widehat{m}_{1}\right)\right]} \\
& \quad=\left[f\left(x_{1}, x_{2}\right), q_{1} q_{2} \widehat{m}\right]+\left[f\left(x_{1}, x_{2}\right), p_{1} p_{2} \widehat{m}_{2}+p_{1} q_{2} \widehat{m}_{1}+q_{1} p_{2} \widehat{m}_{1}\right] .
\end{aligned}
$$

We will show that the right hand side is zero. Multiplying the right hand side with $p_{1} p_{2}$ from the left and using (c) one gets

$$
\begin{aligned}
& p_{1} p_{2} f\left(x_{1}, x_{2}\right) q_{1} q_{2} \widehat{m}+p_{1} p_{2} f\left(x_{1}, x_{2}\right) p_{1} p_{2} \widehat{m}_{2}-p_{1} p_{2} \widehat{m}_{2} f\left(x_{1}, x_{2}\right) \\
& \quad+p_{1} p_{2} f\left(x_{1}, x_{2}\right) p_{1} q_{2} \widehat{m}_{1}+p_{1} p_{2} f\left(x_{1}, x_{2}\right) q_{1} p_{2} \widehat{m}_{1} \\
& =p_{1} p_{2} \widehat{m}_{2} f\left(x_{1}, x_{2}\right) q_{1} q_{2}+p_{1} p_{2} \widehat{m}_{2} f\left(x_{1}, x_{2}\right) p_{1} p_{2}-p_{1} p_{2} \widehat{m}_{2} f\left(x_{1}, x_{2}\right) \\
& \quad+p_{1} p_{2} \widehat{m}_{2} f\left(x_{1}, x_{2}\right) p_{1} q_{2}+p_{1} p_{2} \widehat{m}_{2} f\left(x_{1}, x_{2}\right) q_{1} p_{2} \\
& =0 .
\end{aligned}
$$

Multiplying (18) with $p_{1} q_{2}$ from the left one gets

$$
\begin{aligned}
& p_{1} q_{2} f\left(x_{1}, x_{2}\right) q_{1} q_{2} \widehat{m}+p_{1} q_{2} f\left(x_{1}, x_{2}\right) p_{1} p_{2} \widehat{m}_{2}+p_{1} q_{2} f\left(x_{1}, x_{2}\right) p_{1} q_{2} \widehat{m}_{1} \\
& \quad+p_{1} q_{2} f\left(x_{1}, x_{2}\right) q_{1} p_{2} \widehat{m}_{1}-p_{1} q_{2} \widehat{m}_{1} f\left(x_{1}, x_{2}\right) .
\end{aligned}
$$

Using (c) the latter is zero. Also multiplying with $q_{1} p_{2}$ yields zero due to symmetry in interchanging $x_{1}$ with $x_{2}$. Multiplying (18) with $q_{1} q_{2}$ from the left one gets

$$
\begin{aligned}
& q_{1} q_{2} f\left(x_{1}, x_{2}\right) \widehat{m} q_{1} q_{2}-q_{1} q_{2} \widehat{m} f\left(x_{1}, x_{2}\right)+q_{1} q_{2} f\left(x_{1}, x_{2}\right) p_{1} p_{2} \widehat{m}_{2} \\
& \quad+q_{1} q_{2} f\left(x_{1}, x_{2}\right) p_{1} q_{2} \widehat{m}_{1}+q_{1} q_{2} f\left(x_{1}, x_{2}\right) q_{1} p_{2} \widehat{m}_{1}
\end{aligned}
$$

which is again zero and so is (18).

By means of the identity $1=p_{1}+q_{1}$ one has

$$
\begin{aligned}
{\left[g\left(x_{1}\right), \widehat{m}\right]=} & p_{1}\left(g\left(x_{1}\right) \widehat{m}-\widehat{m} g\left(x_{1}\right)\right) p_{1}+q_{1}\left(g\left(x_{1}\right) \widehat{m}-\widehat{m} g\left(x_{1}\right)\right) q_{1} \\
& +q_{1}\left(g\left(x_{1}\right) \widehat{m}-\widehat{m} g\left(x_{1}\right)\right) p_{1}+p_{1}\left(g\left(x_{1}\right) \widehat{m}-\widehat{m} g\left(x_{1}\right)\right) q_{1} .
\end{aligned}
$$

The second relation from part (d) then follows from (a) and (c). 
(e) To show (15), note that

$$
p_{j} f\left(x_{j}-x_{k}\right) p_{j}=p_{j}\left(f *|\varphi|^{2}\right)\left(x_{k}\right) .
$$

It follows that

$$
\left\|p_{j} f\left(x_{j}-x_{k}\right) p_{j}\right\|_{\text {op }} \leq\|f\|_{1}\|\varphi\|_{\infty}^{2} .
$$

For (16) we write

$$
\begin{aligned}
\left\|g\left(x_{j}-x_{k}\right) p_{j}\right\|_{\mathrm{op}}^{2} & =\sup _{\|\Psi\|=1}\left\|g\left(x_{j}-x_{k}\right) p_{j} \Psi\right\|^{2} \\
& =\sup _{\|\Psi\|=1}\left\langle\Psi \Psi, p_{j}\left|g\left(x_{j}-x_{k}\right)\right|^{2} p_{j} \Psi\right\rangle \\
& \leq\left\|p_{j}\left|g\left(x_{j}-x_{k}\right)\right|^{2} p_{j}\right\|_{\mathrm{op}} .
\end{aligned}
$$

With (15) we get (16). For (17) we use

$$
\begin{aligned}
\left\|g\left(x_{j}-x_{k}\right) \nabla_{j} p_{j}\right\|_{\mathrm{op}}^{2} & =\sup _{\|\Psi\|=1}\left\langle\Psi \Psi, p_{j}\left(|g|^{2} *|\nabla \varphi|^{2}\right)\left(x_{k}\right) \Psi\right\rangle \leq\left\||g|^{2} *|\nabla \varphi|^{2}\right\|_{\infty} \\
& \leq\|g\|^{2}\|\nabla \varphi\|_{\infty}^{2} .
\end{aligned}
$$

The Lemma then follows from the fact that, for bounded operators $A,\|A\|_{\text {op }}=$ $\left\|A^{*}\right\|_{\text {op }}$ holds, where $A^{*}$ is the adjoint operator of $A$.

Within our estimates we will encounter wave functions where some of the symmetry is broken (at this point the reader should exemplarily think of the wave function $V_{\beta}\left(x_{1}-\right.$ $\left.x_{2}\right) \Psi$ which is not symmetric under exchange of the variables $x_{1}$ and $x_{3}$ ). This leads to the following definition

Definition 4.3. For any finite set $\mathcal{M} \subset\{1,2, \ldots, N\}$, define the space $\mathcal{H}_{\mathcal{M}} \subset$ $L^{2}\left(\mathbb{R}^{2 N}, \mathbb{C}\right)$ as the set of functions which are symmetric in all variables in $\mathcal{M}$

$$
\Psi \in \mathcal{H}_{\mathcal{M}} \Leftrightarrow \Psi\left(x_{1}, \ldots, x_{j}, \ldots, x_{k}, \ldots, x_{N}\right)=\Psi\left(x_{1}, \ldots, x_{k}, \ldots, x_{j}, \ldots, x_{N}\right)
$$

for all $j, k \in \mathcal{M}$.

Based on the combinatorics of the $p_{j}$ and $q_{j}$, we obtain the following

Lemma 4.4. For any $f: \mathbb{N}_{0} \rightarrow \mathbb{R}_{0}^{+}$and any finite set $\mathcal{M}_{a} \subset\{1,2, \ldots, N\}$ with $1 \in \mathcal{M}_{a}$ and any finite set $\mathcal{M}_{b} \subset\{1,2, \ldots, N\}$ with $1,2 \in \mathcal{M}_{b}$

$$
\begin{aligned}
\left\|\widehat{f} q_{1} \Psi\right\|^{2} & \leq \frac{N}{\left|\mathcal{M}_{a}\right|}\|\widehat{f} \widehat{n} \Psi\|^{2} \quad \text { for any } \Psi \in \mathcal{H}_{\mathcal{M}_{a}}, \\
\left\|\widehat{f} q_{1} q_{2} \Psi\right\|^{2} & \leq \frac{N^{2}}{\left|\mathcal{M}_{b}\right|\left(\left|\mathcal{M}_{b}\right|-1\right)}\left\|\widehat{f}(\widehat{n})^{2} \Psi\right\|^{2} \quad \text { for any } \Psi \in \mathcal{H}_{\mathcal{M}_{b}} .
\end{aligned}
$$


Proof. Let $\Psi \in \mathcal{H}_{\mathcal{M}_{a}}$ for some finite set $1 \in \mathcal{M}_{a} \subset\{1,2, \ldots, N\}$. By Lemma 4.2 (b), (20) can be estimated as

$$
\begin{aligned}
\|\widehat{f} \widehat{n} \Psi\|^{2} & =\left\langle\Psi,(\widehat{f})^{2}(\widehat{n})^{2} \Psi\right\rangle=N^{-1} \sum_{k=1}^{N}\left\langle\left\langle\Psi,(\widehat{f})^{2} q_{k} \Psi\right\rangle\right. \\
& \geq N^{-1} \sum_{k \in \mathcal{M}_{a}}\left\langle\left\langle\Psi,(\widehat{f})^{2} q_{k} \Psi\right\rangle=\frac{\left|\mathcal{M}_{a}\right|}{N}\left\langle\Psi,(\widehat{f})^{2} q_{1} \Psi\right\rangle\right. \\
& =\frac{\left|\mathcal{M}_{a}\right|}{N}\left\|\widehat{f} q_{1} \Psi\right\|^{2} .
\end{aligned}
$$

Similarly, we obtain for $\Psi \in \mathcal{H}_{\mathcal{M}_{b}}$

$$
\begin{aligned}
\left\|\widehat{f}(\widehat{n})^{2} \Psi\right\|^{2} & =\left\langle\left\langle\Psi,(\widehat{f})^{2}(\widehat{n})^{4} \Psi\right\rangle \geq N^{-2} \sum_{j, k \in \mathcal{M}_{b}}\left\langle\Psi,(\widehat{f})^{2} q_{j} q_{k} \Psi\right\rangle\right. \\
& \left.=\frac{\left|\mathcal{M}_{b}\right|\left(\left|\mathcal{M}_{b}\right|-1\right)}{N^{2}}\left\langle\Psi,(\widehat{f})^{2} q_{1} q_{2} \Psi\right\rangle+\frac{\left|\mathcal{M}_{b}\right|}{N^{2}}\left\langle\Psi,(\widehat{f})^{2} q_{1} \Psi\right\rangle\right\rangle \\
& \geq \frac{\left|\mathcal{M}_{b}\right|\left(\left|\mathcal{M}_{b}\right|-1\right)}{N^{2}}\left\|\widehat{f} q_{1} q_{2} \Psi\right\|^{2}
\end{aligned}
$$

which concludes the Lemma.

Corollary 4.5. Let $\Psi \in L_{s}^{2}\left(\mathbb{R}^{2 N}, \mathbb{C}\right)$. For any weight $m: \mathbb{N}_{0} \rightarrow \mathbb{R}_{0}^{+}$

$$
\begin{aligned}
\left\|\nabla_{2} \widehat{m} q_{2} \Psi\right\| & \leq 2\|\widehat{m}\|_{o p}\left\|\nabla_{2} q_{2} \Psi\right\|, \\
\left\|\nabla_{2} \widehat{m} q_{1} q_{2} \Psi\right\| & \leq C\|\widehat{m} \hat{n}\|_{o p}\left\|\nabla_{2} q_{2} \Psi\right\| .
\end{aligned}
$$

Proof. Using $p_{2}+q_{2}=1$ and triangle inequality,

$$
\begin{aligned}
\left\|\nabla_{2} \widehat{m} q_{2} \Psi\right\| & \leq\left\|p_{2} \nabla_{2} \widehat{m} q_{2} \Psi\right\|+\left\|q_{2} \nabla_{2} \widehat{m} q_{2} \Psi\right\|, \\
\left\|\nabla_{2} \widehat{m} q_{1} q_{2} \Psi\right\| & \leq\left\|p_{2} \nabla_{2} \widehat{m} q_{1} q_{2} \Psi\right\|+\left\|q_{2} \nabla_{2} \widehat{m} q_{1} q_{2} \Psi\right\| .
\end{aligned}
$$

With Lemma 4.2 (c) we get

$$
\text { (24) }=\left\|\widehat{m}_{1} p_{2} \nabla_{2} q_{2} \Psi\right\|+\left\|\widehat{m} q_{2} \nabla_{2} q_{2} \Psi\right\| \leq\left(\left\|\widehat{m}_{1}\right\|_{\text {op }}+\|\widehat{m}\|_{\text {op }}\right)\left\|\nabla_{2} q_{2} \Psi\right\| .
$$

Note that the wave function $p_{2} \nabla_{2} q_{2} \Psi$ is symmetric under the exchange of any two variables but $x_{2}$. Thus we can use Lemma 4.4 to get

$$
\begin{aligned}
(25) & =\left\|q_{1} \widehat{m}_{1} p_{2} \nabla_{2} q_{2} \Psi\right\|+\left\|q_{1} \widehat{m} q_{2} \nabla_{2} q_{2} \Psi\right\| \\
& \leq \frac{N}{N-1}\left(\left\|\widehat{m}_{1} \widehat{n}\right\|_{\text {op }}+\|\widehat{m} \widehat{n}\|_{\text {op }}\right)\left\|\nabla_{2} q_{2} \Psi\right\| .
\end{aligned}
$$

Since $\sqrt{k} \leq \sqrt{k+1}$ for $k \geq 0$ it follows that the latter is bounded by

$$
C\left(\left\|\widehat{m}_{1} \widehat{n}_{1}\right\|_{\mathrm{op}}+\|\widehat{m} \widehat{n}\|_{\mathrm{op}}\right)\left\|\nabla_{2} q_{2} \Psi\right\| \text {. }
$$

Using that $\|\widehat{r}\|_{\text {op }}=\sup _{0 \leq k \leq N}\{r(k)\}=\left\|\widehat{r}_{d}\right\|_{\text {op }}$ for any $d \in \mathbb{N}$ and any weight $r$, the Corollary follows. 
Lemma 4.6. Let $\Omega, \chi \in \mathcal{H}_{\mathcal{M}}$ for some $\mathcal{M}$, let $1 \notin \mathcal{M}$ and $2,3 \in \mathcal{M}$. Let $O_{j, k}$ be an operator acting on the $j^{\text {th }}$ and $k^{\text {th }}$ coordinate. Then

$$
\left|\left\langle\Omega, O_{1,2} \chi\right\rangle\right| \leq\|\Omega\|^{2}+\left|\left\langle O_{1,2} \chi, O_{1,3} \chi\right\rangle\right|+(|\mathcal{M}|)^{-1}\left\|O_{1,2} \chi\right\|^{2} .
$$

Proof. Using symmetry and Cauchy Schwarz

$$
\left|\left\langle\Omega, O_{1,2} \chi\right\rangle\right|=|\mathcal{M}|^{-1}\left|\left\langle\Omega, \sum_{j \in \mathcal{M}} O_{1, j} \chi\right\rangle\right| \leq|\mathcal{M}|^{-1}\|\Omega\|\left\|\sum_{j \in \mathcal{M}} O_{1, j} \chi\right\| .
$$

For the second factor we can write

$$
\begin{aligned}
\left\|\sum_{j \in \mathcal{M}} O_{1, j} \chi\right\|^{2} & \left.=\left\langle\sum_{j \in \mathcal{M}} O_{1, j} \chi, \sum_{k \in \mathcal{M}} O_{1, k} \chi\right\rangle\right\rangle \\
& \leq \sum_{j \in \mathcal{M}}\left|\left\langle O_{1, j} \chi, O_{1, j} \chi\right\rangle\right|+\mid \sum_{j \neq k \in \mathcal{M}}\left\langle\left\langle O_{1, j} \chi, O_{1, k} \chi\right\rangle\right| \\
& \leq|\mathcal{M}|\left|\left\langle O_{1,2} \chi, O_{1,2} \chi\right\rangle\right|+|\mathcal{M}|(|\mathcal{M}|-1)\left|\left\langle O_{1,2} \chi, O_{1,3} \chi\right\rangle\right| .
\end{aligned}
$$

Since $a b \leq 1 / 2 a^{2}+1 / 2 b^{2}$ and $(a+b)^{2} \leq 2 a^{2}+2 b^{2}$ holds for any real numbers $a$ and $b$, the Lemma follows.

In our estimates, we need the regularity conditions

$$
\left\|\nabla \varphi_{t}\right\|_{\infty}<\infty, \quad\left\|\varphi_{t}\right\|_{\infty}<\infty, \quad\left\|\nabla \varphi_{t}\right\|<\infty, \quad\left\|\Delta \varphi_{t}\right\|<\infty .
$$

That is, we need $\varphi_{t} \in H^{2}\left(\mathbb{R}^{2}, \mathbb{C}\right) \cap W^{1, \infty}\left(\mathbb{R}^{2}, \mathbb{C}\right)$. Then, $\left\|\Delta\left|\varphi_{t}\right|^{2}\right\|,\left\|\Delta\left|\varphi_{t}\right|^{2}\right\|_{1}$ and $\left\|\varphi_{t}^{2}\right\|$, which also appear in our estimates, can be bounded by

$$
\begin{aligned}
\Delta\left|\varphi_{t}\right|^{2} & =\varphi_{t}^{*} \Delta \varphi_{t}+\varphi_{t} \Delta \varphi_{t}^{*}+2\left(\nabla \varphi_{t}^{*}\right) \cdot\left(\nabla \varphi_{t}\right) \\
\left\|\Delta\left|\varphi_{t}\right|^{2}\right\| & \leq 2\left\|\Delta \varphi_{t}\right\|\left\|\varphi_{t}\right\|_{\infty}+2\left\|\nabla \varphi_{t}\right\|\left\|\nabla \varphi_{t}\right\|_{\infty} \\
\left\|\Delta\left|\varphi_{t}\right|^{2}\right\|_{1} & \leq 4\left\|\Delta \varphi_{t}\right\| \\
\left\|\varphi_{t}^{2}\right\| & \leq\left\|\varphi_{t}\right\|_{\infty}\left\|\varphi_{t}\right\| .
\end{aligned}
$$

Recall the Sobolev embedding Theorem, which implies in particular $H^{k}\left(\mathbb{R}^{2}, \mathbb{C}\right)=$ $W^{k, 2}\left(\mathbb{R}^{2}, \mathbb{C}\right) \subset C^{k-2}\left(\mathbb{R}^{2}, \mathbb{C}\right)$. If $\varphi \in C^{1}\left(\mathbb{R}^{2}, \mathbb{C}\right) \cap H^{1}\left(\mathbb{R}^{2}, \mathbb{C}\right)$, then $\varphi \in W^{1, \infty}\left(\mathbb{R}^{2}, \mathbb{C}\right)$ follows since both $\varphi$ and $\nabla \varphi$ have to decay at infinity. Thus, $\varphi_{t} \in H^{3}\left(\mathbb{R}^{2}, \mathbb{C}\right)$ implies $\varphi_{t} \in H^{2}\left(\mathbb{R}^{2}, \mathbb{C}\right) \cap W^{1, \infty}\left(\mathbb{R}^{2}, \mathbb{C}\right)$, which suffices for our estimates. Since $\varphi_{t}$ obeys a defocusing nonlinear Schrödinger equation, we expect the regularity of the solution $\varphi_{t}$ to follow from the regularity of the initial datum $\varphi_{0}$. For a certain class of external potentials $A_{t}$ this has been proven in [13]:

Lemma 4.7. Let $\varphi_{0} \in \Sigma^{k}\left(\mathbb{R}^{2}, \mathbb{C}\right)=\left\{f \in L^{2}\left(\mathbb{R}^{2}, \mathbb{C}\right) \mid \sum_{\alpha+\beta \leq k}\left\|x^{\alpha} \partial_{x}^{\beta} f\right\|<\infty\right\}$, for $k \geq 2$. Let, for $b>0, \varphi_{t}$ be the unique solution to

$$
i \partial_{t} \varphi_{t}=\left(-\Delta+A_{t}+b\left|\varphi_{t}\right|^{2}\right) \varphi_{t} .
$$

Let $A . \in L_{\text {loc }}^{\infty}\left(\mathbb{R}_{t} \times \mathbb{R}_{x}^{2}, \mathbb{C}\right)$ real valued and smooth with respect to the space variable: for (almost) all $t \in \mathbb{R}$, the map $x \mapsto A_{t}(x)$ is $C^{\infty}$. Moreover, $A_{t}$ is at most quadratic in space, uniformly w.r.t. time $t$ :

$$
\forall \alpha \in \mathbb{N}^{2},|\alpha| \geq 2, \quad \partial_{x}^{\alpha} A . \in L^{\infty}\left(\mathbb{R}_{t} \times \mathbb{R}_{x}^{d}, \mathbb{C}\right) .
$$

In addition, $t \mapsto \sup _{|x| \leq 1}\left|A_{t}(x)\right|$ belongs to $L^{\infty}(\mathbb{R}, \mathbb{C})$. Then 
(a) $\varphi_{t} \in \Sigma^{k}\left(\mathbb{R}^{2}, \mathbb{C}\right)$, which implies $\varphi_{t} \in H^{k}\left(\mathbb{R}^{2}, \mathbb{C}\right)$.

(b) $\left\|\varphi_{t}\right\|=\left\|\varphi_{0}\right\|$.

(c) Let $\varphi_{0} \in \Sigma^{3}\left(\mathbb{R}^{2}, \mathbb{C}\right)$. Assume in addition that $A . \in C^{1}\left(\mathbb{R}, L^{\infty}\left(\mathbb{R}^{2}, \mathbb{R}\right)\right)$. Then, for any fixed $t \geq 0, \mathcal{K}\left(\varphi_{t}, A_{t}\right)<\infty$ follows.

Proof. Part (a) is Corollary 1.4. in [13]. We like to remark that $\left\|\varphi_{t}\right\|_{H^{k}} \leq C$ holds, if $A_{t}=0$, see Section 1.2. in [13]. The conditions on $A_{t}$ are for example satisfied if $A_{t} \in C_{c}^{\infty}\left(\mathbb{R}^{2}, \mathbb{R}\right)$ for all $t \in \mathbb{R}, A_{t}(x)=0$, for all $|t| \geq T$. Part (b) can be verified directly, using the existence of global in time solutions. Part (c) follows from (a) and the embedding $H^{3}\left(\mathbb{R}^{2}, \mathbb{C}\right) \subset H^{2}\left(\mathbb{R}^{2}, \mathbb{C}\right) \cap W^{1, \infty}\left(\mathbb{R}^{2}, \mathbb{C}\right)$.

\section{Microscopic Structure in 2 Dimensions}

5.1. The scattering state. In this section we analyze the microscopic structure which is induced by $V_{N}$. In particular, we explain why the dynamical properties of the system are determined by the low energy scattering regime.

Definition 5.1. Let $V_{N} \in \mathcal{V}_{N}$. For any $R \geq \operatorname{diam}\left(\operatorname{supp}\left(V_{N}\right)\right)$, we define the zero energy scattering state $j_{N, R} \in C^{1}\left(\mathbb{R}^{2}, \mathbb{R}\right)$ by

$$
\left\{\begin{array}{l}
\left(-\Delta_{x}+\frac{1}{2} e^{2 N} V\left(e^{N} x\right)\right) j_{N, R}(x)=0 \\
j_{N, R}(x)=1 \text { for }|x|=R
\end{array}\right.
$$

Next, we want to recall some important properties of the scattering state $j_{N, R}$, see also Appendix C of [38].

Lemma 5.2. Let $V_{N} \in \mathcal{V}_{N}$. Define $I_{R}=\int_{\mathbb{R}^{2}} d^{2} x V_{N}(x) j_{N, R}(x)$. For the scattering state defined previously the following relations hold:

(a) There exists a nonnegative number a, called scattering length of the potential $V$, such that

$$
I_{R}=\frac{4 \pi}{\ln \left(\frac{e^{N} R}{a}\right)}
$$

(in the case $a=0$ we have $I_{R}=0$ ). The scattering length a does not depend on $R$ and fulfills $a \leq \operatorname{diam}(\operatorname{supp}(V))$. Furthermore, $I_{R} \geq 0$ holds.

(b) $j_{N, R}$ is a nonnegative function which is spherically symmetric in $|x|$. For $|x| \geq$ $\operatorname{diam}\left(\operatorname{supp}\left(V_{N}\right)\right), j_{N, R}$ is given by

$$
j_{N, R}(x)=1+\frac{1}{\ln \left(\frac{e^{N} R}{a}\right)} \ln \left(\frac{|x|}{R}\right) .
$$

Proof. (a)+(b) Rescaling $x \rightarrow e^{N} x=y$, we obtain, setting $\tilde{R}=e^{N} R$ and $s_{\tilde{R}}(y)=$ $j_{0, e^{N} R}(y)$, the unscaled scattering equation

$$
\left\{\begin{array}{l}
\left(-\Delta_{y}+\frac{1}{2} V(y)\right) s_{\tilde{R}}(y)=0 \\
s_{\tilde{R}}(y)=1 \text { for }|y|=\tilde{R}
\end{array}\right.
$$


Since we assume $V$ to be nonnegative, one can define the scattering state $s_{\tilde{R}}$ by a variational principle. Theorem C.1 in [38] then implies that $s_{\tilde{R}}$ is a nonnegative, spherically symmetric function in $|y|$. It is then easy to verify that for diam $(\operatorname{supp}(V)) \leq|y|$ there exists a number $A \in \mathbb{R}$ such that

$$
s_{\tilde{R}}(y)=1+\frac{A}{4 \pi} \ln \left(\frac{|y|}{\tilde{R}}\right) .
$$

Next, we show that $A=\int_{\mathbb{R}^{2}} d^{2} y V(y) s_{\tilde{R}}(y)$. This can be seen by noting that, for $r>\operatorname{diam}(\operatorname{supp}(V))$,

$$
\begin{aligned}
\int_{\mathbb{R}^{2}} d^{2} y V(y) s_{\tilde{R}}(y) & =2 \int_{B_{r}(0)} d^{2} y \Delta s_{\tilde{R}}(y)=2 \int_{\partial B_{r}(0)} \nabla s_{\tilde{R}}(y) \cdot d s \\
& =\frac{A}{2 \pi} \int_{\partial B_{r}(0)} \nabla \ln (|y|) \cdot d s=\frac{A}{2 \pi} \int_{0}^{2 \pi} \frac{1}{r} r d \varphi \\
& =A .
\end{aligned}
$$

By Theorem C. 1 in [38], there exists a number $a \geq 0$, not depending on $\tilde{R}$, such that for all $|y| \geq \operatorname{diam}(\operatorname{supp}(V))$

$$
s_{\tilde{R}}(y)=\frac{\ln (|y| / a)}{\ln (\tilde{R} / a)} .
$$

Comparing this with (28), we obtain

$$
\int_{\mathbb{R}^{2}} V(y) s_{\tilde{R}}(y) d y^{2}=\frac{4 \pi}{\ln \left(\frac{\tilde{R}}{a}\right)} .
$$

Since $s_{\tilde{R}}$ is nonnegative, it furthermore follows that $a \leq \operatorname{diam}(\operatorname{supp}(V))$. This directly implies $A \geq 0$. By scaling, we obtain

$$
I_{R}=\int_{\mathbb{R}^{2}} V_{N}(y) j_{N, R}(y) d y^{2}=\int_{\mathbb{R}^{2}} V(y) s_{\tilde{R}}(y) d y^{2}=\frac{4 \pi}{\ln \left(\frac{e^{N} R}{a}\right)} .
$$

Assuming that the energy per particle $\mathcal{E}_{V_{N}}(\Psi)$ is of order one, the wave function $\Psi$ will have a microscopic structure near the interactions $V_{N}$, given by $j_{N, R}$. The interaction among two particles is then determined by $\frac{4 \pi}{N+\ln \left(\frac{R}{a}\right)} \approx \frac{4 \pi}{N}$. Keeping in mind that each particle interacts with all other $N-1$ particles, we obtain the effective Gross-Pitaevskii equation, for $\varphi_{t} \in H^{2}\left(\mathbb{R}^{2}, \mathbb{C}\right)$

$$
i \partial_{t} \varphi_{t}(x)=\left(-\Delta+A_{t}+4 \pi\left|\varphi_{t}(x)\right|^{2}\right) \varphi_{t}(x) .
$$

Thus, choosing $V_{N}(x)=e^{2 N} V\left(e^{N} x\right)$ leads in our setting to an effective one-particle equation which is determined by the low energy scattering behavior of the particles. We remark that, for any $s>0$, the potential $e^{2 N s} V\left(e^{N s} x\right)$ yields to the coupling $4 \pi / s$. 
5.2. Properties of the scattering state. Note that the potential $V_{N}$ is strongly peaked within an exponentially small region. In order to control the short scale structure of $\Psi_{t}$, we define a potential $M_{\mu}$ with softer scaling behaviour in such a way that the potential $V_{N}-M_{\mu}$ has scattering length zero. This allows us to "replace" $V_{N}$ by $M_{\mu}$, which has better scaling behavior and is easier to control. In particular, $\left\|M_{\mu}\right\| \leq C N^{-1+\mu}$ can be controlled for $\mu$ sufficiently small.

Definition 5.3. Let $V_{N} \in \mathcal{V}_{N}$. For any $\mu>0$ and any $R_{\mu} \geq N^{-\mu}$ we define the potential $M_{\mu}$ via

$$
M_{\mu}(x)= \begin{cases}4 \pi N^{-1+2 \mu} & \text { if } N^{-\mu}<|x| \leq R_{\mu} \\ 0 & \text { else }\end{cases}
$$

Furthermore, we define the zero energy scattering state $f_{\mu} \in C^{1}\left(\mathbb{R}^{2}, \mathbb{R}\right)$ of the potential $\frac{1}{2}\left(V_{N}-M_{\mu}\right)$, that is

$$
\left\{\begin{array}{l}
\left(-\Delta_{x}+\frac{1}{2}\left(V_{N}(x)-M_{\mu}(x)\right)\right) f_{\mu}(x)=0, \\
f_{\mu}(x)=1 \text { for }|x|=R_{\mu} .
\end{array}\right.
$$

Note that $M_{\mu}$ and $f_{\mu}$ depend on $R_{\mu}$.

Remark 5.4. In the following, we choose $R_{\mu}$ to be the smallest value such that the scattering length of the potential $\left(V_{N}-M_{\mu}\right)$ is zero which is equivalent to the condition $\int_{\mathbb{R}} d^{2} x\left(V_{N}(x)-M_{\mu}(x)\right) f_{\mu}(x)=0$. The existence of such $R_{\mu}<\infty$ will be proven in Lemma 5.5.

Note, that choosing $R_{\mu}$ to be the minimal value such that $\left(V_{N}-M_{\mu}\right)$ has scattering length zero excludes the possibility for bound states for the potential. This will be shown in Lemma 7.10 (a). Heuristically speaking, the absence of bound states can be seen in the following way: The attractive part of the potential, i.e. $-M_{\mu}$, is chosen to be as small as possible, i.e. just to compensates the repulsive part. Then, there is not enough attractiveness left to form a bound state.

Lemma 5.5. For the scattering state $f_{\mu}$, defined by (30), the following relations hold:

(a) There exists a minimal value $R_{\mu}<\infty$ such that $\int_{\mathbb{R}^{2}} d^{2} x\left(V_{N}(x)-M_{\mu}(x)\right) f_{\mu}(x)=0$.

For the rest of the paper we assume that $R_{\mu}$ is the minimum we get in $(a)$.

(b) There exists $K_{\mu} \in \mathbb{R}, K_{\mu}>0$ such that $K_{\mu} f_{\mu}(x)=j_{N, R_{\mu}}(x) \forall|x| \leq N^{-\mu}$.

(c) For $N$ sufficiently large the supports of $V_{N}$ and $M_{\mu}$ do not overlap.

(d) $f_{\mu}$ is a positive, monotone nondecreasing function in $|x|$.

(e)

$$
f_{\mu}(x)=1 \text { for }|x| \geq R_{\mu} .
$$

(f)

$$
1 \geq K_{\mu} \geq 1+\frac{1}{N+\ln \left(\frac{R_{\mu}}{a}\right)} \ln \left(\frac{N^{-\mu}}{R_{\mu}}\right) .
$$

(g) $R_{\mu} \leq C N^{-\mu}$. 
For any fixed $0<\mu, N$ sufficiently large such that $V_{N}$ and $M_{\mu}$ do not overlap, we obtain (h)

$$
\left|N\left\|V_{N} f_{\mu}\right\|_{1}-4 \pi\right|=\left|N\left\|M_{\mu} f_{\mu}\right\|_{1}-4 \pi\right| \leq C \frac{\ln (N)}{N}
$$

(i) Define

$$
g_{\mu}(x)=1-f_{\mu}(x)
$$

Then,

$$
\left\|g_{\mu}\right\|_{1} \leq C N^{-1-2 \mu} \ln (N), \quad\left\|g_{\mu}\right\| \leq C N^{-1-\mu} \ln (N), \quad\left\|g_{\mu}\right\|_{\infty} \leq 1 .
$$

(j)

$$
\left|N\left\|M_{\mu}\right\|_{1}-4 \pi\right| \leq C \frac{\ln (N)}{N} .
$$

(k)

$$
M_{\mu} \in \mathcal{W}_{\mu}, M_{\mu} f_{\mu} \in \mathcal{W}_{\mu}
$$

Proof. (a) In the following, we will sometimes denote, with a slight abuse of notation, $f_{\mu}(x)=f_{\mu}(r)$ and $j_{N, R}(x)=j_{N, R}(r)$ for $r=|x|$ (for this, recall that $f_{\mu}$ and $j_{N, R}$ are radially symmetric). We further denote by $f_{\mu}^{\prime}(r)$ the derivative of $f_{\mu}$ with respect to $r$.

We first show by contradiction that there exists a $x_{0} \in \mathbb{R}^{2}, \quad\left|x_{0}\right| \leq N^{-\mu}$, such that $f_{\mu}\left(x_{0}\right) \neq 0$. For this, assume that $f_{\mu}(x)=0$ for all $|x| \leq N^{-\mu}$. Since $f_{\mu}$ is continuous, there exists a maximal value $r_{0} \geq N^{-\mu}$ such that the scattering equation (30) is equivalent to

$$
\left\{\begin{array}{l}
\left(-\Delta_{x}-\frac{1}{2} M_{\mu}(x)\right) f_{\mu}(x)=0, \\
f_{\mu}(x)=1 \text { for }|x|=R_{\mu}, \\
f_{\mu}(x)=0 \text { for }|x| \leq r_{0} .
\end{array}\right.
$$

Using (30) and Gauss'-theorem, we further obtain

$$
f_{\mu}^{\prime}(r)=\frac{1}{4 \pi r} \int_{B_{r}(0)} d^{2} x\left(V_{N}(x)-M_{\mu}(x)\right) f_{\mu}(x) .
$$

(33) and (34) then imply for $r>r_{0}$

$$
\begin{aligned}
\left|f_{\mu}^{\prime}(r)\right| & =\frac{1}{4 \pi r}\left|\int_{B_{r}(0)} d^{2} x M_{\mu}(x) f_{\mu}(x)\right|=\frac{2 \pi N^{-1+2 \mu}}{r}\left|\int_{r_{0}}^{r} d r^{\prime} r^{\prime} f_{\mu}\left(r^{\prime}\right)\right| \\
& \leq \frac{2 \pi N^{-1+2 \mu}}{r}\left|\int_{r_{0}}^{r} d r^{\prime} r^{\prime}\left(r^{\prime}-r_{0}\right) \sup _{r_{0} \leq s \leq r}\right| f_{\mu}^{\prime}(s)|| .
\end{aligned}
$$

Taking the supreme over the interval $\left[r_{0}, r\right]$, the inequality above then implies that there exists a constant $C\left(r, r_{0}\right) \neq 0, \lim _{r \rightarrow r_{0}} C\left(r, r_{0}\right)=0$ such that

$$
\sup _{r_{0} \leq s \leq r}\left|f_{\mu}^{\prime}(s)\right| \leq C\left(r, r_{0}\right) N^{-1+2 \mu} \sup _{r_{0} \leq s \leq r}\left|f_{\mu}^{\prime}(s)\right| .
$$


Thus, for $r$ close enough to $r_{0}$, the inequality above can only hold if $f_{\mu}^{\prime}(s)=0$ for $s \in\left[r_{0}, r\right]$, yielding a contradiction to the choice of $r_{0}$.

Consequently, there exists a $x_{0} \in \mathbb{R}^{2},\left|x_{0}\right| \leq N^{-\mu}$, such that $f_{\mu}\left(x_{0}\right) \neq 0$. We can thus define

$$
h(x)=f_{\mu}(x) \frac{j_{N, R}\left(x_{0}\right)}{f_{\mu}\left(x_{0}\right)}
$$

on the compact set $\overline{B_{x_{0}}(0)}$. One easily sees that $h(x)=j_{N, R}(x)$ on $\partial \overline{B_{x_{0}}(0)}$ and satisfies the zero energy scattering equation (26) for $x \in \overline{B_{N^{-\mu}}(0)}$. Note that the scattering equations (26) and (30) have a unique solution on any compact set. It then follows that $h(x)=j_{N, R}(x) \forall x \in \overline{B_{N^{-\mu}}(0)}$. Since $j_{N, R}\left(N^{-\mu}\right) \neq 0$, we then obtain $f_{\mu}\left(N^{-\mu}\right) \neq 0$. Applying Theorem C.1 in [38] once more, it then follows that either $f_{\mu}$ or $-f_{\mu}$ is a nonnegative, monotone nondecrasing function in $|x|$ for all $|x| \leq N^{-\mu}$.

Recall that $M_{\mu}$ and hence $f_{\mu}(x)$ depend on $R_{\mu} \in\left[N^{-\mu}, \infty\right.$ [. For conceptual clarity, we denote $M_{\mu}^{\left(R_{\mu}\right)}(x)=M_{\mu}(x)$ and $f_{\mu}^{\left(R_{\mu}\right)}(x)=f_{\mu}(x)$ for the rest of the proof of part (a). For $\mu$ fixed, consider the function

$$
\begin{aligned}
& s:\left[N^{-\mu}, \infty[\rightarrow \mathbb{R}\right. \\
& R_{\mu} \mapsto \int_{B_{R_{\mu}}(0)} d^{2} x\left(V_{N}(x)-M_{\mu}^{\left(R_{\mu}\right)}(x)\right) f_{\mu}^{\left(R_{\mu}\right)}(x) .
\end{aligned}
$$

We show by contradiction that the function $s$ has at least one zero. Assume $s \neq 0$ were to hold. We can assume w.l.o.g. $s>0$. It then follows from Gauss'-theorem that $f_{\mu}^{\prime\left(R_{\mu}\right)}\left(R_{\mu}\right)>0$ for all $R_{\mu} \geq N^{-\mu}$. By uniqueness of the solution of the scattering equation (30), for $\tilde{R}_{\mu}<R_{\mu}$ there exists a constant $K_{\tilde{R}_{\mu}, R_{\mu}} \neq 0$, such that for all $|x| \leq \tilde{R}_{\mu}$ we have $f_{\mu}^{\left(\tilde{R}_{\mu}\right)}(x)=K_{\tilde{R}_{\mu}, R_{\mu}} f_{\mu}^{\left(R_{\mu}\right)}(x)$. Since $f_{\mu}^{\left(R_{\mu}\right)}$ and $s$ are continuous, we can further conclude $K_{\tilde{R}_{\mu}, R_{\mu}}>0$. From $s \neq 0$, it then follows that, for all $r \in\left[N^{-\mu}, \infty\left[\right.\right.$ and for all $R_{\mu} \in\left[N^{-\mu}, \infty\left[, f_{\mu}^{\prime\left(R_{\mu}\right)}(r) \neq 0\right.\right.$. Thus, for all $r \in\left[N^{-\mu}, \infty\left[\right.\right.$ and for all $R_{\mu} \in\left[N^{-\mu_{1}}, \infty\left[\right.\right.$, the function $f_{\mu}^{\left(R_{\mu}\right)}(r)$ doesn't change sign. From Lemma 5.2, the assumption $s\left(N^{-\mu}\right)>0$ and $K_{\tilde{R}_{\mu}, R_{\mu}}>0$, we obtain, for all $r \in\left[0, N^{-\mu}\right]$ and for all $R_{\mu} \in\left[N^{-\mu}, \infty\left[\right.\right.$, that $f_{\mu}^{\left(R_{\mu}\right)}(r) \geq 0$ holds. This, however, implies $\lim _{R_{\mu} \rightarrow \infty} s\left(R_{\mu}\right)=-\infty$ yielding to a contradiction. By continuity of $s$, there exists thus a minimal value $R_{\mu} \geq N^{-\mu}$ such that $s\left(R_{\mu}\right)=0$.

Remark 5.6. As mentioned, we will from now on fix $R_{\mu} \in\left[N^{-\mu}, \infty[\right.$ as the minimal value such that $s\left(R_{\mu}\right)=0$. Furthermore, we may assume $a>0$ and $R_{\mu}>N^{-\mu}$ in the following. For $a=0$, we can choose $R_{\mu}=N^{-\mu}$, such that $f_{\mu}(x)=j_{N, R}(x)$. It is then easy to verify that the Lemma stated is valid.

(b) From (a), we can conclude that

$$
K_{\mu}=\frac{j_{N, R_{\mu}}\left(N^{-\mu}\right)}{f_{\mu}\left(N^{-\mu}\right)} .
$$

Next, we show that the constant $K_{\mu}$ is positive. Since $j_{N, R_{\mu}}\left(N^{-\mu}\right)$ is positive, it follows from Eq. (35) that $K_{\mu}$ and $f_{\mu}\left(N^{-\mu}\right)$ have equal sign. By (a), the sign of $f_{\mu}$ 
is constant for $|x| \leq R_{\mu}$. Since $j_{N, R_{\mu}}$ and $V_{N}$ are nonnegative functions, we obtain by Gauss-theorem and the scattering equation (30)

$$
\operatorname{sgn}\left(\left.\frac{\partial f_{\mu}}{\partial r}\right|_{r=N^{-\mu}}\right)=\operatorname{sgn}\left(K_{\mu}\right)
$$

Recall that $R_{\mu}$ is the smallest value such that $\left.\frac{\partial f_{\mu}}{\partial r}\right|_{r=R_{\mu}}=0$. If it were now that $K_{\mu}$ is negative, we could conclude from (35) and (36) that $\left.\frac{\partial f_{\mu}}{\partial r}\right|_{r=N^{-\mu}}<0$ and $f_{\mu}\left(N^{-\mu}\right)<0$. Since $R_{\mu}$ is by definition the smallest value where $\frac{\partial f_{\mu}}{\partial r}=0$, we were able to conclude from the continuity of the derivative that $\frac{\partial f_{\mu}}{\partial r}<0$ for all $r<R_{\mu}$ and hence $f\left(R_{\mu}\right)<0$. However, this were in contradiction to the boundary condition of the zero energy scattering state [see (30)] and thus $K_{\mu}>0$ follows.

(c) This directly follows from $e^{-N}<C N^{-\mu}$ for $N$ sufficiently large.

(d) From the proof of property (b), we see that $f_{\mu}$ and its derivative is positive at $N^{-\mu}$. From (34), we obtain $f_{\mu}^{\prime}(r)=0$ for all $r>R_{\mu}$. Further (34) gives that $R_{\mu}$ is the smallest value such that $f_{\mu}^{\prime}\left(R_{\mu}\right)=0$. This and continuity imply that $f_{\mu}^{\prime}(r)>0$ for all $r<R_{\mu}$. Since $f_{\mu}$ is continuous, positive at $N^{-\mu}$, and its derivative is a nonnegative function, it follows that $f_{\mu}$ is a positive, monotone nondecreasing function in $|x|$.

(e) By definition of $R_{\mu}$, it follows that $\tilde{I}=\int_{\mathbb{R}^{2}} d^{2} x\left(V_{N}(x)-M_{\mu}(x)\right) f_{\mu}(x)=0$. Therefore, for all $|x| \geq R_{\mu}, f_{\mu}$ solves $-\Delta f_{\mu}(x)=0$, which has the solution

$$
f_{\mu}(x)=1+\frac{\tilde{I}}{4 \pi} \ln \left(\frac{|x|}{R_{\mu}}\right)=1 .
$$

(f) Since $f_{\mu}$ is a positive monotone nondecreasing function in $|x|$, we obtain

$$
1 \geq f_{\mu}\left(N^{-\mu}\right)=j_{N, R_{\mu}}\left(N^{-\mu}\right) / K_{\mu}=\left(1+\frac{1}{N+\ln \left(\frac{R_{\mu}}{a}\right)} \ln \left(\frac{N^{-\mu}}{R_{\mu}}\right)\right) / K_{\mu} .
$$

We obtain the lower bound

$$
K_{\mu} \geq 1+\frac{1}{N+\ln \left(\frac{R_{\mu}}{a}\right)} \ln \left(\frac{N^{-\mu}}{R_{\mu}}\right) .
$$

For the upper bound we first prove that $f_{\mu}(x) \geq j_{N, R_{\mu}}(x)$ holds for all $|x| \leq R_{\mu}$. Using the scatting equations (26) and (30) we obtain

$$
\Delta_{x}\left(f_{\mu}(x)-j_{N, R_{\mu}}(x)\right)=\frac{1}{2} V_{N}(x)\left(f_{\mu}(x)-j_{N, R_{\mu}}(x)\right)-\frac{1}{2} M_{\mu}(x) f_{\mu}(x)
$$

as well as $f_{\mu}\left(R_{\mu}\right)-j_{N, R_{\mu}}\left(R_{\mu}\right)=0$. Since $M_{\mu}(x) f_{\mu}(x) \geq 0$, we obtain that $\Delta_{x}\left(f_{\mu}(x)-j_{N, R_{\mu}}(x)\right) \leq 0$ for $N^{-\mu} \leq|x| \leq R_{\mu}$. That is, $f_{\mu}(x)-j_{N, R_{\mu}}(x)$ is superharmonic for $N^{-\mu}<|x|<R_{\mu}$. Using the minimum principle, we obtain, using that $f_{\mu}-j_{N, R_{\mu}}$ is spherically symmetric

$$
\min _{N^{-\mu} \leq|x| \leq R_{\mu}}\left(f_{\mu}-j_{N, R_{\mu}}\right)=\min _{|x| \in\left\{N^{-\mu}, R_{\mu}\right\}}\left(f_{\mu}-j_{N, R_{\mu}}\right) .
$$


If it were now that $\min _{|x| \in\left\{N^{-\mu}, R_{\mu}\right\}}\left(f_{\mu}-j_{N, R_{\mu}}\right)=f_{\mu}\left(N^{-\mu}\right)-j_{N, R_{\mu}}\left(N^{-\mu}\right) \leq$ $f_{\mu}\left(R_{\mu}\right)-j_{N, R_{\mu}}\left(R_{\mu}\right)=0$, we could conclude that $f_{\mu}(x)-j_{N, R_{\mu}}(x) \leq 0$ for all $N^{-\mu} \leq|x| \leq R_{\mu}$. Since $f_{\mu}(x)-j_{N, R_{\mu}}(x)$ then obeys

$$
\begin{cases}-\Delta\left(f_{\mu}(x)-j_{N, R_{\mu}}(x)\right)+\frac{1}{2} V_{N}(x)\left(f_{\mu}(x)-j_{N, R_{\mu}}(x)\right)=0 & \text { for }|x| \leq N^{-\mu} \\ f_{\mu}(x)-j_{N, R_{\mu}}(x) \leq 0 & \text { for }|x|=N^{-\mu}\end{cases}
$$

we could then conclude that $f_{\mu}(x)-j_{N, R_{\mu}}(x) \leq 0$ for all $|x| \leq R_{\mu}$. From this, we obtain that $\Delta\left(f_{\mu}(x)-j_{N, R_{\mu}}(x)\right) \leq 0$ for $|x| \leq R_{\mu}$. That is, $f_{\mu}(x)-j_{N, R_{\mu}}(x)$ is superharmonic for all $|x| \leq R_{\mu}$. Using the minimum principle once again, we then obtain

$$
\frac{\min }{B_{R_{\mu}}(0)}\left(f_{\mu}-j_{N, R_{\mu}}\right)=f_{\mu}\left(R_{\mu}\right)-j_{N, R_{\mu}}\left(R_{\mu}\right)=0
$$

which contradicts $f_{\mu}(x)-j_{N, R_{\mu}}(x) \leq 0$ for $|x| \leq R_{\mu}$. Therefore, we can conclude in (37) that $\min _{N^{-\mu} \leq|x| \leq R_{\mu}}\left(f_{\mu}-j_{N, R_{\mu}}\right)=f_{\mu}\left(R_{\mu}\right)-j_{N, R_{\mu}}\left(R_{\mu}\right)=0$ holds. Then, it follows that $f_{\mu}(x)-j_{N, R_{\mu}}(x) \geq 0$ for all $N^{-\mu} \leq|x| \leq R_{\mu}$. Using the zero energy scattering equation $-\Delta\left(f_{\mu}(x)-j_{N, R_{\mu}}(x)\right)+\frac{1}{2} V_{N}(x)\left(f_{\mu}(x)-j_{N, R_{\mu}}(x)\right)=0$ for $|x| \leq N^{-\mu}$, we can, together with $f_{\mu}\left(N^{-\mu}\right)-j_{N, R_{\mu}}\left(N^{-\mu}\right) \geq 0$, conclude that $f_{\mu}(x)-j_{N, R_{\mu}}(x) \geq 0$ for all $|x| \leq R_{\mu}$.

As a consequence, we obtain the desired bound $K_{\mu}=\frac{j_{N, R_{\mu}}\left(N^{-\mu}\right)}{f_{\mu}\left(N^{-\mu}\right)} \leq 1$.

(g) Since $f_{\mu}$ is a nonnegative, monotone nondecreasing function in $|x|$ with $f_{\mu}(x)=1$ $\forall|x| \geq R_{\mu}$, it follows that

$$
\begin{aligned}
C f_{\mu}\left(N^{-\mu}\right) & =f_{\mu}\left(N^{-\mu}\right) \int_{\mathbb{R}^{2}} d^{2} x V_{N}(x) \geq \int_{\mathbb{R}^{2}} d^{2} x V_{N}(x) f_{\mu}(x) \\
& =\int_{\mathbb{R}^{2}} d^{2} x M_{\mu}(x) f_{\mu}(x) \geq f_{\mu}\left(N^{-\mu}\right) \int_{\mathbb{R}^{2}} d^{2} x M_{\mu}(x) .
\end{aligned}
$$

Therefore, $\int_{\mathbb{R}^{2}} d^{2} x M_{\mu}(x) \leq C$ holds, which implies that $R_{\mu} \leq C N^{1 / 2-\mu}$. From

$$
\begin{aligned}
\frac{1}{K_{\mu}} \frac{4 \pi}{N+\ln \left(\frac{R_{\mu}}{a}\right)} & =\frac{1}{K_{\mu}} \int_{\mathbb{R}^{2}} d^{2} x V_{N}(x) j_{N, R_{\mu}}(x)=\int_{\mathbb{R}^{2}} d^{2} x V_{N}(x) f_{\mu}(x) \\
& =\int_{\mathbb{R}^{2}} d^{2} x M_{\mu}(x) f_{\mu}(x)=8 \pi^{2} N^{-1+2 \mu} \int_{N^{-\mu}}^{R_{\mu}} d r r f_{\mu}(r)
\end{aligned}
$$

we conclude that

$$
\int_{N^{-\mu}}^{R_{\mu}} \operatorname{drrf} f_{\mu}(r)=\frac{N^{1-2 \mu}}{2 \pi K_{\mu}\left(N+\ln \left(\frac{R_{\mu}}{a}\right)\right)}
$$

Since $f_{\mu}$ is a nonegative, monotone nondecreasing function in $|x|$,

$$
\frac{1}{2}\left(R_{\mu}^{2}-N^{-2 \mu}\right) \frac{j_{N, R_{\mu}}\left(N^{-\mu}\right)}{K_{\mu}}=\frac{1}{2}\left(R_{\mu}^{2}-N^{-2 \mu}\right) f_{\mu}\left(N^{-\mu}\right) \leq \int_{N^{-\mu}}^{R_{\mu}} d r r f_{\mu}(r)
$$


which implies

$$
R_{\mu}^{2} N^{2 \mu} \leq \frac{N}{\pi\left(N+\ln \left(\frac{R_{\mu}}{a}\right)\right) j_{N, R_{\mu}}\left(N^{-\mu}\right)}+1
$$

Using $R_{\mu} \leq C N^{1 / 2-\mu}$, it then follows

$$
j_{N, R_{\mu}}\left(N^{-\mu}\right)=1+\frac{1}{N+\ln \left(\frac{R_{\mu}}{a}\right)} \ln \left(\frac{N^{-\mu}}{R_{\mu}}\right) \geq 1-\frac{C}{N},
$$

which implies $R_{\mu} \leq C N^{-\mu}$.

(h) Using

$$
\left\|M_{\mu} f_{\mu}\right\|_{1}=\left\|V_{N} f_{\mu}\right\|_{1}=K_{\mu}^{-1}\left\|V_{N} j_{N, R_{\mu}}\right\|_{1}=K_{\mu}^{-1} \frac{4 \pi}{N+\ln \left(\frac{R_{\mu}}{a}\right)},
$$

we obtain

$$
\begin{aligned}
|N|\left\|V_{N} f_{\mu}\right\|_{1}-4 \pi \mid & =\left|N\left\|M_{\mu} f_{\mu}\right\|_{1}-4 \pi\right|=4 \pi\left|K_{\mu}^{-1} \frac{N}{N+\ln \left(\frac{R_{\mu}}{a}\right)}-1\right| \\
& =\frac{4 \pi}{K_{\mu}}\left|\frac{N-N K_{\mu}+K_{\mu} \ln \left(\frac{R_{\mu}}{a}\right)}{N+\ln \left(\frac{R_{\mu}}{a}\right)}\right| \leq C \frac{\ln (N)}{N} .
\end{aligned}
$$

(i) Using for $|x| \leq R_{\mu}$ the inequalities $j_{N, R_{\mu}}(x) \geq 1+\frac{1}{N+\ln \left(\frac{R_{\mu}}{a}\right)} \ln \left(\frac{|x|}{R_{\mu}}\right)$ as well as $1 \geq f_{\mu}(x) \geq j_{N, R_{\mu}}(x)$, it follows for $|x| \leq R_{\mu}$

$$
\begin{aligned}
0 & \leq g_{\mu}(x)=1-f_{\mu}(x) \leq 1-j_{N, R_{\mu}}(x) \leq-\frac{1}{N+\ln \left(\frac{R_{\mu}}{a}\right)} \ln \left(\frac{|x|}{R_{\mu}}\right) \\
& \leq C N^{-1}|\ln (N|x|)| .
\end{aligned}
$$

Since $g_{\mu}(x)=0$ for $|x|>R_{\mu}$, we conclude with $R_{\mu} \leq C N^{-\mu}$ that

$$
\left\|g_{\mu}\right\|_{1} \leq \frac{C}{N} \int_{0}^{R_{\mu}} d r r|\ln (N r)| \leq C N^{-1-2 \mu} \ln N,
$$

as well as

$$
\begin{aligned}
\left\|g_{\mu}\right\|^{2} & \leq \frac{C}{N^{2}} \int_{0}^{R_{\mu}} d r r(\ln (N r))^{2} \\
& =C N^{-4}\left[r^{2}\left(2(\ln (r))^{2}-2 \ln (r)+1\right)\right]_{0}^{N R_{\mu}} \\
& \leq C N^{-2-2 \mu}(\ln (N))^{2} .
\end{aligned}
$$

$\left\|g_{\mu}\right\|_{\infty}=\left\|1-f_{\mu}\right\|_{\infty} \leq 1$, since $f_{\mu}$ is a nonnegative, monotone nondecreasing function with $f_{\mu}(x) \leq 1$. 
(j) Using (h) and (i), we obtain with $\left\|M_{\mu}\right\|_{1} \leq C N^{-1}$

$$
\begin{aligned}
\left|N\left\|M_{\mu}\right\|_{1}-4 \pi\right| & \leq\left|N\left\|M_{\mu} f_{\mu}\right\|_{1}-4 \pi\right|+N\left\|M_{\mu} g_{\mu}\right\|_{1} \\
& \leq C\left(\frac{\ln (N)}{N}+\left\|\mathbb{1}_{|\cdot| \geq N^{-\mu}} g_{\mu}\right\|_{\infty}\right) .
\end{aligned}
$$

Since $g_{\mu}(x)$ is a nonnegative, monotone nonincreasing function, it follows with $K_{\mu} \leq 1$

$$
\begin{aligned}
\left\|\mathbb{1}_{|\cdot| \geq N^{-\mu}} g_{\mu}\right\|_{\infty} & =g_{\mu}\left(N^{-\mu}\right)=1-f_{\mu}\left(N^{-\mu}\right)=1-\frac{j_{N, R_{\mu}}\left(N^{-\mu}\right)}{K_{\mu}} \\
& \leq 1-\left(1+\frac{1}{N+\ln \left(\frac{R_{\mu}}{a}\right)} \ln \left(\frac{N^{-\mu}}{R_{\mu}}\right)\right) .
\end{aligned}
$$

and (j) follows.

(k) $M_{\mu} \in \widetilde{\mathcal{W}}_{\mu}$ follows directly from $R_{\mu} \leq C N^{-\mu}$. From part (j) we then get $b_{M_{\mu}}=4 \pi$ and $M_{\mu} \in \mathcal{W}_{\mu}$. By means of part (d) we conclude $0 \leq M_{\mu}(x) f_{\mu}(x) \leq M_{\mu}(x)$ which together with part (h) implies $M_{\mu} f_{\mu} \in \widetilde{\mathcal{W}}_{\mu}, b_{M_{\mu} f_{\mu}}=4 \pi$ and $M_{\mu} f_{\mu} \in \mathcal{W}_{\mu}$.

\section{Proof of the Theorem}

In this section, we present the proof of Theorem 2.4. We start with the NLS regime and then pursue with the exponential scaling. In both cases we follow the same strategy: After giving the precise definition of the functional we explain its connection to the notion of Bose-Einstein condensation in terms of reduced density matrices. Thereupon, we differentiate the functional with respect to its time variable, perform a Grönwall estimate and finally prove the respective part of the theorem.

\subsection{Prooffor the NLS scaling $W_{\beta}$ with $\beta>0$.}

6.1.1. Definition of the functional The goal of this section is to define a functional $\alpha: L^{2}\left(\mathbb{R}^{2 N}, \mathbb{C}\right) \times L^{2}\left(\mathbb{R}^{2}, \mathbb{C}\right) \rightarrow \mathbb{R}_{0}^{+}$which is adapted to potentials with NLS scaling and which meets all the requirements stated in Sect. 3. In short, we demand the functional to converge to zero for properly chosen initial states and its time derivative to be controllable by means of a Grönwall estimate. Additionally, the functional should allow to prove both Bose-Einstein condensation and the convergence of the energy per particle of the manybody system to the effective energy functional.

While interactions in the mean-field scaling ( $W_{\beta}$ with $\beta=0$ ) become weak for large particle numbers, potentials $W_{\beta}$ with $\beta>1 / 2$ are getting peaked as $N \rightarrow \infty$. This fact needs to be taken into account when defining a suitable counting functional. For small $\beta$ and a large class of different choices of the weight $\widehat{m}^{\varphi_{t}}$ with $\varphi_{t}$ being a solution of (3), it is possible to show that

$$
\begin{aligned}
& \left\langle\left\langle\Psi_{t}, \widehat{m}^{\varphi_{t}} \Psi_{t}\right\rangle\right\rangle \leq\left\langle\left\langle\Psi_{0}, \widehat{m}^{\varphi_{0}} \Psi_{0}\right\rangle+\int_{0}^{t} d s\right. \\
& \left(\mathcal { K } ( \varphi _ { s } , A _ { s } ) \left(\left\langle\left\langle\Psi_{s}, \widehat{m}^{\varphi_{s}} \Psi_{s}\right\rangle+\mathcal{O}(1)+\left\langle\left\langle\Psi_{s}, \widehat{n}^{\varphi_{s}} \Psi_{s}\right\rangle+\left|\mathcal{E}_{W_{\beta}}\left(\Psi_{s}\right)-\mathcal{E}_{b_{W_{\beta}}}^{G P}\left(\varphi_{s}\right)\right|\right)\right) .\right.\right.
\end{aligned}
$$


This enables us to perform an integral type Grönwall estimate if we choose

$$
\alpha\left(\Psi_{t}, \varphi_{t}\right)=\left\langle\Psi_{t}, \widehat{n}^{\varphi_{t}} \Psi_{t}\right\rangle+\left|\mathcal{E}_{W_{\beta}}\left(\Psi_{t}\right)-\mathcal{E}_{b_{W_{\beta}}}^{G P}\left(\varphi_{t}\right)\right| .
$$

Here, the smallness of the distance between the energies is used to control the kinetic energy per particle of the many-body system (Lemma 7.6). This prevents the wave function from being strongly localized in the support of the potential and in this way softens the effect of the interaction. Moreover, it allows us to bound the kinetic energy of the particles which are not in the condensate state $\varphi_{t}$ by $\alpha\left(\Psi_{t}, \varphi_{t}\right)$, see Lemma 7.9.

For large $\beta$, the interaction is harder to control and several estimates break down, if one defines $\alpha$ as above. It is therefore necessary to redefine the functional $\alpha\left(\Psi_{t}, \varphi_{t}\right)$ and to carefully choose a new weight function $m$. Let us explain why this is necessary. To obtain an integral type Grönwall estimate, we will calculate the time derivative of $\left\langle\left\langle\Psi_{t}, \widehat{m}^{\varphi_{t}} \Psi_{t}\right\rangle\right\rangle$. This time derivative will contain contributions of the form $\widehat{m}-\widehat{m}_{1}$ and $\widehat{m}-\widehat{m}_{2}$. To obtain sufficient error estimates for large $\beta$, it is necessary to choose a weight function $m$ such that $\left\|\widehat{m}-\widehat{m}_{i}\right\|_{\text {op }}$ with $i=1,2$ can be controlled sufficiently well (one can infer from the proof below that $\left\|\widehat{n}-\widehat{n}_{i}\right\|_{\text {op }}=\mathcal{O}\left(N^{-1 / 2}\right.$ ) with $i=1,2$ is not decaying sufficiently in $N$, see part (b) of Lemma 7.7). For the Grönwall estimate, we require in addition $\|\widehat{m}-\widehat{n}\|_{\text {op }} \rightarrow 0$, as $N \rightarrow \infty$.

In total, this suggests the following form of the functional

Definition 6.1. For $0<\xi<\frac{1}{3}$ define

$$
m(k)= \begin{cases}\sqrt{k / N}, & \text { for } k \geq N^{1-2 \xi} \\ 1 / 2\left(N^{-1+\xi} k+N^{-\xi}\right), & \text { else. }\end{cases}
$$

and

$$
\alpha^{<}(\Psi, \varphi)=\left\langle\left\langle\Psi, \widehat{m}^{\varphi} \Psi\right\rangle+\left|\mathcal{E}_{W_{\beta}}(\Psi)-\mathcal{E}_{b_{W_{\beta}}}^{G P}(\varphi)\right| .\right.
$$

Remark 6.2. It should be noted, that $\alpha^{<}$depends on the parameter $\xi$ which will be chosen later. For better readability, we disregard the $\xi$ dependence in the notation.

The counting measure can be related to the trace norm distance of the one-particle reduced density matrix.

Lemma 6.3. Let $0<\xi<1 / 3, \Psi \in L_{s}^{2}\left(\mathbb{R}^{2 N}, \mathbb{C}\right), \varphi \in L^{2}\left(\mathbb{R}^{2}, \mathbb{C}\right)$ and $\alpha^{<}(\Psi, \varphi)$ be defined as in Definition 6.1. Then,

$$
\begin{aligned}
\left.\operatorname{Tr}\left|\gamma_{\Psi}^{(1)}-\right| \varphi\right\rangle\langle\varphi|| & \leq \sqrt{8 \alpha^{<}(\Psi, \varphi)}, \\
\alpha^{<}(\Psi, \varphi) & \leq \sqrt{\left.\operatorname{Tr}\left|\gamma_{\Psi}^{(1)}-\right| \varphi\right\rangle\langle\varphi||}+\left|\mathcal{E}_{W_{\beta}}(\Psi)-\mathcal{E}_{b_{W_{\beta}}}^{G P}(\varphi)\right|+\frac{1}{2} N^{-\xi} .
\end{aligned}
$$

Proof. We would like to mention, that this Lemma has been proven in [6, Lemma 3.3]. For sake of completeness, we briefly recall the argument. From [30, Lemma2.3] and $[50$, eq. (6)] one concludes

$$
\left.\left.\| \Psi,\left(\widehat{n}^{\varphi}\right)^{2} \Psi\right\rangle \leq \operatorname{Tr}\left|\gamma_{\Psi}^{(1)}-\right| \varphi\right\rangle\left\langle\varphi|| \leq \sqrt{8\left\langle\Psi,\left(\widehat{n}^{\varphi}\right)^{2} \Psi\right\rangle} .\right.
$$

If one then uses that $n(k)^{2} \leq n(k) \leq m(k)$ and $m(k) \leq n(k)+\frac{1}{2} N^{-\xi}$ imply the relations

$$
\left.\| \Psi,\left(\widehat{n}^{\varphi}\right)^{2} \Psi\right\rangle \leq\left\langle\langle \Psi , \widehat { m } ^ { \varphi } \Psi \rangle \text { and } \left\langle\left\langle\Psi, \widehat{m}^{\varphi} \Psi\right\rangle \leq \sqrt{\left\langle\Psi,\left(\widehat{n}^{\varphi}\right)^{2} \Psi\right\rangle}+\frac{1}{2} N^{-\xi},\right.\right.
$$

the Lemma follows. 
6.1.2. Preliminaries for the Grönwall estimate Subsequently, we will perform a Grönwall estimate for $\alpha^{<}$and prove part (a) of Theorem 2.4. For this, we define

Definition 6.4. Let $0<\xi<1 / 3$ and $W_{\beta} \in \mathcal{W}_{\beta}$. Define

$$
Z_{\beta}^{\varphi}\left(x_{j}, x_{k}\right)=W_{\beta}\left(x_{j}-x_{k}\right)-\frac{N\left\|W_{\beta}\right\|_{1}}{N-1}|\varphi|^{2}\left(x_{j}\right)-\frac{N\left\|W_{\beta}\right\|_{1}}{N-1}|\varphi|^{2}\left(x_{k}\right)
$$

Note, for $W_{\beta}(x)=N^{-1+2 \beta} W\left(N^{\beta} x\right)$, we have $N\left\|W_{\beta}\right\|_{1}=\|W\|_{1}$. With

$$
m^{a}(k)=m(k)-m(k+1), \quad m^{b}(k)=m(k)-m(k+2)
$$

and

$$
\widehat{r}=\widehat{m}^{b} p_{1} p_{2}+\widehat{m}^{a}\left(p_{1} q_{2}+q_{1} p_{2}\right),
$$

we define for $l \in\{a, b, c\}$ the functionals $\gamma_{l}^{<}: L^{2}\left(\mathbb{R}^{2 N}, \mathbb{C}\right) \times L^{2}\left(\mathbb{R}^{2}, \mathbb{C}\right) \rightarrow \mathbb{R}_{0}^{+}$by

$$
\begin{aligned}
\gamma_{a}^{<}(\Psi, \varphi)= & \left\langle\Psi \Psi, \dot{A}_{t}\left(x_{1}\right) \Psi\right\rangle-\left\langle\varphi, \dot{A}_{t} \varphi\right\rangle \\
\gamma_{b}^{<}(\Psi, \varphi)= & N(N-1) \mathfrak{s}\left(\left\langle\Psi, Z_{\beta}^{\varphi}\left(x_{1}, x_{2}\right) \widehat{r} \Psi\right\rangle\right) \\
= & -2 N(N-1) \Im\left(\left\langle\Psi, p_{1} q_{2} \widehat{m}_{-1}^{a} Z_{\beta}^{\varphi}\left(x_{1}, x_{2}\right) p_{1} p_{2} \Psi\right\rangle\right) \\
& -N(N-1) \Im\left(\left\langle\Psi, q_{1} q_{2} \widehat{m}_{-2}^{b} W_{\beta}\left(x_{1}-x_{2}\right) p_{1} p_{2} \Psi\right\rangle\right) \\
& -2 N(N-1) \mathfrak{s}\left(\left\langle\Psi, q_{1} q_{2} \widehat{m}_{-1}^{a} Z_{\beta}^{\varphi}\left(x_{1}, x_{2}\right) p_{1} q_{2} \Psi\right\rangle\right) \\
\gamma_{c}^{<}(\Psi, \varphi)= & i N\left(N\left\|W_{\beta}\right\|_{1}-b_{W_{\beta}}\right)\left\langle\Psi \Psi,\left(q_{1}\left|\varphi\left(x_{1}\right)\right|^{2} \widehat{m}^{a} p_{1}-p_{1} \widehat{m}^{a}\left|\varphi\left(x_{1}\right)\right|^{2} q_{1}\right) \Psi\right\rangle .
\end{aligned}
$$

The value of the functional $\alpha^{<}\left(\Psi_{t}, \varphi_{t}\right)$ at time $t$ is then bounded by

Lemma 6.5. Let $W_{\beta} \in \mathcal{W}_{\beta}$. Let $\Psi_{t}$ the unique solution to $i \partial_{t} \Psi_{t}=H_{W_{\beta}} \Psi_{t}$ with initial datum $\Psi_{0} \in L_{s}^{2}\left(\mathbb{R}^{2 N}, \mathbb{C}\right) \cap H^{2}\left(\mathbb{R}^{2 N}, \mathbb{C}\right),\left\|\Psi_{0}\right\|=1$. Let $\varphi_{t}$ the unique solution to $i \partial_{t} \varphi_{t}=h_{b_{W_{\beta}}}^{G P} \varphi_{t}$ with $\varphi_{t} \in H^{3}\left(\mathbb{R}^{2}, \mathbb{C}\right),\left\|\varphi_{0}\right\|=1$. Let $\alpha<\left(\Psi_{t}, \varphi_{t}\right)$ be defined as in Definition 6.1. Then

$$
\alpha^{<}\left(\Psi_{t}, \varphi_{t}\right) \leq \alpha^{<}\left(\Psi_{0}, \varphi_{0}\right)+\int_{0}^{t} d s\left(\left|\gamma_{a}^{<}\left(\Psi_{s}, \varphi_{s}\right)\right|+\left|\gamma_{b}^{<}\left(\Psi_{s}, \varphi_{s}\right)\right|+\left|\gamma_{c}^{<}\left(\Psi_{s}, \varphi_{s}\right)\right|\right) \text {. }
$$

Proof. For the proof of the Lemma we restore the upper index $\varphi_{t}$ in order to pay respect to the time dependence of $\widehat{m}^{\varphi_{t}}$. The time derivative of $\varphi_{t}$ is given by (3), i.e. $i \partial_{t} \varphi_{t}\left(x_{j}\right)=$ $h_{b_{W_{\beta}}, j}^{G P} \varphi_{t}\left(x_{j}\right)$. Here, $h_{b_{W_{\beta}}, j}^{G P}$ denotes the operator $h_{b_{W_{\beta}}}^{G P}$ acting on the $j^{\text {th }}$ coordinate $x_{j}$. 
We then obtain

$$
\begin{aligned}
\frac{d}{d t}\left\langle\left\langle\Psi_{t}, \widehat{m}^{\varphi_{t}} \Psi_{t}\right\rangle=\right. & \left.i\left\langle H_{W_{\beta}} \Psi_{t}, \widehat{m}^{\varphi_{t}} \Psi_{t}\right\rangle-i\left\langle\Psi_{t}, \widehat{m}^{\varphi_{t}} H_{W_{\beta}} \Psi_{t}\right\rangle\right\rangle \\
& -i\left\langle\Psi_{t},\left[\sum_{j=1}^{N} h_{b_{W_{\beta}}, j}^{G P}, \widehat{m}^{\varphi_{t}}\right] \Psi_{t}\right\rangle \\
= & i\left\langle\Psi_{t},\left[H_{W_{\beta}}-\sum_{j=1}^{N} h_{b_{W_{\beta}}, j}^{G P}, \widehat{m}^{\varphi_{t}}\right] \Psi_{t}\right\rangle \\
= & \left.i\left\langle\Psi_{t},\left[\left(\frac{1}{2} N(N-1) W_{\beta}\left(x_{1}-x_{2}\right)-N b_{W_{\beta}}\left|\varphi_{t}\left(x_{1}\right)\right|^{2}\right), \widehat{m}^{\varphi_{t}}\right] \Psi_{t}\right\rangle\right\rangle \\
= & i N\left(N\left\|W_{\beta}\right\|_{1}-b_{W_{\beta}}\right)\left\langle\left\langle\Psi_{t},\left[\left|\varphi_{t}\left(x_{1}\right)\right|^{2}, \widehat{m}^{\varphi_{t}}\right] \Psi_{t}\right\rangle\right\rangle \\
& +i \frac{N(N-1)}{2}\left\langle\left\langle\Psi_{t},\left[Z_{\beta}^{\varphi_{t}}\left(x_{1}, x_{2}\right), \widehat{m}^{\varphi_{t}}\right] \Psi_{t}\right\rangle,\right.
\end{aligned}
$$

where we used the symmetry of $\Psi_{t}$. Using Lemma 4.2 (d), it follows that (dropping the explicit dependence on $\varphi_{t}$ from now on)

$$
\begin{aligned}
\frac{d}{d t}\left\langle\left\langle\Psi_{t}, \widehat{m}^{\varphi_{t}} \Psi_{t}\right\rangle=\right. & i N\left(N\left\|W_{\beta}\right\|_{1}-b_{W_{\beta}}\right)\left\langle\left\langle\Psi_{t},\left(q_{1}\left|\varphi_{t}\left(x_{1}\right)\right|^{2} \widehat{m}^{a} p_{1}\right.\right.\right. \\
& \left.\left.-p_{1} \widehat{m}^{a}\left|\varphi_{t}\left(x_{1}\right)\right|^{2} q_{1}\right) \Psi_{t}\right\rangle \\
& +i \frac{N(N-1)}{2}\left\langle\left\langle\Psi_{t},\left[Z_{\beta}^{\varphi_{t}}\left(x_{1}, x_{2}\right), p_{1} p_{2}\left(\widehat{m}-\widehat{m}_{2}\right)\right] \Psi_{t}\right\rangle\right\rangle \\
& +i \frac{N(N-1)}{2}\left\langle\left\langle\Psi_{t},\left[Z_{\beta}^{\varphi_{t}}\left(x_{1}, x_{2}\right),\left(p_{1} q_{2}+q_{1} p_{2}\right)\left(\widehat{m}-\widehat{m}_{1}\right)\right] \Psi_{t}\right\rangle .\right.
\end{aligned}
$$

Since $Z_{\beta}^{\varphi_{t}}$ and $p_{1} p_{2}\left(\widehat{m}-\widehat{m}_{2}\right)$ as well as $p_{1} q_{2}\left(\widehat{m}-\widehat{m}_{1}\right)$ are selfadjoint, we obtain

$$
\begin{aligned}
& \frac{d}{d t}\left\langle\Psi_{t}, \widehat{m}^{\varphi_{t}} \Psi_{t}\right\rangle=\gamma_{c}^{<}\left(\Psi_{t}, \varphi_{t}\right)-N(N-1) \\
& \quad \times \Im\left(\left\langle\Psi_{t},\left(p_{1} p_{2}+p_{1} q_{2}+q_{1} p_{2}+q_{1} q_{2}\right) Z_{\beta}^{\varphi_{t}}\left(x_{1}, x_{2}\right)\left(\widehat{m}^{b} p_{1} p_{2}+\widehat{m}^{a}\left(p_{1} q_{2}+q_{1} p_{2}\right)\right) \Psi_{t}\right\rangle\right) .
\end{aligned}
$$

Note that in view of Lemma 4.2 (c) $\widehat{r} Q_{j} Z_{\beta}^{\varphi_{t}}\left(x_{1}, x_{2}\right) Q_{j}=Q_{j} Z_{\beta}^{\varphi_{t}}\left(x_{1}, x_{2}\right) Q_{j} \widehat{r}$ for any $j \in\{0,1,2\}$ and any weight $r$. Therefore,

$$
\begin{aligned}
\Im\left(\left\langle\Psi_{t}, p_{1} p_{2} Z_{\beta}^{\varphi_{t}}\left(x_{1}, x_{2}\right) \widehat{m}^{b} p_{1} p_{2} \Psi_{t}\right\rangle\right) & =0 \\
\Im\left(\left\langle\Psi_{t},\left(p_{1} q_{2}+q_{1} p_{2}\right) Z_{\beta}^{\varphi_{t}}\left(x_{1}, x_{2}\right) \widehat{m}^{a}\left(p_{1} q_{2}+q_{1} p_{2}\right) \Psi_{t}\right\rangle\right) & =0 .
\end{aligned}
$$

Using Symmetry and Lemma 4.2 (c), we obtain the first line (43). Furthermore,

$$
\begin{aligned}
\frac{d}{d t}\left\langle\left\langle\Psi_{t}, \widehat{m}^{\varphi_{t}} \Psi_{t}\right\rangle=\right. & \gamma_{c}^{<}\left(\Psi_{t}, \varphi_{t}\right)-2 N(N-1) \mathfrak{\Im}\left(\left\langle\Psi_{t}, \widehat{m}_{-1}^{b} p_{1} q_{2} Z_{\beta}^{\varphi_{t}}\left(x_{1}, x_{2}\right) p_{1} p_{2} \Psi_{t}\right\rangle\right) \\
& -N(N-1) \mathfrak{s}\left(\left\langle\Psi_{t}, \widehat{m}_{-2}^{b} q_{1} q_{2} Z_{\beta}^{\varphi_{t}}\left(x_{1}, x_{2}\right) p_{1} p_{2} \Psi_{t}\right\rangle\right) \\
& -2 N(N-1) \mathfrak{\Im}\left(\left\langle\Psi_{t}, p_{1} p_{2} Z_{\beta}^{\varphi_{t}}\left(x_{1}, x_{2}\right) \widehat{m}^{a} p_{1} q_{2} \Psi_{t}\right\rangle\right) \\
& -2 N(N-1) \mathfrak{\Im}\left(\left\langle\Psi_{t}, \widehat{m}_{-1}^{a} q_{1} q_{2} Z_{\beta}^{\varphi_{t}}\left(x_{1}, x_{2}\right) p_{1} q_{2} \Psi_{t}\right\rangle\right) .
\end{aligned}
$$


Since $p_{1} p_{2}\left|\varphi_{t}^{2}\right|\left(x_{1}\right) q_{1} q_{2}=p_{1} p_{2} q_{2}\left|\varphi_{t}^{2}\right|\left(x_{1}\right) q_{1}=0=p_{1} p_{2}\left|\varphi_{t}^{2}\right|\left(x_{2}\right) q_{1} q_{2}$, we can replace $Z_{\beta}^{\varphi_{t}}\left(x_{1}, x_{2}\right)$ in the second line by $W_{\beta}\left(x_{1}-x_{2}\right)$.

The third line equals $2 N(N-1) \Im\left(\left\langle\Psi_{t}, \widehat{m}^{a} p_{1} q_{2} Z_{\beta}^{\varphi_{t}}\left(x_{1}, x_{2}\right) p_{1} p_{2} \Psi_{t}\right\rangle\right)$. Since

$$
m(k-1)-m(k+1)-(m(k)-m(k+1))=m(k-1)-m(k)
$$

it follows that $\widehat{m}_{-1}^{b}-\widehat{m}^{a}=\widehat{m}_{-1}^{a}-(m(0)-m(1)) P_{0}$ and we get

$$
\begin{aligned}
\frac{d}{d t}\left\langle\left\langle\Psi_{t}, \widehat{m}^{\varphi_{t}} \Psi_{t}\right\rangle\right\rangle= & \left.\gamma_{c}^{<}\left(\Psi_{t}, \varphi_{t}\right)-2 N(N-1) \mathfrak{\Im}\left(\left\langle\Psi_{t}, p_{1} q_{2} \widehat{m}_{-1}^{a} Z_{\beta}^{\varphi_{t}}\left(x_{1}, x_{2}\right) p_{1} p_{2} \Psi_{t}\right\rangle\right)\right) \\
& -N(N-1) \mathfrak{\Im}\left(\left\langle\Psi_{t}, q_{1} q_{2} \widehat{m}_{-2}^{b} W_{\beta}\left(x_{1}-x_{2}\right) p_{1} p_{2} \Psi_{t}\right\rangle\right) \\
& \left.-2 N(N-1) \Im\left(\left\langle\Psi_{t}, q_{1} q_{2} \widehat{m}_{-1}^{a} Z_{\beta}^{\varphi_{t}}\left(x_{1}, x_{2}\right) p_{1} q_{2} \Psi_{t}\right\rangle\right)\right) \\
= & \gamma_{b}^{<}\left(\Psi_{t}, \varphi_{t}\right)+\gamma_{c}^{<}\left(\Psi_{t}, \varphi_{t}\right) .
\end{aligned}
$$

For the second summand of $\alpha^{<}\left(\Psi_{t}, \varphi_{t}\right)$ we have

$$
\begin{aligned}
\frac{d}{d t}\left(\mathcal{E}_{W_{\beta}}\left(\Psi_{t}\right)-\mathcal{E}_{b_{W_{\beta}}}^{G P}\left(\varphi_{t}\right)\right)= & \left\langle\Psi_{t}, \dot{A}_{t}\left(x_{1}\right) \Psi_{t}\right\rangle-\left\langle\varphi_{t}, \dot{A}_{t} \varphi_{t}\right\rangle \\
& -i\left\langle\varphi_{t},\left[h_{b_{W_{\beta}}}^{G P},\left(h_{b_{W_{\beta}}}^{G P}-\frac{b_{W_{\beta}}}{2}\left|\varphi_{t}\right|^{2}\right)\right] \varphi_{t}\right\rangle \\
& -\left\langle\varphi_{t}, \frac{b_{W_{\beta}}}{2}\left(\frac{d}{d t}\left|\varphi_{t}\right|^{2}\right) \varphi_{t}\right\rangle \\
= & \left\langle\Psi_{t}, \dot{A}_{t}\left(x_{1}\right) \Psi_{t}\right\rangle-\left\langle\varphi_{t}, \dot{A}_{t} \varphi_{t}\right\rangle \\
& +i\left\langle\varphi_{t},\left[h_{b_{W_{\beta}}}^{G P}, \frac{b_{W_{\beta}}}{2}\left|\varphi_{t}\right|^{2}\right] \varphi_{t}\right\rangle \\
& -i\left\langle\varphi_{t},\left[h_{b_{W_{\beta}}}^{G P}, \frac{b_{W_{\beta}}}{2}\left|\varphi_{t}\right|^{2}\right] \varphi_{t}\right\rangle \\
= & \gamma_{a}^{<}\left(\Psi_{t}, \varphi_{t}\right) .
\end{aligned}
$$

By explicit estimates, one can show that the functions $\gamma_{j}^{<}(\Psi ., \varphi):. \mathbb{R} \rightarrow \mathbb{R}, t \mapsto$ $\gamma_{j}^{<}\left(\Psi_{t}, \varphi_{t}\right)$ with $j \in\{a, b, c\}$ are continuous if $A . \in C^{1}\left(\mathbb{R}, L^{\infty}\left(\mathbb{R}^{2}, \mathbb{R}\right)\right)$. The Lemma then follows using that $|f(x)| \leq|f(0)|+\int_{0}^{x} d y\left|f^{\prime}(y)\right|$ holds for any $f \in C^{1}(\mathbb{R}, \mathbb{R})$.

6.1.3. The Grönwall estimate In order to establish a Grönwall estimate for $\alpha^{<}$, we have to find a suitable bound for the right hand side of (46).

Lemma 6.6. Let $W_{\beta} \in \mathcal{W}_{\beta}$. Let $\Psi_{t}$ the unique solution to $i \partial_{t} \Psi_{t}=H_{W_{\beta}} \Psi_{t}$ with initial datum $\Psi_{0} \in L_{s}^{2}\left(\mathbb{R}^{2 N}, \mathbb{C}\right) \cap H^{2}\left(\mathbb{R}^{2 N}, \mathbb{C}\right),\left\|\Psi_{0}\right\|=1$. Let $\varphi_{t}$ the unique solution to $i \partial_{t} \varphi_{t}=h_{b_{W_{\beta}}}^{G P} \varphi_{t}$ with $\varphi_{t} \in H^{3}\left(\mathbb{R}^{2}, \mathbb{C}\right),\left\|\varphi_{0}\right\|=1$. Let $\mathcal{E}_{W_{\beta}}\left(\Psi_{0}\right) \leq C$.

(a) Let $\beta<1 / 12$. Moreover, let $\alpha^{<}\left(\Psi_{t}, \varphi_{t}\right), \gamma_{a}^{<}\left(\Psi_{t}, \varphi_{t}\right)$ and $\gamma_{b}^{<}\left(\Psi_{t}, \varphi_{t}\right)$ be defined as in Definitions 6.1 and 6.4 with $\xi=1 / 6$. Then

$$
\left|\gamma_{a}^{<}\left(\Psi_{t}, \varphi_{t}\right)+\gamma_{b}^{<}\left(\Psi_{t}, \varphi_{t}\right)+\gamma_{c}^{<}\left(\Psi_{t}, \varphi_{t}\right)\right| \leq \mathcal{K}\left(\varphi_{t}, A_{t}\right)\left(\alpha^{<}\left(\Psi_{t}, \varphi_{t}\right)+N^{-2 \beta} \ln (N)\right) \text {. }
$$


(b) Let $\beta \geq 1 / 12$. Moreover, let $\alpha^{<}\left(\Psi_{t}, \varphi_{t}\right), \gamma_{a}^{<}\left(\Psi_{t}, \varphi_{t}\right)$ and $\gamma_{b}^{<}\left(\Psi_{t}, \varphi_{t}\right)$ be defined as in Definitions 6.1 and 6.4 with $\xi=1 / 10$. Then

$$
\left|\gamma_{a}^{<}\left(\Psi_{t}, \varphi_{t}\right)+\gamma_{b}^{<}\left(\Psi_{t}, \varphi_{t}\right)+\gamma_{c}^{<}\left(\Psi_{t}, \varphi_{t}\right)\right| \leq \mathcal{K}\left(\varphi_{t}, A_{t}\right)\left(\alpha^{<}\left(\Psi_{t}, \varphi_{t}\right)+N^{-1 / 10}\right)
$$

The proof of Lemma 6.6 is given in Sect. 7.3.

At this point, we only consider the most relevant term $\gamma_{b}{ }^{<}\left(\Psi_{t}, \varphi_{t}\right)$ and explain on a heuristic level why it is small. The principle argument follows the ideas and estimates of [49]. The first line in (44) is the most important one. This expression is only small if the correct coupling parameter $b_{W_{\beta}} \approx N\left\|W_{\beta}\right\|_{1}$ is used in the mean-field equation (3). Then,

$$
N p_{1}^{\varphi_{t}} W_{\beta}\left(x_{1}-x_{2}\right) p_{1}^{\varphi_{t}}=N p_{1}^{\varphi_{t}} W_{\beta} *|\varphi|^{2}\left(x_{2}\right) p_{1}^{\varphi_{t}} \rightarrow p_{1}^{\varphi_{t}}|\varphi|^{2}\left(x_{2}\right)\|W\|_{1} p_{1}^{\varphi_{t}}
$$

converges against the mean-field potential, and hence the first expression of (44) is small.

In order to estimate the second and third line of (44), one tries to bound $N^{2}\left\langle\Psi_{t}, q_{1}^{\varphi_{t}}\right.$ $\left.\left.q_{2}^{\varphi_{t}} \widehat{m}_{-2}^{b} W_{\beta}\left(x_{1}-x_{2}\right) p_{1}^{\varphi_{t}} p_{2}^{\varphi_{t}} \Psi_{t}\right\rangle\right\rangle$ and $N^{2}\left\langle\left\langle\Psi_{t}, q_{1}^{\varphi_{t}} q_{2}^{\varphi_{t}} \widehat{m}_{-1}^{a} Z_{\beta}^{\varphi_{1}}\left(x_{1}-x_{2}\right) p_{1}^{\varphi_{t}} q_{2}^{\varphi_{t}} \Psi_{t}\right\rangle\right.$ in terms of $\left\langle\left\langle\Psi_{t}, \widehat{n}^{\varphi_{t}} \Psi_{t}\right\rangle\right\rangle+\mathcal{O}\left(N^{-\eta}\right)$ for some $\eta>0$. By means of

$$
\left|\left\langle\Psi_{t}, \widehat{n}^{\varphi_{t}} \Psi_{t}\right\rangle\right\rangle-\left\langle\left\langle\Psi_{t}, \widehat{m}^{\varphi_{t}} \Psi_{t}\right\rangle\right| \leq\left\|\widehat{n}^{\varphi_{t}}-\widehat{m}^{\varphi_{t}}\right\|_{\text {op }}=N^{-\xi}
$$

this can then be bounded by $\alpha^{<}\left(\Psi_{t}, \varphi_{t}\right)+\mathcal{O}\left(N^{-\eta}\right)$ for some $\eta>0$.

With the help of Lemma 6.5, Lemma 6.6 and Grönwall's Lemma, we obtain

Lemma 6.7. Let $W_{\beta} \in \mathcal{W}_{\beta}$. Let $\Psi_{t}$ the unique solution to $i \partial_{t} \Psi_{t}=H_{W_{\beta}} \Psi_{t}$ with initial datum $\Psi_{0} \in L_{s}^{2}\left(\mathbb{R}^{2 N}, \mathbb{C}\right) \cap H^{2}\left(\mathbb{R}^{2 N}, \mathbb{C}\right),\left\|\Psi_{0}\right\|=1$. Let $\varphi_{t}$ the unique solution to $i \partial_{t} \varphi_{t}=h_{b_{W_{\beta}}}^{G P} \varphi_{t}$ with $\varphi_{t} \in H^{3}\left(\mathbb{R}^{2}, \mathbb{C}\right),\left\|\varphi_{0}\right\|=1$.

(a) Let $\beta<1 / 12$ and $\alpha^{<}\left(\Psi_{t}, \varphi_{t}\right)$ be defined as in Definition 6.1 with $\xi=1 / 6$. Then,

$$
\alpha^{<}\left(\Psi_{t}, \varphi_{t}\right) \leq e^{\int_{0}^{t} d s \mathcal{K}\left(\varphi_{s}, A_{s}\right)}\left(\alpha^{<}\left(\Psi_{0}, \varphi_{0}\right)+N^{-2 \beta} \ln (N)\right) .
$$

(b) Let $\beta \geq 1 / 12$ and $\alpha^{<}\left(\Psi_{t}, \varphi_{t}\right)$ be defined as in Definition 6.1 with $\xi=1 / 10$. Then,

$$
\alpha^{<}\left(\Psi_{t}, \varphi_{t}\right) \leq e^{\int_{0}^{t} d s \mathcal{K}\left(\varphi_{s}, A_{s}\right)}\left(\alpha^{<}\left(\Psi_{0}, \varphi_{0}\right)+N^{-1 / 10}\right) .
$$

Proof. From Lemmas 6.5 and 6.6, we have

$$
\alpha^{<}\left(\Psi_{t}, \varphi_{t}\right) \leq \alpha^{<}\left(\Psi_{0}, \varphi_{0}\right)+\int_{0}^{t} d s \mathcal{K}\left(\varphi_{s}, A_{s}\right)\left(\alpha^{<}\left(\Psi_{s}, \varphi_{s}\right)+N^{-2 \beta} \ln (N)\right)
$$

in the case of $\beta<1 / 12$. Thus if we apply Grönwall's Lemma, we get

$$
\begin{aligned}
\alpha<\left(\Psi_{s}, \varphi_{s}\right) \leq & \alpha^{<}\left(\Psi_{0}, \varphi_{0}\right)+\int_{0}^{t} d s \mathcal{K}\left(\varphi_{s}, A_{s}\right) N^{-2 \beta} \ln (N) \\
& +\int_{0}^{t} d s \mathcal{K}\left(\varphi_{s}, A_{s}\right) e^{\int_{s}^{t} d \tau \mathcal{K}\left(\varphi_{\tau}, A_{\tau}\right)}\left(\alpha<\left(\Psi_{0}, \varphi_{0}\right)\right. \\
& \left.+\int_{0}^{s} d u \mathcal{K}\left(\varphi_{u}, A_{u}\right) N^{-2 \beta} \ln (N)\right)
\end{aligned}
$$

With the help of the relation $|x| \leq e^{|x|}$ this can be further simplified and one obtains (50). Part (b) of the Lemma is shown in complete analogy. 
Proof of Theorem 2.4: Part (a). Note that under the assumptions $\varphi_{t} \in H^{3}\left(\mathbb{R}^{2}, \mathbb{C}\right)$ and A. $\in C^{1}\left(\mathbb{R}, L^{\infty}\left(\mathbb{R}^{2}, \mathbb{R}\right)\right)$ there exists a constant $C_{t}<\infty$, depending on $t, \varphi_{0}$ and $A_{t}$, such that $\int_{0}^{t} d s \mathcal{K}\left(\varphi_{s}, A_{s}\right) \leq C_{t}$, see Sect. 4. Let $\beta<1 / 12$ and $\xi=1 / 6$. We now combine Lemmas 6.3 and 6.7 to estimate

$$
\begin{aligned}
\left.\operatorname{Tr}\left|\gamma_{\Psi_{t}}^{(1)}-\right| \varphi_{t}\right\rangle\left\langle\varphi_{t}|| \leq\right. & C \sqrt{\alpha^{<}\left(\Psi_{t}, \varphi_{t}\right)} \leq e^{C_{t}} \sqrt{\alpha^{<}\left(\Psi_{0}, \varphi_{0}\right)+N^{-2 \beta} \ln (N)} \\
\leq & e^{C_{t}}\left(\sqrt[4]{\left.\operatorname{Tr}\left|\gamma_{\Psi_{0}}^{(1)}-\right| \varphi_{0}\right\rangle\left\langle\varphi_{0}||\right.}+\sqrt{\left|\mathcal{E}_{W_{\beta}}\left(\Psi_{0}\right)-\mathcal{E}_{b_{W_{\beta}}}^{G P}\left(\varphi_{0}\right)\right|}\right. \\
& \left.+N^{-\beta} \sqrt{\ln (N)}\right) .
\end{aligned}
$$

Here, we have used $N^{-1 / 6} \leq N^{-2 \beta} \ln (N)$ and $\sqrt{|a|+|b|} \leq \sqrt{|a|}+\sqrt{|b|}$ to obtain the last line. In a similar way, one shows

$$
\begin{aligned}
\left|\mathcal{E}_{W_{\beta}}\left(\Psi_{t}\right)-\mathcal{E}_{b_{W_{\beta}}}^{G P}\left(\varphi_{t}\right)\right| \leq & \alpha^{<}\left(\Psi_{t}, \varphi_{t}\right) \\
\leq & e^{C_{t}}\left(\sqrt{\left.\operatorname{Tr}\left|\gamma_{\Psi_{0}}^{(1)}-\right| \varphi_{0}\right\rangle\left\langle\varphi_{0}||\right.}+\left|\mathcal{E}_{W_{\beta}}\left(\Psi_{0}\right)-\mathcal{E}_{b_{W_{\beta}}}^{G P}\left(\varphi_{0}\right)\right|\right. \\
& \left.+N^{-2 \beta} \ln (N)\right) .
\end{aligned}
$$

In total, this shows part (a) of Theorem 2.4 for $\beta<1 / 12$. The estimates for $\beta \geq 1 / 12$ are shown in exactly the same manner.

\subsection{Prooffor the exponential scaling $V_{N}$.}

6.2.1. Definition of the functional In case of the exponential scaling, the interaction is so strong such that the many-body wave function develops a non-negligible short scale correlation structure which prevents the particles from being localized too close to each other. These correlations determine the statical and dynamical properties of the condensate in a crucial manner and need to be taken into account explicitly. It is therefore reasonable to expect that the counting measure needs to be modified, too.

In order to motivate how the correlation structure will appear in the definition of the functional we think for the moment of the most simple counting measure, namely $\left\langle\left\langle\Psi_{t}, q_{1}^{\varphi_{t}} \Psi_{t}\right\rangle\right\rangle=1-\left\langle\left\langle\Psi_{t}, p_{1}^{\varphi_{t}} \Psi_{t}\right\rangle\right\rangle$. This functional counts the relative number of particles which are not in the state $\varphi_{t}$ and consequently measures if the many-body state is approximately given by the product state $\varphi_{t}^{\otimes N}$, in the sense of reduced density matrices. However, in the face of the exponential scaling, one should picture the many-body state not as the product of one-particle states but rather as a wave function of Jastrow-type, i.e.

$$
\begin{aligned}
\Psi_{t}\left(x_{1}, \ldots, x_{N}\right) & \approx \prod_{1 \leq i<j \leq N} j_{N, R}\left(x_{i}-x_{j}\right) \prod_{k=1}^{N} \varphi_{t}\left(x_{k}\right) \\
& =\prod_{l=2}^{N} j_{N, R}\left(x_{1}-x_{l}\right) \varphi_{t}\left(x_{1}\right)\left(\prod_{2 \leq i<j \leq N} j_{N, R}\left(x_{i}-x_{j}\right) \prod_{k=1}^{N} \varphi_{t}\left(x_{k}\right)\right)
\end{aligned}
$$


with $j_{N, R}$ being the zero energy scattering state as defined in (26).

In the following, we will consider the correlation structure to be induced by $f_{\mu}$ (see Definition 5.3) rather than by $j_{N, R}$. This replacement does not change the heuristic discussion above, since $f_{\mu}(x) \approx j_{N, R_{\mu}}(x) \forall|x| \leq N^{-\mu}$ for $N$ large (see Lemma 5.5), but will allow us to smoothen the singular interaction, as we will explain in the following.

Instead of projecting onto the state $\varphi_{t}$, the previous discussion suggests to replace $p_{1}^{\varphi_{t}}$ by $\left|\prod_{k=2}^{N} f_{\mu}\left(x_{1}-x_{k}\right) \varphi_{t}\left(x_{1}\right)\right\rangle\left\langle\prod_{l=2}^{N} f_{\mu}\left(x_{1}-x_{l}\right) \varphi_{t}\left(x_{1}\right)\right|$. The counting measure would then be given by

$$
\begin{aligned}
1 & -\left\langle\left\langle\Psi_{t}, \mid \prod_{k=2}^{N} f_{\mu}\left(x_{1}-x_{k}\right) \varphi_{t}\left(x_{1}\right)\right\rangle\left\langle\prod_{l=2}^{N} f_{\mu}\left(x_{1}-x_{l}\right) \varphi_{t}\left(x_{1}\right) \mid \Psi_{t}\right\rangle\right\rangle \\
& =1-\left\langle\left\langle\Psi_{t}, \prod_{k=2}^{N} f_{\mu}\left(x_{1}-x_{k}\right) p_{1}^{\varphi_{t}} \prod_{l=2}^{N} f_{\mu}\left(x_{1}-x_{l}\right) \Psi_{t}\right\rangle\right\rangle .
\end{aligned}
$$

This expression can be further simplified, if we use $g_{\mu}=1-f_{\mu}$ and only keep the terms which are at most linear in $g_{\mu}$

$$
\begin{aligned}
1 & \left.-\left\langle\Psi_{t},\left(1-\sum_{k=2}^{N} g_{\mu}\left(x_{1}-x_{k}\right)\right) p_{1}^{\varphi_{t}}\left(1-\sum_{l=2}^{N} g_{\mu}\left(x_{1}-x_{l}\right)\right) \Psi_{t}\right\rangle\right\rangle \\
& \approx 1-\left\langle\left\langle\Psi_{t}, p_{1}^{\varphi_{t}} \Psi_{t}\right\rangle+2(N-1) \Re\left\langle\left\langle\Psi_{t}, g_{\mu}\left(x_{1}-x_{2}\right) p_{1}^{\varphi} \Psi_{t}\right\rangle\right\rangle\right. \\
& =\left\langle\left\langle\Psi_{t}, q_{1}^{\varphi_{t}} \Psi_{t}\right\rangle+2(N-1) \Re\left\langle\left\langle\Psi_{t}, g_{\mu}\left(x_{1}-x_{2}\right) p_{1}^{\varphi} \Psi_{t}\right\rangle .\right.\right.
\end{aligned}
$$

With the help of the symmetry of the many-body wave function and the identity $q_{1}^{\varphi_{t}}=$ $1-p_{1}^{\varphi_{t}}$, we compute

$$
\frac{d}{d t}\left\langle\left\langle\Psi_{t}, q_{1}^{\varphi_{t}} \Psi_{t}\right\rangle=2 \Im\left(\left\langle\Psi_{t},\left((N-1) V_{N}\left(x_{1}-x_{2}\right)-4 \pi\left|\varphi_{t}\left(x_{1}\right)\right|^{2}\right) p_{1}^{\varphi_{t}} \Psi_{t}\right\rangle\right) .\right.
$$

Defining $h_{4 \pi}^{G P}\left(x_{1}\right)=\left(-\Delta_{1}+A_{t}\left(x_{1}\right)\right)+4 \pi\left|\varphi_{t}\left(x_{1}\right)\right|^{2}$, we further compute

$$
\begin{aligned}
& \frac{d}{d t} 2(N-1) \Re\left(\left\langle\Psi_{t}, g_{\mu}\left(x_{1}-x_{2}\right) p_{1}^{\varphi_{t}} \Psi_{t}\right\rangle\right) \\
& \quad=-2(N-1) \Im\left(\left\langle\Psi_{t},\left[H_{V_{N}}, g_{\mu}\left(x_{1}-x_{2}\right)\right] p_{1}^{\varphi_{t}} \Psi_{t}\right\rangle\right) \\
& \quad-2(N-1) \Im\left(\left\langle\Psi_{t}, g_{\mu}\left(x_{1}-x_{2}\right)\left[\left(H_{V_{N}}-h_{4 \pi}^{G P}\left(x_{1}\right)\right), p_{1}^{\varphi_{t}}\right] \Psi_{t}\right\rangle\right) .
\end{aligned}
$$

Using (30) and neglecting the mixed derivatives we get that $\left[H_{V_{N}}, g_{\mu}\left(x_{1}-x_{2}\right)\right] \approx$ $\left(V_{N}-M_{\mu}\right)\left(x_{1}-x_{2}\right) f_{\mu}\left(x_{1}-x_{2}\right)$. Further one can show that the leading order of $g_{\mu}\left(x_{1}-\right.$ $\left.x_{2}\right)\left[\left(H_{V_{N}}-h_{4 \pi}^{G P}\left(x_{1}\right)\right), p_{1}^{\varphi_{t}}\right]$ is given by $g_{\mu}\left(x_{1}-x_{2}\right) V_{N}\left(x_{1}-x_{2}\right) p_{1}^{\varphi_{t}}$. This is due to the smallness of the support of $g_{\mu}$ and $V_{N}$ which significantly overlap only for this term.

Hence the leading order of $\frac{d}{d t}(52)$ is given by

$$
\left.2 \Im\left(《\left((N-1) M_{\mu}\left(x_{1}-x_{2}\right) f_{\mu}\left(x_{1}-x_{2}\right)-4 \pi\left|\varphi_{t}\left(x_{1}\right)\right|^{2}\right) p_{1}^{\varphi_{t}} \Psi_{t}\right\rangle\right) .
$$

Summarizing we can say that due to this adjustment and by means of the scattering equation (30), the interaction $V_{N}$ got replaced by the less singular potential $M_{\mu} f_{\mu}$ in in 
the first line of the equation above. It is this less singular potential that can be controlled using the results from the previous chapter. $M_{\mu} f_{\mu}$ has the properties of the $W_{\beta}$ considered above [see Lemma $5.5(\mathrm{k})$ ], making (53) controllable. This explains why we chose to use $f_{\mu}$ in the definition of the modified counting functional instead of $j_{N, R}$. In return we obtain additional error terms, which, however, can be estimated sufficiently well, see Lemma 6.13.

Making use of Lemma 4.2 (c) and (d) this idea can also be used for weight functions different from $\frac{k}{N}$. Note that due to symmetry the correction term in (52) can be written as

$$
\begin{aligned}
& 2(N-1) \Re\left\langle\left\langle\Psi_{t}, g_{\mu}\left(x_{1}-x_{2}\right) p_{1}^{\varphi} \Psi_{t}\right\rangle\right. \\
& \quad=-N(N-1) \Re\left\langle\left\langle\Psi_{t}, g_{\mu}\left(x_{1}-x_{2}\right)\left(-N^{-1}\right)\left(p_{1}^{\varphi} q_{2}^{\varphi}+q_{1}^{\varphi} p_{2}^{\varphi}\right) \Psi_{t}\right\rangle\right\rangle \\
& \quad-N(N-1) \Re\left\langle\left\langle\Psi_{t}, g_{\mu}\left(x_{1}-x_{2}\right)\left(-2 N^{-1}\right) p_{1}^{\varphi} p_{2}^{\varphi} \Psi_{t}\right\rangle .\right.
\end{aligned}
$$

Moreover, $N^{-1}$ can be viewed as the discrete time derivative of the weight $\frac{k}{N}$, in other words

$$
\begin{aligned}
-N^{-1} & =\frac{k}{N}-\frac{k+1}{N}=n^{2}(k)-n^{2}(k+1) \text { and } \\
-2 N^{-1} & =\frac{k}{N}-\frac{k+2}{N}=n^{2}(k)-n^{2}(k+2) .
\end{aligned}
$$

We will use this insight to modify the functional $\alpha^{<}\left(\Psi_{t}, \varphi_{t}\right)$ from Definition 6.1. We first compute the time derivative of $\left\langle\left\langle\Psi_{t}, \widehat{m}^{\varphi_{t}} \Psi_{t}\right\rangle\right\rangle$ and then add an additional term to the counting measure in a way such that the interaction $V_{N}$ gets replaced by the potential $M_{\mu} f_{\mu}$.

Pursuing this approach results in the following definition.

Definition 6.8. Let $0<\xi<\frac{1}{3}, \mu>0$ and $m(k)$ be defined as in Definition 6.1. Moreover, let

$$
\begin{aligned}
m^{a}(k) & =m(k)-m(k+1), \\
m^{b}(k) & =m(k)-m(k+2) \text { and } \\
\widehat{r} & =\widehat{m}^{b} p_{1} p_{2}+\widehat{m}^{a}\left(p_{1} q_{2}+q_{1} p_{2}\right) .
\end{aligned}
$$

Then, $\alpha: L^{2}\left(\mathbb{R}^{2 N}, \mathbb{C}\right) \times L^{2}\left(\mathbb{R}^{2}, \mathbb{C}\right) \rightarrow \mathbb{R}_{0}^{+}$is defined by

$$
\alpha(\Psi, \varphi)=\left\langle\langle\Psi, \widehat{m} \Psi\rangle+\left|\mathcal{E}_{V_{N}}(\Psi)-\mathcal{E}_{4 \pi}^{G P}(\varphi)\right|-N(N-1) \Re\left(\left\langle\Psi, g_{\mu}\left(x_{1}-x_{2}\right) \widehat{r} \Psi\right\rangle\right) .\right.
$$

Remark 6.9. It should be noted that $\widehat{r}$ depends on $\xi$ and the functional $\alpha$ depends on $\xi$ and $\mu$. Both parameters are later chosen in a way such that we can establish an integral type Grönwall estimate.

If one recalls Definition 6.3, one sees that $\alpha$ is obtained from $\alpha<$ by adding an additional correction term. It is important to note that (see proof of Lemma 6.10)

$$
N(N-1) \mid \Re\left(\left|\Psi, g_{\mu}\left(x_{1}-x_{2}\right) \widehat{r} \Psi\right\rangle\right) \mid \leq C\|\varphi\|_{\infty} N^{-\mu+\xi} \ln (N) .
$$

For $\mu$ chosen large enough this allows us to show that the convergence of $\alpha$ to zero can be related to the notion of complete Bose-Einstein condensation in terms of reduced density matrices. 
Lemma 6.10. Let $0<\xi<1 / 3, \mu>0, \Psi \in L_{s}^{2}\left(\mathbb{R}^{2 N}, \mathbb{C}\right), \varphi \in L^{2}\left(\mathbb{R}^{2}, \mathbb{C}\right) \cap L^{\infty}\left(\mathbb{R}^{2}, \mathbb{C}\right)$ and $\alpha(\Psi, \varphi)$ be defined as in Definition 6.1. Then, there exists a constant $C \in(0, \infty)$ such that

$$
\begin{aligned}
\left.\operatorname{Tr}\left|\gamma_{\Psi}^{(1)}-\right| \varphi\right\rangle\langle\varphi|| \leq & \sqrt{8 \alpha(\Psi, \varphi)}+C\|\varphi\|_{\infty} N^{-1 / 2(\mu-\xi)} \sqrt{\ln (N)} \\
\alpha(\Psi, \varphi) \leq & \sqrt{\left.\operatorname{Tr}\left|\gamma_{\Psi}^{(1)}-\right| \varphi\right\rangle\langle\varphi||}+\left|\mathcal{E}_{V_{N}}(\Psi)-\mathcal{E}_{4 \pi}^{G P}(\varphi)\right| \\
& +\frac{1}{2} N^{-\xi}+C\|\varphi\|_{\infty} N^{-\mu+\xi} \ln (N)
\end{aligned}
$$

Proof. Using $\left\|\widehat{m}^{a}\right\|_{\mathrm{op}}+\left\|\widehat{m}^{b}\right\|_{\mathrm{op}} \leq C N^{-1+\xi}$, see (76), together with Eq. (16) and Lemma 5.5 (i), we obtain

$$
\begin{aligned}
\left\|g_{\mu}\left(x_{1}-x_{2}\right) \widehat{r}\right\|_{\mathrm{op}} & \leq\left\|g_{\mu}\left(x_{1}-x_{2}\right) p_{1}\left(\widehat{m}^{b} p_{2}+\widehat{m}^{a} q_{2}\right)\right\|_{\mathrm{op}}+\left\|g_{\mu}\left(x_{1}-x_{2}\right) p_{2} q_{1} \widehat{m}^{a}\right\|_{\mathrm{op}} \\
& \leq\|\varphi\|_{\infty}\left\|g_{\mu}\right\|\left(\left\|\widehat{m}^{a}\right\|_{\mathrm{op}}+\left\|\widehat{m}^{b}\right\|_{\mathrm{op}}\right) \\
& \leq\|\varphi\|_{\infty} N^{\xi-2-\mu} \ln (N) .
\end{aligned}
$$

Therefore, we bound $N(N-1)\left|\Re\left(\left\langle\Psi, g_{\mu}\left(x_{1}-x_{2}\right) \widehat{r} \Psi\right\rangle\right)\right| \leq\|\varphi\|_{\infty} N^{-\mu+\xi} \ln (N)$. By means of Lemma 6.3 the Lemma follows.

\subsubsection{Preliminaries for the Grönwall estimate}

Definition 6.11. Let $0<\xi<1 / 3, \mu>0$ and $\widehat{r}$ be defined as in Definition 6.8. Then, $\gamma: L^{2}\left(\mathbb{R}^{2 N}, \mathbb{C}\right) \times L^{2}\left(\mathbb{R}^{2}, \mathbb{C}\right) \rightarrow \mathbb{R}$ is defined by

$\gamma(\Psi, \varphi)=\left|\gamma_{a}(\Psi, \varphi)\right|+\left|\gamma_{b}(\Psi, \varphi)\right|+\left|\gamma_{c}(\Psi, \varphi)\right|+\left|\gamma_{d}(\Psi, \varphi)\right|+\left|\gamma_{e}(\Psi, \varphi)\right|+\left|\gamma_{f}(\Psi, \varphi)\right|$,

where the different summands are:

(a) The change in the energy-difference

$$
\gamma_{a}(\Psi, \varphi)=\left\langle\Psi \Psi, \dot{A}_{t}\left(x_{1}\right) \Psi\right\rangle-\left\langle\varphi, \dot{A}_{t} \varphi\right\rangle .
$$

(b) The new interaction term

$$
\begin{aligned}
\gamma_{b}(\Psi, \varphi)= & -N(N-1) \mathfrak{s}\left(\left\langle\Psi, \widetilde{Z}_{\mu}^{\varphi}\left(x_{1}, x_{2}\right) \widehat{r} \Psi\right\rangle\right) \\
& -N(N-1) \mathfrak{s}\left(\left\langle\Psi, g_{\mu}\left(x_{1}-x_{2}\right) \widehat{r} \mathcal{Z}^{\varphi}\left(x_{1}, x_{2}\right) \Psi\right\rangle\right),
\end{aligned}
$$

where, using $M_{\mu}$ from Definition 5.3,

$$
\begin{aligned}
& \widetilde{Z}_{\mu}^{\varphi}\left(x_{1}, x_{2}\right)=\left(M_{\mu}\left(x_{1}-x_{2}\right)-4 \pi \frac{|\varphi|^{2}\left(x_{1}\right)+|\varphi|^{2}\left(x_{2}\right)}{N-1}\right) f_{\mu}\left(x_{1}-x_{2}\right) \\
& \mathcal{Z}^{\varphi}\left(x_{1}, x_{2}\right)=V_{N}\left(x_{1}-x_{2}\right)-\frac{4 \pi}{N-1}|\varphi|^{2}\left(x_{1}\right)-\frac{4 \pi}{N-1}|\varphi|^{2}\left(x_{2}\right) .
\end{aligned}
$$

(c) The mixed derivative term

$$
\left.\gamma_{c}(\Psi, \varphi)=-4 N(N-1)\left\langle\Psi \Psi,\left(\nabla_{1} g_{\mu}\left(x_{1}-x_{2}\right)\right) \nabla_{1} \widehat{r} \Psi\right\rangle\right\rangle .
$$


(d) Three particle interactions

$$
\begin{aligned}
\gamma_{d}(\Psi, \varphi)= & 2 N(N-1)(N-2) \mathfrak{\Im}\left(\left\langle\Psi, g_{\mu}\left(x_{1}-x_{2}\right)\left[V_{N}\left(x_{1}-x_{3}\right), \widehat{r}\right] \Psi\right\rangle\right) \\
& -N(N-1)(N-2) \mathfrak{s}\left(\left\langle\Psi \Psi, g_{\mu}\left(x_{1}-x_{2}\right)\left[4 \pi|\varphi|^{2}\left(x_{3}\right), \widehat{r}\right] \Psi\right\rangle\right) .
\end{aligned}
$$

(e) Interaction terms of the correction

$$
\gamma_{e}(\Psi, \varphi)=\frac{1}{2} N(N-1)(N-2)(N-3) \Im\left(\left\langle\Psi, g_{\mu}\left(x_{1}-x_{2}\right)\left[V_{N}\left(x_{3}-x_{4}\right), \widehat{r}\right] \Psi\right\rangle\right) .
$$

(f) Correction terms of the mean field

$$
\gamma_{f}(\Psi, \varphi)=-2 N(N-2) \Im\left(\left\langle\Psi, g_{\mu}\left(x_{1}-x_{2}\right)\left[4 \pi|\varphi|^{2}\left(x_{1}\right), \widehat{r}\right] \Psi\right\rangle\right) .
$$

The value of $\alpha\left(\Psi_{t}, \varphi_{t}\right)$ at time $t$ is then bounded by

Lemma 6.12. Let $V_{N} \in \mathcal{V}_{N}$ and let $\Psi_{t}$ the unique solution to $i \partial_{t} \Psi_{t}=H_{V_{N}} \Psi_{t}$ with initial datum $\Psi_{0} \in L_{s}^{2}\left(\mathbb{R}^{2 N}, \mathbb{C}\right) \cap H^{2}\left(\mathbb{R}^{2 N}, \mathbb{C}\right),\left\|\Psi_{0}\right\|=1$. Let $\varphi_{t}$ the unique solution to $i \partial_{t} \varphi_{t}=h_{4 \pi}^{G P} \varphi_{t}$ with $\varphi_{t} \in H^{3}\left(\mathbb{R}^{2}, \mathbb{C}\right),\left\|\varphi_{0}\right\|=1$. Let $\alpha\left(\Psi_{t}, \varphi_{t}\right)$ and $\gamma\left(\Psi_{t}, \varphi_{t}\right)$ be defined as in (57) and (61). Then

$$
\alpha\left(\Psi_{t}, \varphi_{t}\right) \leq \alpha\left(\Psi_{0}, \varphi_{0}\right)+\int_{0}^{t} d s \gamma\left(\Psi_{s}, \varphi_{s}\right)
$$

Proof. We first calculate

$$
\begin{aligned}
\frac{\mathrm{d}}{\mathrm{d} t}\left(\langle\Psi, \widehat{m} \Psi\rangle-N(N-1) \Re\left(\left\langle\Psi, g_{\mu}\left(x_{1}-x_{2}\right) \widehat{r} \Psi\right\rangle\right)\right) \\
=-N(N-1) \mathfrak{\Im}\left(\left\langle\Psi_{t}, \mathcal{Z}^{\varphi_{t}}\left(x_{1}, x_{2}\right) \widehat{r} \Psi_{t}\right\rangle\right) \\
\quad-N(N-1) \Re\left(i\left\langle\left\langle\Psi_{t}, g_{\mu}\left(x_{1}-x_{2}\right)\left[H_{V_{N}}-\sum_{i=1}^{N} h_{4 \pi, i}^{G P}, \widehat{r}\right] \Psi_{t}\right\rangle\right)\right. \\
\quad-N(N-1) \Re\left(i\left\langle\Psi_{t},\left[H_{V_{N}}, g_{\mu}\left(x_{1}-x_{2}\right)\right] \widehat{r} \Psi_{t}\right\rangle\right) .
\end{aligned}
$$

Using symmetry and $\Re(i z)=-\Im(z)$, we obtain

$$
\begin{aligned}
\frac{\mathrm{d}}{\mathrm{d} t}\left(\langle\Psi, \widehat{m} \Psi\rangle-N(N-1) \Re\left(\left\langle\Psi, g_{\mu}\left(x_{1}-x_{2}\right) \widehat{r} \Psi\right\rangle\right)\right) \\
=-N(N-1) \mathfrak{s}\left(\left\langle\Psi_{t}, \mathcal{Z}^{\varphi_{t}}\left(x_{1}, x_{2}\right) \widehat{r} \Psi_{t}\right\rangle\right) \\
\quad+N(N-1) \mathfrak{s}\left(\left\langle\Psi_{t}, g_{\mu}\left(x_{1}-x_{2}\right)\left[\mathcal{Z}^{\varphi_{t}}\left(x_{1}, x_{2}\right), \widehat{r}\right] \Psi_{t}\right\rangle\right) \\
\quad+2 N(N-1)(N-2) \mathfrak{s}\left(\left\langle\Psi_{t}, g_{\mu}\left(x_{1}-x_{2}\right)\left[V_{N}\left(x_{1}-x_{3}\right), \widehat{r}\right] \Psi_{t}\right\rangle\right) \\
\quad-N(N-1)(N-2) \mathfrak{s}\left(\left\langle\Psi_{t}, g_{\mu}\left(x_{1}-x_{2}\right)\left[4 \pi\left|\varphi_{t}\right|^{2}\left(x_{3}\right), \widehat{r}\right] \Psi_{t}\right\rangle\right) \\
\quad+\frac{1}{2} N(N-1)(N-2)(N-3) \mathfrak{s}\left(\left\langle\Psi_{t}, g_{\mu}\left(x_{1}-x_{2}\right)\left[V_{N}\left(x_{3}-x_{4}\right), \widehat{r}\right] \Psi_{t}\right\rangle\right) \\
\quad+N(N-1) \mathfrak{s}\left(\left\langle\Psi_{t},\left[H_{V_{N}}, g_{\mu}\left(x_{1}-x_{2}\right)\right] \widehat{r} \Psi_{t}\right\rangle\right) . \\
\quad-2 N(N-2) \mathfrak{s}\left(\left\langle\left\langle\Psi_{t}, g_{\mu}\left(x_{1}-x_{2}\right)\left[4 \pi\left|\varphi_{t}\right|^{2}\left(x_{1}\right), \widehat{r}\right] \Psi_{t}\right\rangle\right) .\right.
\end{aligned}
$$


The third and fourth lines equal $\gamma_{d}$ (recall that $\Psi$ is symmetric), the fifth line equals $\gamma_{e}$ and the seventh line equals $\gamma_{f}$. Using that $\left(1-g_{\mu}\left(x_{1}-x_{2}\right)\right) \mathcal{Z}^{\varphi}\left(x_{1}, x_{2}\right)=\widetilde{Z}_{\mu}^{\varphi}\left(x_{1}, x_{2}\right)+$ $\left(V_{N}\left(x_{1}-x_{2}\right)-M_{\mu}\left(x_{1}-x_{2}\right)\right) f_{\mu}\left(x_{1}-x_{2}\right)$ we get

$$
\begin{aligned}
\frac{\mathrm{d}}{\mathrm{d} t}\left(\langle\Psi, \widehat{m} \Psi\rangle-N(N-1) \Re\left(\left\langle\Psi, g_{\mu}\left(x_{1}-x_{2}\right) \widehat{r} \Psi\right\rangle\right)\right) \\
\quad \leq \gamma_{d}\left(\Psi_{t}, \varphi_{t}\right)+\gamma_{e}\left(\Psi_{t}, \varphi_{t}\right)+\gamma_{f}\left(\Psi_{t}, \varphi_{t}\right) \\
\quad-N(N-1) \mathfrak{s}\left(\left\langle\Psi_{t}, \widetilde{Z}_{\mu}^{\varphi_{t}}\left(x_{1}, x_{2}\right) \widehat{r} \Psi_{t}\right\rangle\right) \\
\quad-N(N-1) \mathfrak{s}\left(\left\langle\Psi_{t},\left(V_{N}\left(x_{1}-x_{2}\right)-M_{\mu}\left(x_{1}-x_{2}\right)\right) f_{\mu}\left(x_{1}-x_{2}\right) \widehat{r} \Psi_{t}\right\rangle\right) \\
\quad-N(N-1) \Im\left(\left\langle\Psi_{t}, g_{\mu}\left(x_{1}-x_{2}\right) \widehat{r} \mathcal{Z}^{\varphi_{t}}\left(x_{1}, x_{2}\right) \Psi_{t}\right\rangle\right) \\
\quad+N(N-1) \Im\left(\left\langle\Psi_{t},\left[H_{V_{N}}, g_{\mu}\left(x_{1}-x_{2}\right)\right] \widehat{r} \Psi_{t}\right\rangle\right) .
\end{aligned}
$$

The first, second and the fourth line give $\gamma_{b}+\gamma_{d}+\gamma_{e}+\gamma_{f}$. Using Definition (5.3) the commutator in the fifth line equals

$$
\begin{aligned}
{\left[H_{V_{N}}, g_{\mu}\left(x_{1}-x_{2}\right)\right]=} & -\left[H_{V_{N}}, f_{\mu}\left(x_{1}-x_{2}\right)\right] \\
= & {\left[\Delta_{1}+\Delta_{2}, f_{\mu}\left(x_{1}-x_{2}\right)\right] } \\
= & \left(\Delta_{1}+\Delta_{2}\right) f_{\mu}\left(x_{1}-x_{2}\right) \\
& +\left(2 \nabla_{1} f_{\mu}\left(x_{1}-x_{2}\right)\right) \nabla_{1}+\left(2 \nabla_{2} f_{\mu}\left(x_{1}-x_{2}\right)\right) \nabla_{2} \\
= & \left(V_{N}\left(x_{1}-x_{2}\right)-M_{\mu}\left(x_{1}-x_{2}\right)\right) f_{\mu}\left(x_{1}-x_{2}\right) \\
& -\left(2 \nabla_{1} g_{\mu}\left(x_{1}-x_{2}\right)\right) \nabla_{1}-\left(2 \nabla_{2} g_{\mu}\left(x_{1}-x_{2}\right)\right) \nabla_{2} .
\end{aligned}
$$

Using symmetry the third and fifth line in (63) give

$$
-4 N(N-1)\left\langle\left\langle\Psi_{t},\left(\nabla_{1} g_{\mu}\left(x_{1}-x_{2}\right)\right) \nabla_{1} \widehat{r} \Psi_{t}\right\rangle=\gamma_{c}\left(\Psi_{t}, \varphi_{t}\right) .\right.
$$

By means of

$$
\frac{d}{d t}\left(\mathcal{E}_{M_{\mu}}\left(\Psi_{t}\right)-\mathcal{E}_{N\left\|M_{\mu}\right\|_{1}}^{G P}\left(\varphi_{t}\right)\right)=\gamma_{a}\left(\Psi_{t}, \varphi_{t}\right)
$$

and the fundamental theorem of calculus the result follows.

6.2.3. The Grönwall estimate Again, we will bound the time derivative of $\alpha\left(\Psi_{t}, \varphi_{t}\right)$ such that we can employ a Grönwall estimate.

Lemma 6.13. Let $V_{N} \in \mathcal{V}_{N}$. Let $\Psi_{t}$ the unique solution to $i \partial_{t} \Psi_{t}=H_{V_{N}} \Psi_{t}$ with initial datum $\Psi_{0} \in L_{s}^{2}\left(\mathbb{R}^{2 N}, \mathbb{C}\right) \cap H^{2}\left(\mathbb{R}^{2 N}, \mathbb{C}\right)$ and $\left\|\Psi_{0}\right\|=1$. Let $\varphi_{t}$ the unique solution to $i \partial_{t} \varphi_{t}=h_{4 \pi}^{G P} \varphi_{t}$ with $\varphi_{t} \in H^{3}\left(\mathbb{R}^{2}, \mathbb{C}\right)$ and $\left\|\varphi_{0}\right\|=1$. Let $\mathcal{E}_{V_{N}}\left(\Psi_{0}\right) \leq$ C. Let $\alpha\left(\Psi_{t}, \varphi_{t}\right)$, $\gamma_{i}\left(\Psi_{t}, \varphi_{t}\right), i \in\{a, b, c, d, e, f\}$ be defined as in Definitions 6.8 and 6.11 with $\xi=1 / 10$ and $\mu=10$. Then,

$$
\sum_{i \in\{a, b, c, d, e, f\}}\left|\gamma_{i}\left(\Psi_{t}, \varphi_{t}\right)\right| \leq \mathcal{K}\left(\varphi_{t}, A_{t}\right)\left(\alpha\left(\Psi_{t}, \varphi_{t}\right)+N^{-1 / 10}\right) .
$$

The proof of the Lemma can be found in Sect. 7.4. By means of Lemma 5.5 (h) and (i), the terms $\gamma_{a}$ and $\gamma_{b}$ can be estimated in the same way as $\gamma_{a}^{<}$and $\gamma_{b}{ }^{<}$. The estimates for $\gamma_{c}, \gamma_{d}, \gamma_{e}$ and $\gamma_{f}$ are based on the smallness of the $L^{p}$-norms of $g_{\mu}$, see Lemma 5.5 (i).

Thus, combining Lemmas 6.12 and 6.13, we obtain the following estimate for $\alpha\left(\Psi_{t}, \varphi_{t}\right)$ by means of Grönwall's Lemma 
Lemma 6.14. Let $V_{N} \in \mathcal{V}_{N}$. Let $\Psi_{t}$ the unique solution to $i \partial_{t} \Psi_{t}=H_{V_{N}} \Psi_{t}$ with initial datum $\Psi_{0} \in L_{S}^{2}\left(\mathbb{R}^{2 N}, \mathbb{C}\right) \cap H^{2}\left(\mathbb{R}^{2 N}, \mathbb{C}\right)$ and $\left\|\Psi_{0}\right\|=1$. Let $\varphi_{t}$ the unique solution to $i \partial_{t} \varphi_{t}=h_{4 \pi}^{G P} \varphi_{t}$ with $\varphi_{t} \in H^{3}\left(\mathbb{R}^{2}, \mathbb{C}\right)$. Let $\mathcal{E}_{V_{N}}\left(\Psi_{0}\right) \leq C$. Let $\alpha\left(\Psi_{t}, \varphi_{t}\right)$ be defined as in Definition 6.8 with $\xi=1 / 10$ and $\mu=10$. Then,

$$
\alpha\left(\Psi_{t}, \varphi_{t}\right) \leq e^{\int_{0}^{t} d s \mathcal{K}\left(\varphi_{s}, A_{s}\right)}\left(\alpha\left(\Psi_{0}, \varphi_{0}\right)+N^{-1 / 10}\right) .
$$

Proof. This is proven in the same way as Lemma 6.7.

Proof of Theorem 2.4: Part (b). Again, we note that under the assumptions $\varphi_{t} \in H^{3}\left(\mathbb{R}^{2}\right.$, $\mathbb{C})$ and $A . \in C^{1}\left(\mathbb{R}, L^{\infty}\left(\mathbb{R}^{2}, \mathbb{R}\right)\right)$ there exists a constant $C_{t}<\infty$, depending on $t, \varphi_{0}$ and $A_{t}$, such that $\int_{0}^{t} d s \mathcal{K}\left(\varphi_{s}, A_{s}\right) \leq C_{t}$, see Sect. 4 .

Let $\xi=1 / 10$ and $\mu=10$. If we then combine Lemmas 6.10 and 6.14 to estimate

$$
\begin{aligned}
\left.\operatorname{Tr}\left|\gamma_{\Psi_{t}}^{(1)}-\right| \varphi_{t}\right\rangle\left\langle\varphi_{t}||\right. & \leq C \sqrt{\alpha\left(\Psi_{t}, \varphi_{t}\right)}+C\left\|\varphi_{t}\right\|_{\infty} N^{-1} \leq e^{C_{t}}\left(\sqrt{\alpha\left(\Psi_{0}, \varphi_{0}\right)}+N^{-1 / 20}\right) \\
& \leq e^{C_{t}}\left(\sqrt[4]{\left.\operatorname{Tr}\left|\gamma_{\Psi_{0}}^{(1)}-\right| \varphi_{0}\right\rangle\left\langle\varphi_{0}||\right.}+\sqrt{\left|\mathcal{E}_{V_{N}}\left(\Psi_{0}\right)-\mathcal{E}_{4 \pi}^{G P}\left(\varphi_{0}\right)\right|}+N^{-1 / 20}\right) .
\end{aligned}
$$

Moreover, one obtains

$$
\begin{aligned}
\left|\mathcal{E}_{V_{N}}\left(\Psi_{t}\right)-\mathcal{E}_{4 \pi}^{G P}\left(\varphi_{t}\right)\right| & \leq \alpha\left(\Psi_{t}, \varphi_{t}\right)+C\left\|\varphi_{t}\right\|_{\infty} N^{-1} \\
& \leq e^{C_{t}}\left(\sqrt{\left.\operatorname{Tr}\left|\gamma_{\Psi_{0}}^{(1)}-\right| \varphi_{0}\right\rangle\left\langle\varphi_{0}||\right.}+\left|\mathcal{E}_{V_{N}}\left(\Psi_{0}\right)-\mathcal{E}_{4 \pi}^{G P}\left(\varphi_{0}\right)\right|+N^{-1 / 10}\right) .
\end{aligned}
$$

Finally, this shows part (b) of Theorem 2.4.

\section{Rigorous Estimates}

7.1. Smearing out the potential $W_{\beta}$. To control the potential $W_{\beta}$ for $\beta$ large, we use a technique which allows us to replace the potential $W_{\beta}$ by some potential $U_{\beta_{1}, \beta} \in$ $\mathcal{W}_{\beta_{1}}, \beta_{1}<\beta$ with $\left\|W_{\beta}\right\|_{1}=\left\|U_{\beta_{1}, \beta}\right\|_{1}$. For this, define $h_{\beta_{1}, \beta}$ by $\Delta h_{\beta_{1}, \beta}=W_{\beta}-U_{\beta_{1}, \beta}$. The function $h_{\beta_{1}, \beta}$ can be thought as an electrostatic potential which is caused by the charge $W_{\beta}-U_{\beta_{1}, \beta}$. It is then possible to rewrite

$$
\begin{aligned}
& \left\langle\chi \chi, W_{\beta}\left(x_{1}-x_{2}\right) \Omega\right\rangle=\left\langle\left\langle\chi, U_{\beta_{1}, \beta}\left(x_{1}-x_{2}\right) \Omega\right\rangle\right. \\
& \quad-\left\langle\left\langle\nabla_{1} \chi,\left(\nabla_{1} h_{\beta_{1}, \beta}\right)\left(x_{1}-x_{2}\right) \Omega\right\rangle-\left\langle\chi \chi,\left(\nabla_{1} h_{\beta_{1}, \beta}\right)\left(x_{1}-x_{2}\right) \nabla_{1} \Omega\right\rangle,\right.
\end{aligned}
$$

for $\chi, \omega \in L_{s}^{2}\left(\mathbb{R}^{2 N}, \mathbb{C}\right)$. We will verify that the $L^{p}$-norms of $h_{\beta_{1}, \beta}$ and $\nabla h_{\beta_{1}, \beta}$ are better to control than the respective $L^{p}$-norm of $W_{\beta}$. With additional control of $\nabla_{1} \Omega$ and $\nabla_{1} \chi$, it is therefore possible to obtain a sufficient bound for $\left\langle\chi, W_{\beta}\left(x_{1}-x_{2}\right) \Omega\right\rangle$ for large $\beta$.

Definition 7.1. For any $0 \leq \beta_{1}<\beta$ and any $W_{\beta} \in \mathcal{W}_{\beta}$ we define

$$
U_{\beta_{1}, \beta}(x)= \begin{cases}\frac{4}{\pi}\left\|W_{\beta}\right\|_{1} N^{2 \beta_{1}} & \text { for }|x|<1 / 2 N^{-\beta_{1}}, \\ 0 & \text { else. }\end{cases}
$$

and

$$
h_{\beta_{1}, \beta}(x)=\frac{1}{2 \pi} \int_{\mathbb{R}^{2}} \ln |x-y|\left(W_{\beta}(y)-U_{\beta_{1}, \beta}(y)\right) d^{2} y .
$$


Lemma 7.2. Let $0 \leq \beta_{1}<\beta, W_{\beta} \in \mathcal{W}_{\beta}$ and $N \in \mathbb{N}$ large enough such that $\operatorname{supp}\left(W_{\beta}\right) \subseteq \operatorname{supp}\left(U_{\beta_{1}, \beta}\right)$. Then,

(a)

$$
\begin{aligned}
& U_{\beta_{1}, \beta} \in \mathcal{W}_{\beta_{1}}, \\
& \Delta h_{\beta_{1}, \beta}=W_{\beta}-U_{\beta_{1}, \beta} .
\end{aligned}
$$

(b) Pointwise estimates

$$
\begin{aligned}
& h_{\beta_{1}, \beta}(x)=0 \text { for }|x| \geq 1 / 2 N^{-\beta_{1}}, \quad\left|h_{\beta_{1}, \beta}(x)\right| \leq C N^{-1} \ln (N), \\
&\left|\nabla h_{\beta_{1}, \beta}(x)\right| \leq C N^{-1}\left(|x|^{2}+N^{-2 \beta}\right)^{-\frac{1}{2}} .
\end{aligned}
$$

(c) Norm estimates

$$
\begin{aligned}
\left\|h_{\beta_{1}, \beta}\right\|_{\infty} & \leq C N^{-1} \ln (N), \\
\left\|h_{\beta_{1}, \beta}\right\|_{\lambda} & \leq C N^{-1-\frac{2}{\lambda} \beta_{1}} \ln (N) \text { for } 1 \leq \lambda \leq \infty, \\
\left\|\nabla h_{\beta_{1}, \beta}\right\|_{\lambda} & \leq C N^{-1+\beta-\frac{2}{\lambda} \beta_{1}} \quad \text { for } 1 \leq \lambda \leq \infty .
\end{aligned}
$$

Furthermore, for $\lambda=2$, we obtain the improved bounds

$$
\begin{aligned}
\left\|h_{0, \beta}\right\| & \leq C N^{-1}, \\
\left\|\nabla h_{\beta_{1}, \beta}\right\| & \leq C N^{-1}(\ln (N))^{1 / 2} .
\end{aligned}
$$

Proof. (a) $U_{\beta_{1}, \beta} \in \widetilde{\mathcal{W}}_{\beta_{1}}$ follows directly from the definition of $U_{\beta_{1}, \beta}$. Since $W_{\beta} \in$ $\mathcal{W}_{\beta}$ one has $|N|\left|U_{\beta_{1}, \beta} \|_{1}-b_{W_{\beta}}\right| \leq C N^{-1} \ln (N)$ and consequently $U_{\beta_{1}, \beta} \in \mathcal{W}_{\beta_{1}}$. Furthermore, $h_{\beta_{1}, \beta}$ is a solution of Poisson's equation because $-\frac{1}{2 \pi} \ln |x-y|$ is the radially symmetric Green's function of the Laplacian in two dimensions [36, Theorem 6.21].

(b) The first statement is a well known result from standard electrodynamics. It follows from Newton's theorem [36, Theorem 9.7] and $\left\|U_{\beta_{1}, \beta}\right\|_{1}=\left\|W_{\beta}\right\|_{1}$. Heuristically speaking, $W_{\beta}$ can be understood as a charge density and $-U_{\beta_{1}, \beta}$ as a smeared out charge density of opposite sign such that the "total charge" is zero. Moreover if we use that $W_{\beta}(x)=U_{\beta_{1}, \beta}(x)=0$ for all $|x| \geq 1 / 2 N^{-\beta_{1}}$, we obtain the pointwise estimate

$$
\begin{aligned}
\left|h_{\beta_{1}, \beta}(x)\right| \leq & \frac{1}{2 \pi} \int_{B_{1 / 2 N^{-\beta_{1}(0)}}} d^{2} y|\ln | x-y\left|W_{\beta}(y)\right| \\
& +\frac{1}{2 \pi} \int_{B_{1 / 2 N^{-\beta_{1}(0)}} d^{2} y|\ln | x-y\left|U_{\beta_{1}, \beta}(y)\right| .}
\end{aligned}
$$

Subsequently, we estimate each term separately. Therefore, it is useful to recall that there exists an $R \in(0, \infty)$ such that $W_{\beta}(x)=0$ for all $|x| \geq R N^{-\beta}$. This allows us to bound the first summand by

$$
\int_{B_{1 / 2 N^{-} \beta_{1}(0)}} d^{2} y|\ln | x-y|| W_{\beta}(y) \leq \int_{B_{R N^{-\beta}(0)}} d^{2} y|\ln | x-y|| W_{\beta}(y) .
$$


For $2 R N^{-\beta}<|x|<1 / 2 N^{-\beta_{1}}$ one has $|x-y| \leq N^{-\beta_{1}} \leq 1$ in the integral above. This implies $|\ln | x-y||=-\ln |x-y|$ and leads to

$$
\begin{aligned}
\int_{B_{1 / 2 N^{-} \beta_{1}(0)}} d^{2} y|\ln | x-y|| W_{\beta}(y) & \leq-\left\|W_{\beta}\right\|_{1} \ln \left(|x|-R N^{-\beta}\right) \leq-\left\|W_{\beta}\right\|_{1} \ln \left(R N^{-\beta}\right) \\
& \leq C\left\|W_{\beta}\right\|_{1} \ln N^{\beta} \leq C N^{-1} \ln (N)
\end{aligned}
$$

for all $2 R N^{-\beta}<|x|<1 / 2 N^{-\beta_{1}}$.

Let next $|x| \leq 2 R N^{-\beta}$. We again have $|x-y| \leq 1$ for all $y \in B_{1 / 2 N^{-\beta_{1}(0)}}$ and obtain

$$
\begin{aligned}
\int_{B_{1 / 2 N^{-\beta}(0)}|\ln | x-y|| W_{\beta}(y) d^{2} y} \leq C\left\|W_{\beta}\right\|_{\infty} \int_{B_{R N^{-\beta}(0)}}-\ln |x-y| d^{2} y \\
\leq C N^{-1+2 \beta} \int_{B_{R N^{-\beta}(x)}-\ln |y| d^{2} y} \\
\leq C N^{-1+2 \beta} \int_{B_{4 R N^{-\beta}(0)}-\ln |y| d^{2} y} \\
=C N^{-1+2 \beta}\left[-|y|^{2}(2 \ln |y|-1)\right]_{0}^{4 R N^{-\beta}} \\
\leq C N^{-1} \ln \left(N^{\beta}\right)
\end{aligned}
$$

for all $|x| \leq 2 R N^{-\beta}$. If we repeat the same estimate for $|x| \leq 1 / 2 N^{-\beta_{1}}$ and $U_{\beta_{1}, \beta}$ with $\left\|U_{\beta_{1}, \beta}\right\|_{\infty} \leq C N^{-1+2 \beta_{1}}$ we get

$$
\begin{aligned}
\int_{B_{1 / 2 N^{-} \beta_{1}(0)}|\ln | x-y|| U_{\beta_{1}, \beta}(y) d^{2} y} & \leq C\left\|U_{\beta_{1}, \beta}\right\|_{\infty} \int_{B_{1 / 2 N^{-\beta_{1}(0)}}}-\ln |x-y| d^{2} y \\
& \leq C N^{-1} \ln \left(N^{\beta_{1}}\right),
\end{aligned}
$$

which proves the first statement.

For the gradient, we estimate the two terms on the r.h.s. of

$$
\left|\nabla h_{\beta_{1}, \beta}(x)\right| \leq \frac{1}{2 \pi} \int \frac{1}{|x-y|} W_{\beta}(y) d^{2} y+\frac{1}{2 \pi} \int \frac{1}{|x-y|} U_{\beta_{1}, \beta}(y) d^{2} y
$$

separately. Let first $2 R N^{-\beta} \leq|x|$. Similarly as in the previous argument, one finds

$$
\int \frac{1}{|x-y|} W_{\beta}(y) d^{2} y \leq \int_{B_{R N^{-\beta}}(0)} \frac{1}{|x-y|} W_{\beta}(y) d^{2} y \leq \frac{\left\|W_{\beta}\right\|_{1}}{|x|-R N^{-\beta}}
$$

for $R N^{-\beta} \leq|x|$, which implies that

$$
\int \frac{1}{|x-y|} W_{\beta}(y) d^{2} y \leq \frac{C\left\|W_{\beta}\right\|_{1}}{\left(|x|^{2}+N^{-2 \beta}\right)^{\frac{1}{2}}} \leq \frac{C N^{-1}}{\left(|x|^{2}+N^{-2 \beta}\right)^{\frac{1}{2}}}
$$

for all $2 R N^{-\beta} \leq|x|$. For $|x| \leq 2 R N^{-\beta}$, we make use of

$$
N^{\beta} \leq \frac{C}{\left(|x|^{2}+N^{-2 \beta}\right)^{1 / 2}}
$$


and estimate

$$
\begin{aligned}
\int \frac{1}{|x-y|} W_{\beta}(y) d^{2} y & \leq\left\|W_{\beta}\right\|_{\infty} \int_{B_{R N^{-\beta}(0)}} \frac{1}{|x-y|} d^{2} y \\
& \leq C N^{2 \beta-1} \int_{0}^{R N^{-\beta}} d|y|=C N^{-1+\beta} \leq \frac{C N^{-1}}{\left(|x|^{2}+N^{-2 \beta}\right)^{1 / 2}} .
\end{aligned}
$$

Equivalently, we obtain

$$
\begin{aligned}
\int \frac{1}{|x-y|} U_{\beta_{1}, \beta}(y) d^{2} y & \leq\left\|U_{\beta_{1}, \beta}\right\|_{\infty} \int_{B_{N^{-} \beta_{1}(0)}} \frac{1}{|x-y|} d^{2} y \\
& =C N^{-1+\beta_{1}} \leq \frac{C N^{-1}}{\left(|x|^{2}+N^{-2 \beta_{1}}\right)^{1 / 2}} \leq \frac{C N^{-1}}{\left(|x|^{2}+N^{-2 \beta}\right)^{\frac{1}{2}}},
\end{aligned}
$$

for $|x| \leq N^{-\beta_{1}}$. Since $\nabla h_{\beta_{1}, \beta}(x)=0$ for $|x| \geq N^{-\beta_{1}}$, the second statement of (b) follows.

(c) The first part of (c) follows from (b) and the fact that the support of $h_{\beta 1, \beta}$ and $\nabla h_{\beta_{1}, \beta}$ has radius $\leq C N^{-\beta_{1}}$. The bounds on the $L^{2}$-norm can be improved by

$$
\begin{aligned}
\left\|\nabla h_{\beta_{1}, \beta}\right\|_{2}^{2} & \leq C \int_{0}^{C N^{-\beta_{1}}} d r r\left|\nabla h_{\beta_{1}, \beta}(r)\right|^{2} \leq \frac{C}{N^{2}} \int_{0}^{C N^{-\beta_{1}}} d r \frac{r}{r^{2}+N^{-2 \beta}} \\
& =\frac{C}{N^{2}} \ln \left(\frac{N^{-2 \beta_{1}}+N^{-2 \beta}}{N^{-2 \beta}}\right) \leq \frac{C}{N^{2}} \ln (N) .
\end{aligned}
$$

By means of [36, Theorem 9.7] we obtain

$$
\left|h_{0, \beta}(x)\right| \leq \frac{1}{2 \pi}|\ln (x)| \int\left(U_{0, \beta}(y)+W_{\beta}(y)\right) d^{2} y \leq C N^{-1}|\ln (x)|
$$

and

$$
\left\|h_{0, \beta}\right\|_{2}^{2} \leq C N^{-2} \int_{0}^{1} d r r \ln ^{2}(r) \leq C N^{-2},
$$

where we have used that $h_{0, \beta}(x)=0$ for all $|x| \geq 1$.

7.2. Estimates on the cutoff. In order to smear out singular potentials as explained in the previous section and to obtain sufficient bounds, it seems at first necessary to show that $\left\|\nabla_{1} q_{1} \Psi_{t}\right\|$ decays in $N$. However, this term will in fact not be small for the dynamic generated by $V_{N}$. There, we rather expect that $\left\|\nabla_{1} q_{1} \Psi_{t}\right\|=\mathcal{O}(1)$ holds. It has been shown in $[18,37]$ that the interaction energy is purely kinetic in the Gross-Pitaevskii regime, which implies that a relevant part of the kinetic energy is concentrated around the scattering centers. We must thus cutoff the part which is used to form the microscopic structure. For this, we define the set $\overline{\mathcal{A}}_{j}^{(d)}$ which includes all configurations where the distance between particle $x_{i}$ and particle $x_{j}, j \neq i$ is smaller than $N^{-d}$. It is then possible to prove that the kinetic energy concentrated on the complement of $\overline{\mathcal{A}}_{j}^{(d)}$, i.e. $\left\|\mathbb{1}_{\mathcal{A}_{1}^{(d)}} \nabla_{1} q_{1} \Psi\right\|$, is small, see Lemma 7.9. 
Definition 7.3. For any $j, k=1, \ldots, N$ and $d>0$ let

$$
\begin{aligned}
& a_{j, k}^{(d)}=\left\{\left(x_{1}, x_{2}, \ldots, x_{N}\right) \in \mathbb{R}^{2 N}:\left|x_{j}-x_{k}\right|<N^{-d}\right\} \subseteq \mathbb{R}^{2 N} \\
& \overline{\mathcal{A}}_{j}^{(d)}=\bigcup_{k \neq j} a_{j, k}^{(d)} \quad \mathcal{A}_{j}^{(d)}=\mathbb{R}^{2 N} \backslash \overline{\mathcal{A}}_{j}^{(d)} \quad \overline{\mathcal{B}}_{j}^{(d)}=\bigcup_{k \neq l \neq j} a_{k, l}^{(d)} \quad \mathcal{B}_{j}^{(d)}=\mathbb{R}^{2 N} \backslash \overline{\mathcal{B}}_{j}^{(d)} .
\end{aligned}
$$

Lemma 7.4. (a) For all $j \neq k$ with $1 \leq j, k \leq N$,

$$
\begin{aligned}
\left\|\mathbb{1}_{\overline{\mathcal{A}}_{j}^{(d)}} p_{j}\right\|_{o p} & \leq C\|\varphi\|_{\infty} N^{1 / 2-d}, \\
\left\|\mathbb{1}_{\overline{\mathcal{A}}_{j}^{(d)}} \nabla_{j} p_{j}\right\|_{o p} & \leq C\|\nabla \varphi\|_{\infty} N^{1 / 2-d}, \\
\left\|\mathbb{1}_{a_{j, k}^{(d)}} p_{j}\right\|_{o p} & \leq C\|\varphi\|_{\infty} N^{-d} .
\end{aligned}
$$

(b) Let $\Psi \in H^{1}\left(\mathbb{R}^{2 N}, \mathbb{C}\right)$. For any $1<p<\infty$, there exists a positive constant $C_{p}$, such that

$$
\left\|\mathbb{1}_{\overline{\mathcal{A}}_{1}^{(d)}} \Psi\right\|^{2} \leq C_{p} N^{(1-2 d) \frac{p-1}{p}}\left\|\nabla_{1} \Psi\right\|^{2 \frac{p-1}{p}}\|\Psi\|^{\frac{2}{p}}
$$

(c) Let $\Psi \in L_{s}^{2}\left(\mathbb{R}^{2 N}, \mathbb{C}\right) \cap H^{1}\left(\mathbb{R}^{2 N}, \mathbb{C}\right),\|\Psi\|_{H^{1}} \leq C$. For any $\epsilon>0$, there exists a positive constant $C_{\epsilon}$ such that

$$
\left\|\mathbb{1}_{\overline{\mathcal{B}}_{j}^{(d)}} \Psi\right\| \leq C_{\epsilon} N^{1-d+\epsilon} .
$$

(d) For any $k \neq j$

$$
\left\|\left[\mathbb{1}_{\mathcal{A}_{j}^{(d)}}, p_{k}\right]\right\|_{o p}=\left\|\left[\mathbb{1}_{a_{j, k}^{(d)}}, p_{k}\right]\right\|_{o p}=\left\|\left[\mathbb{1}_{\mathcal{A}_{j}^{(d)}}, p_{k}\right]\right\|_{o p} \leq C\|\varphi\|_{\infty} N^{-d}
$$

Proof. (a) First note that the volume of the sets $a_{j, k}^{(d)}$ introduced in Definition 7.3 are $\left|a_{j, k}^{(d)}\right|=\pi N^{-2 d}$

$$
\left\|\mathbb{1}_{\overline{\mathcal{A}}_{j}^{(d)}} p_{j}\right\|_{\mathrm{op}}=\left\|\mathbb{1}_{\overline{\mathcal{A}}_{1}^{(d)}} p_{1}\right\|_{\mathrm{op}}=\left\|p_{1} \mathbb{1}_{\overline{\mathcal{A}}_{1}^{(d)}} p_{1}\right\|_{\mathrm{op}}^{\frac{1}{2}} \leq\left(\|\varphi\|_{\infty}^{2}\left\|\mathbb{1}_{\overline{\mathcal{A}}_{1}^{(d)}}\right\|_{1, \infty}\right)^{1 / 2}
$$

where we defined

$$
\|f\|_{p, \infty}=\sup _{x_{2}, \ldots, x_{N} \in \mathbb{R}^{2}}\left(\int d x_{1}\left|f\left(x_{1}, \ldots, x_{N}\right)\right|^{p}\right)^{\frac{1}{p}} .
$$

Using $\mathbb{1}_{\overline{\mathcal{A}}_{1}^{(d)}} \leq \sum_{k=2}^{N} \mathbb{1}_{a_{1, k}^{(d)}}$ as well as $\left(\mathbb{1}_{\overline{\mathcal{A}}_{1}^{(d)}}\right)^{p}=\mathbb{1}_{\overline{\mathcal{A}}_{1}^{(d)}}$, we obtain

$$
\left\|\mathbb{1}_{\overline{\mathcal{A}}_{1}^{(d)}}\right\|_{p, \infty} \leq \sup _{x_{2}, \ldots, x_{N} \in \mathbb{R}^{2}}\left(\int d x_{1} \sum_{k=2}^{N} \mathbb{1}_{a_{1, k}^{(d)}}\right)^{\frac{1}{p}} \leq\left(N\left|a_{1, k}\right|\right)^{\frac{1}{p}} \leq C N^{(1-2 d) \frac{1}{p}} .
$$


This implies

$$
\left\|\mathbb{1}_{\overline{\mathcal{A}}_{j}^{(d)}} p_{j}\right\|_{\mathrm{op}} \leq C\|\varphi\|_{\infty} N^{\frac{1}{2}-d} .
$$

The second statement of (a) can be proven similarly. Analogously, we obtain

$$
\left\|\mathbb{1}_{a_{j, k}^{(d)}} p_{j}\right\|_{\mathrm{op}} \leq\|\varphi\|_{\infty}\left|a_{j, k}^{(d)}\right|^{1 / 2} \leq C\|\varphi\|_{\infty} N^{-d}
$$

(b) Without loss of generality, we can set $j=1$. Recall the two-dimensional Sobolev inequality, for $\varrho \in H^{1}\left(\mathbb{R}^{2}, \mathbb{C}\right)$ and for any $2<m<\infty$, there exists a positive constant $C_{m}$, such that $\|\varrho\|_{m} \leq C_{m}\|\nabla \varrho\|^{\frac{m-2}{m}}\|\varrho\|^{\frac{2}{m}}$ holds. Using Hölder and Sobolev for the $x_{1}$-integration, we get, for $p>1$

$$
\begin{aligned}
\left\|\mathbb{1}_{\overline{\mathcal{A}}_{1}^{(d)}} \Psi\right\|^{2} & =\left\langle\Psi, \mathbb{1}_{\overline{\mathcal{A}}_{1}^{(d)}} \Psi\right\rangle \\
& =\int d^{2} x_{2} \ldots d^{2} x_{N} \int d^{2} x_{1}\left|\Psi\left(x_{1}, \ldots, x_{N}\right)\right|^{2} \mathbb{1}_{\overline{\mathcal{A}}_{1}^{(d)}}\left(x_{1}, \ldots, x_{N}\right) \\
& \leq\left\|\mathbb{1}_{\overline{\mathcal{A}}_{1}^{(d)}}\right\|_{\frac{p}{p-1}, \infty} \int d^{2} x_{2} \ldots d^{2} x_{N}\left(\int d^{2} x_{1}\left|\Psi\left(x_{1}, \ldots, x_{N}\right)\right|^{2 p}\right)^{1 / p} \\
\leq & C_{p} N^{(1-2 d) \frac{p-1}{p}} \int d^{2} x_{2} \ldots d^{2} x_{N}\left(\int d^{2} x_{1}\left|\nabla_{1} \Psi\left(x_{1}, \ldots, x_{N}\right)\right|^{2}\right)^{\frac{p-1}{p}} \\
& \times\left(\int d^{2} \tilde{x}_{1}\left|\Psi\left(\tilde{x}_{1}, \ldots, x_{N}\right)\right|^{2}\right)^{\frac{1}{p}}
\end{aligned}
$$

where $C_{p}$ denotes a positive constant, depending on $p$.

Using Hölder for the $x_{2}, \ldots x_{N}$-integration with the conjugate pair $r=\frac{p}{p-1}$ and $s=p$, we then obtain

$$
\left\|\mathbb{1}_{\overline{\mathcal{A}}_{1}^{(d)}} \Psi\right\|^{2} \leq C_{p} N^{(1-2 d) \frac{p-1}{p}}\left\|\nabla_{1} \Psi\right\|^{2 \frac{p-1}{p}}\|\Psi\|^{\frac{2}{p}}
$$

(c) We use that $\overline{\mathcal{B}}_{j}^{(d)} \subset \bigcup_{k=1}^{N} \overline{\mathcal{A}}_{k}^{(d)}$. Hence one can find pairwise disjoint sets $\mathcal{C}_{k} \subset \overline{\mathcal{A}}_{k}^{(d)}$, $k=1, \ldots, N$ such that $\overline{\mathcal{B}}_{j}^{(d)} \subset \bigcup_{k=1}^{N} \mathcal{C}_{k}$. Since the sets $\mathcal{C}_{k}$ are pairwise disjoint, the $\mathbb{1}_{\mathcal{C}_{k}} \Psi$ are pairwise orthogonal and we get

$$
\left\|\mathbb{1}_{\overline{\mathcal{B}}_{j}^{(d)}} \Psi\right\|^{2}=\sum_{k=1}\left\|\mathbb{1}_{\mathcal{C}_{k}} \Psi\right\|^{2} \leq \sum_{k=1}^{N}\left\|\mathbb{1}_{\overline{\mathcal{A}}_{k}^{(d)}} \Psi\right\|^{2} .
$$

(d)

$$
\begin{aligned}
\left\|\left[\mathbb{1}_{\overline{\mathcal{A}}_{1}^{(d)}}, p_{2}\right]\right\|_{\text {op }} & \leq\left\|\left[\mathbb{1}_{a_{1,2}}, p_{2}\right]\right\|_{\text {op }} \leq\left\|\mathbb{1}_{a_{1,2}} p_{2}\right\|_{\text {op }}+\left\|p_{2} \mathbb{1}_{a_{1,2}}\right\|_{\text {op }} \\
& \leq 2\|\varphi\|_{\infty}\left|a_{1,2}\right|^{\frac{1}{2}} \leq C\|\varphi\|_{\infty} N^{-d}
\end{aligned}
$$


7.3. Proof of Lemma 6.6. The goal of this section is to prove Lemma 6.6. To this end, we bound each of the functionals $\gamma_{a}^{<}, \gamma_{b}^{<}$and $\gamma_{c}^{<}$separately and then collect the estimates. In view of the conditions required in Lemma 6.6, the following is assumed in the rest of this section:

Let $\beta>0, W_{\beta} \in \mathcal{W}_{\beta}, \varphi \in H^{3}\left(\mathbb{R}^{2}, \mathbb{C}\right)$ with $\|\varphi\|=1$ and $\Psi \in L_{s}^{2}\left(\mathbb{R}^{2 N}, \mathbb{C}\right) \cap$ $H^{2}\left(\mathbb{R}^{2 N}, \mathbb{C}\right)$ with $\|\Psi\|=1$ such that $\mathcal{E}_{W_{\beta}}(\Psi) \leq C$.

Control of $\gamma_{a}^{<}$

Lemma 7.5. For any function $B \in L^{\infty}\left(\mathbb{R}^{2}, \mathbb{R}\right)$, any $\varphi \in L^{2}\left(\mathbb{R}^{2}, \mathbb{C}\right)$ with $\|\varphi\|=1$ and any $\Psi \in L_{s}^{2}\left(\mathbb{R}^{2 N}, \mathbb{C}\right)$ with $\|\Psi\|=1$ we have

$$
\left|\left\langle\Psi, B\left(x_{1}\right) \Psi\right\rangle-\langle\varphi, B \varphi\rangle\right| \leq C\|B\|_{\infty}\left(\left\langle\Psi, \widehat{n}^{\varphi} \Psi\right\rangle+N^{-\frac{1}{2}}\right) .
$$

Proof. Using $1=p_{1}+q_{1}$,

$$
\begin{aligned}
& \left\langle\Psi \Psi, B\left(x_{1}\right) \Psi\right\rangle-\langle\varphi, B \varphi\rangle \\
& \quad=\left\langle\left\langle\Psi, p_{1} B\left(x_{1}\right) p_{1} \Psi\right\rangle+2 \Re\left\langle\left\langle\Psi, q_{1} B\left(x_{1}\right) p_{1} \Psi\right\rangle+\left\langle\left\langle\Psi, q_{1} B\left(x_{1}\right) q_{1} \Psi\right\rangle-\langle\varphi, B \varphi\rangle\right.\right.\right. \\
& \quad \leq\langle\varphi, B \varphi\rangle\left(\left\|p_{1} \Psi\right\|^{2}-1\right)+2 \Re\left\langle\left\langle\Psi, \widehat{n}^{-1 / 2} q_{1} B\left(x_{1}\right) p_{1} \widehat{n}_{1}^{1 / 2} \Psi\right\rangle+\left\langle\Psi, q_{1} B\left(x_{1}\right) q_{1} \Psi\right\rangle,\right.
\end{aligned}
$$

where we used Lemma 4.2 (c). Since $\left\|p_{1} \Psi\right\|^{2}-1=-\left\|q_{1} \Psi\right\|^{2}$ it follows that

$$
\begin{aligned}
\left|\left\langle\Psi \Psi, B\left(x_{1}\right) \Psi\right\rangle-\langle\varphi, B \varphi\rangle\right| & \leq C\|B\|_{\infty}\left(\left\langle\left\langle\Psi, \widehat{n}^{2} \Psi\right\rangle+\left\langle\left\langle\Psi, \widehat{n}_{1} \Psi\right\rangle+\langle\langle\Psi, \widehat{n} \Psi\rangle)\right.\right.\right. \\
& \leq C\|B\|_{\infty}\left(\langle\Psi, \widehat{n} \Psi\rangle+N^{-\frac{1}{2}}\right) .
\end{aligned}
$$

Using Lemma 7.5, $\|\widehat{n}-\widehat{m}\|_{o p} \leq C N^{-\xi}$ and setting $B=\dot{A}_{t}$, we get

$$
\left.\left|\gamma_{a}^{<}(\Psi, \varphi)\right| \leq C\left\|\dot{A}_{t}\right\|_{\infty}(\| \Psi, \widehat{n} \Psi\rangle+N^{-\frac{1}{2}}\right) \leq C\left\|\dot{A}_{t}\right\|_{\infty}\left(\alpha^{<}(\Psi, \varphi)+N^{-\xi}\right) .
$$

Control of $\gamma_{b}^{<}$To control $\gamma_{b}^{<}$we will first prove that $\left\|\nabla_{1} \Psi_{t}\right\|$ is uniformly bounded in $N$, if initially the energy per particle $\mathcal{E}_{U}\left(\Psi_{0}\right)$ is of order one.

Lemma 7.6. Let $\Psi_{0} \in L_{s}^{2}\left(\mathbb{R}^{2 N}, \mathbb{C}\right) \cap H^{2}\left(\mathbb{R}^{2 N}, \mathbb{C}\right)$ with $\left\|\Psi_{0}\right\|=1$. For any $U \in$ $L^{2}\left(\mathbb{R}^{2}, \mathbb{R}\right), U(x) \geq 0$, let $\Psi_{t}$ the unique solution to $i \partial_{t} \Psi_{t}=H_{U} \Psi_{t}$ with initial datum $\Psi_{0}$. Let $\mathcal{E}_{U}\left(\Psi_{0}\right) \leq C$. Then

$$
\left\|\nabla_{1} \Psi_{t}\right\| \leq \mathcal{K}\left(\varphi_{t}, A_{t}\right)
$$

Proof. Using $\frac{d}{d t} \mathcal{E}_{U}\left(\Psi_{t}\right) \leq\left\|\dot{A}_{t}\right\|_{\infty}$, we obtain $\mathcal{E}_{U}\left(\Psi_{t}\right) \leq \mathcal{K}\left(\varphi_{t}, A_{t}\right)$. This yields

$$
\left\|\nabla_{1} \Psi_{t}\right\|^{2} \leq \mathcal{K}\left(\varphi_{t}, A_{t}\right)-\frac{N-1}{2}\left\|\sqrt{U}\left(x_{1}-x_{2}\right) \Psi_{t}\right\|^{2}+\left\|A_{t}\right\|_{\infty} \leq \mathcal{K}\left(\varphi_{t}, A_{t}\right) .
$$


Next, we control $\widehat{m}^{a}$ and $\widehat{m}^{b}$ which were defined in Definition 6.4. The difference $m(k)-m(k+1)$ and $m(k)-m(k+2)$ is of leading order given by the derivative of the function $m(k)-k$ understood as real variable-with respect to $k$. The $k$-derivative of $m(k)$ equals

$$
m(k)^{\prime}= \begin{cases}1 /(2 \sqrt{k N}), & \text { for } k \geq N^{1-2 \xi} ; \\ 1 / 2\left(N^{-1+\xi}\right), & \text { else. }\end{cases}
$$

It is then easy to show that, for any $j \in \mathbb{Z}$, there exists a $C_{j}<\infty$ such that

$$
\begin{aligned}
\widehat{m}_{j}^{x} & \leq C_{j} N^{-1} \widehat{n}^{-1} \text { for } x \in\{a, b\} \\
\left\|\widehat{m}_{j}^{x}\right\|_{\text {op }} & \leq C_{j} N^{-1+\xi} \text { for } x \in\{a, b\} \\
\left\|\widehat{n}_{j}^{x}\right\|_{\text {op }} & \leq C_{j} N^{-1} \text { for } x \in\{a, b\} \\
\|\widehat{r}\|_{\text {op }} & \leq\left\|\widehat{m}^{a}\right\|_{\text {op }}+\left\|\widehat{m}^{b}\right\|_{\text {op }} \leq C N^{-1+\xi} .
\end{aligned}
$$

Now, we prove some general bounds, which will allow us to estimate the different terms of $\gamma_{b}^{<}$in (44). In order to facilitate the notation, let $\widehat{w} \in\left\{N \widehat{m}_{-1}^{a}, N \widehat{m}_{-2}^{b}\right\}$. Then $w(k)<C n(k)^{-1}$ and $\left\|\widehat{w}_{1}\right\|_{\text {op }} \leq C\|\widehat{w}\|_{\text {op }} \leq C N^{\xi}$ follows.

Lemma 7.7. Let $\beta>0$ and $W_{\beta} \in \mathcal{W}_{\beta}$. Let $\Psi \in L_{s}^{2}\left(\mathbb{R}^{2 N}, \mathbb{C}\right) \cap H^{2}\left(\mathbb{R}^{2 N}, \mathbb{C}\right)$ with $\|\Psi\|=1$ and let $\left\|\nabla_{1} \Psi\right\| \leq \mathcal{K}\left(\varphi, A_{t}\right)$. Let $w(k)<n(k)^{-1}$ and $\left\|\widehat{w}_{1}\right\|_{o p} \leq C\|\widehat{w}\|_{o p} \leq$ $C N^{\xi}$ for some $0<\xi<1 / 3$. Then,

(a)

$$
N\left|\left\langle\Psi, p_{1} p_{2} Z_{\beta}^{\varphi}\left(x_{1}, x_{2}\right) q_{1} p_{2} \widehat{w} \Psi\right\rangle\right| \leq \mathcal{K}\left(\varphi, A_{t}\right)\left(N^{-1}+N^{-2 \beta} \ln (N)\right) .
$$

(b)

$$
\begin{aligned}
& N\left|\left\langle\Psi, p_{1} p_{2} W_{\beta}\left(x_{1}-x_{2}\right) \widehat{w} q_{1} q_{2} \Psi\right\rangle\right| \leq \mathcal{K}\left(\varphi, A_{t}\right)(\langle\Psi, \widehat{n} \Psi\rangle \\
& \left.\quad+\inf _{\min \{\beta, 1 / 2\}>\beta_{1}>0} \inf _{\eta>0}\left(N^{\eta-2 \beta_{1}} \ln (N)^{2}+\|\widehat{w}\|_{o p} N^{-1+2 \beta_{1}}+\|\widehat{w}\|_{o p}^{2} N^{-\eta}\right)\right) .
\end{aligned}
$$

In addition, we have the slightly improved bound

$$
N\left|\left\langle\Psi, p_{1} p_{2} W_{\beta}\left(x_{1}-x_{2}\right) q_{1} q_{2} \widehat{w} \Psi\right\rangle\right| \leq \mathcal{K}\left(\varphi, A_{t}\right)\left(\langle\Psi, \widehat{n} \Psi\rangle+\|\widehat{w}\|_{o p} N^{-1+2 \beta}\right)
$$

(c)

$$
\begin{aligned}
& N \mid\left\langle\left\langle\Psi, p_{1} q_{2} Z_{\beta}^{\varphi}\left(x_{1}, x_{2}\right) \widehat{w} q_{1} q_{2} \Psi\right\rangle\right| \leq \mathcal{K}\left(\varphi, A_{t}\right)\left(\langle\Psi, \widehat{n} \Psi\rangle+N^{-1 / 6} \ln (N)\right. \\
& \left.\quad+\inf \left\{\left|\mathcal{E}_{V_{N}}(\Psi)-\mathcal{E}_{4 \pi}^{G P}(\varphi)\right|,\left|\mathcal{E}_{W_{\beta}}(\Psi)-\mathcal{E}_{b_{W_{\beta}}}^{G P}(\varphi)\right|+N^{-2 \beta} \ln (N)\right\}\right) .
\end{aligned}
$$

Proof. Since the left hand sides of all these statements are bounded, it follows that all these estimates hold uniformly in $N$ being in any finite subset of $\mathbb{N}$. Hence it suffices to prove the validity of (a), (b) and (c) for sufficiently large $N \in \mathbb{N}$. 
(a) In view of Lemma 4.4, we obtain

$$
\begin{aligned}
N\left|\left\langle\Psi, p_{1} p_{2} Z_{\beta}^{\varphi}\left(x_{1}, x_{2}\right) q_{1} p_{2} \widehat{w} \Psi\right\rangle\right| & \leq N\left\|p_{1} p_{2} Z_{\beta}^{\varphi}\left(x_{1}, x_{2}\right) q_{1} p_{2}\right\|_{\text {op }}\|\widehat{n} \widehat{w} \Psi\| \\
& \leq C N\left\|p_{1} p_{2} Z_{\beta}^{\varphi}\left(x_{1}, x_{2}\right) q_{1} p_{2}\right\|_{\mathrm{op}} .
\end{aligned}
$$

$\left\|p_{1} p_{2} Z_{\beta}^{\varphi}\left(x_{1}, x_{2}\right) q_{1} p_{2}\right\|_{\text {op }}$ can be estimated using $p_{1} q_{1}=0$ and (19):

$$
\begin{aligned}
N & \left\|p_{1} p_{2}\left(W_{\beta}\left(x_{1}-x_{2}\right)-\frac{N\left\|W_{\beta}\right\|_{1}}{N-1}\left|\varphi\left(x_{1}\right)\right|^{2}-\frac{N\left\|W_{\beta}\right\|_{1}}{N-1}\left|\varphi\left(x_{2}\right)\right|^{2}\right) q_{1} p_{2}\right\|_{\text {op }} \\
& \leq\left\|p_{1} p_{2}\left(N W_{\beta}\left(x_{1}-x_{2}\right)-N\left\|W_{\beta}\right\|_{1}\left|\varphi\left(x_{1}\right)\right|^{2}\right) p_{2}\right\|_{\text {op }}+C\|\varphi\|_{\infty}^{2} N^{-1} \\
& \leq\|\varphi\|_{\infty}\left\|N\left(W_{\beta} *|\varphi|^{2}\right)-\right\| N W_{\beta}\left\|_{1}|\varphi|^{2}\right\|+C\|\varphi\|_{\infty}^{2} N^{-1} .
\end{aligned}
$$

Let $h$ be given by

$$
h(x)=-\frac{1}{2 \pi} \int_{\mathbb{R}^{2}} d^{2} y \ln |x-y| N W_{\beta}(y)+\frac{1}{2 \pi}\left\|N W_{\beta}\right\|_{1} \ln |x|,
$$

which implies

$$
\Delta h(x)=N W_{\beta}(x)-\left\|N W_{\beta}\right\|_{1} \delta(x) .
$$

As above (see Lemma 7.2), we obtain $h(x)=0$ for $x \notin B_{R N^{-\beta}}(0)$, where $R N^{-\beta}$ is the radius of the support of $W_{\beta}$. Thus,

$$
\begin{aligned}
\|h\|_{1} \leq & \frac{1}{2 \pi} \int_{\mathbb{R}^{2}} d^{2} x \int_{\mathbb{R}^{2}} d^{2} y|\ln | x-y|| \mathbb{1}_{B_{R N^{-}}(0)}(x) N W_{\beta}(y) \\
& +\frac{1}{2 \pi} N\left\|W_{\beta}\right\|_{1} \int_{\mathbb{R}^{2}} d^{2} x|\ln | x|| \mathbb{1}_{B_{R N^{-}}(0)}(x) \leq C N^{-2 \beta} \ln (N) .
\end{aligned}
$$

Integration by parts and Young's inequality give that

$$
\begin{gathered}
\left\|N\left(W_{\beta} *|\varphi|^{2}\right)-\right\| N W_{\beta}\left\|_{1}|\varphi|^{2}\right\|=\left\|(\Delta h) *|\varphi|^{2}\right\| \\
\leq\|h\|_{1}\left\|\Delta|\varphi|^{2}\right\|_{2} \leq \mathcal{K}\left(\varphi, A_{t}\right) N^{-2 \beta} \ln (N) .
\end{gathered}
$$

Thus, we obtain the bound

$$
N\left|\left\langle\Psi, p_{1} p_{2} Z_{\beta}^{\varphi}\left(x_{1}, x_{2}\right) q_{1} p_{2} \widehat{w} \Psi\right\rangle\right| \leq \mathcal{K}\left(\varphi, A_{t}\right)\left(N^{-1}+N^{-2 \beta} \ln (N)\right),
$$

which then proves (a).

(b) We will first consider $\beta<1 / 2$.

Using Lemmas 4.2 (c) and 4.6 with $O_{1,2}=q_{2} W_{\beta}\left(x_{1}-x_{2}\right) p_{2}, \Omega=N^{-1 / 2}(\widehat{w})^{1 / 2} q_{1} \Psi$ 
and $\chi=N^{1 / 2} p_{1}\left(\widehat{w}_{2}\right)^{1 / 2} \Psi$ we get

$$
\begin{aligned}
\mid\langle\Psi \Psi & \left.p_{1} p_{2} W_{\beta}\left(x_{1}-x_{2}\right) q_{1} q_{2} \widehat{w} \Psi\right\rangle \mid \\
= & \left|\left\langle\Psi,(\widehat{w})^{1 / 2} q_{1} q_{2} W_{\beta}\left(x_{1}-x_{2}\right) p_{1} p_{2}\left(\widehat{w}_{2}\right)^{1 / 2} \Psi\right\rangle\right| \\
\leq & N^{-1}\left\|(\widehat{w})^{1 / 2} q_{1} \Psi\right\|^{2}+N \mid\left\langle\left\langle q_{2}\left(\widehat{w}_{2}\right)^{1 / 2} \Psi, p_{1} \sqrt{W_{\beta}}\left(x_{1}-x_{2}\right) p_{3} \sqrt{W_{\beta}}\left(x_{1}-x_{3}\right)\right.\right. \\
& \left.\sqrt{W_{\beta}}\left(x_{1}-x_{2}\right) p_{2} \sqrt{W_{\beta}}\left(x_{1}-x_{3}\right) p_{1} q_{3}\left(\widehat{w}_{2}\right)^{1 / 2} \Psi\right\rangle \mid \\
& +N(N-1)^{-1}\left\|q_{2} W_{\beta}\left(x_{1}-x_{2}\right) p_{2} p_{1}\left(\widehat{w}_{2}\right)^{1 / 2} \Psi\right\|^{2} \\
\leq & N^{-1}\left\|(\widehat{w})^{1 / 2} q_{1} \Psi\right\|^{2}+N\left\|\sqrt{W_{\beta}}\left(x_{1}-x_{2}\right) p_{1}\right\|_{\mathrm{op}}^{4}\left\|q_{2}\left(\widehat{w}_{2}\right)^{1 / 2} \Psi\right\|^{2} \\
& +2 N(N-1)^{-1}\left\|p_{1} q_{2}\left(\widehat{w}_{1}\right)^{1 / 2} W_{\beta}\left(x_{1}-x_{2}\right) p_{2} p_{1} \Psi\right\|^{2} \\
& +2 N(N-1)^{-1}\left\|q_{1} q_{2}(\widehat{w})^{1 / 2} W_{\beta}\left(x_{1}-x_{2}\right) p_{2} p_{1} \Psi\right\|^{2} .
\end{aligned}
$$

With Lemma 4.2 (e) we get the bound

$$
\begin{aligned}
\leq & N^{-1}\left\|(\widehat{w})^{1 / 2} \widehat{n} \Psi\right\|^{2}+N\|\varphi\|_{\infty}^{4}\left\|W_{\beta}\right\|_{1}^{2}\left\|\widehat{n}\left(\widehat{w}_{2}\right)^{1 / 2} \Psi\right\|^{2} \\
& +2 N(N-1)^{-1}\left\|W_{\beta}\right\|^{2}\|\varphi\|_{\infty}^{2}\left(\left\|\widehat{w}_{1}\right\|_{\text {op }}+\|\widehat{w}\|_{\text {op }}\right) .
\end{aligned}
$$

Note, that $\left\|W_{\beta}\right\|_{1} \leq C N^{-1},\left\|W_{\beta}\right\|^{2} \leq C N^{-2+2 \beta}$. Furthermore, using $\widehat{w}_{2} \leq(\widehat{n})^{-1}$, we have under the conditions on $\widehat{w}$

$$
\left\|\widehat{n}\left(\widehat{w}_{2}\right)^{1 / 2} \Psi\right\| \leq\left\|\widehat{n}(\widehat{n})^{-1 / 2} \Psi\right\|=\left\|(\widehat{n})^{1 / 2} \Psi\right\|=\sqrt{\langle\Psi, \widehat{n} \Psi\rangle} .
$$

In total, we obtain

$$
N\left|\left\langle\Psi \Psi, p_{1} p_{2} W_{\beta}\left(x_{1}-x_{2}\right) q_{1} q_{2} \widehat{w} \Psi\right\rangle\right| \leq \mathcal{K}\left(\varphi, A_{t}\right)\left(\langle\Psi \Psi, \widehat{n} \Psi\rangle+\|\widehat{w}\|_{\mathrm{op}} N^{-1+2 \beta}\right)
$$

and we get (b) for the case $\beta<1 / 2$.

(b) We prove part (b) for general $\beta>0$. We use $U_{\beta_{1}, \beta}$ from Definition 7.1 for some $0<\beta_{1}<\min \{\beta, 1 / 2\}$. We then obtain

$$
\begin{aligned}
& N\left\langle\Psi \Psi, p_{1} p_{2} W_{\beta}\left(x_{1}-x_{2}\right) \widehat{w} q_{1} q_{2} \Psi\right\rangle \\
& \quad=N\left\langle\left\langle\Psi, p_{1} p_{2} U_{\beta_{1}, \beta}\left(x_{1}-x_{2}\right) \widehat{w} q_{1} q_{2} \Psi\right\rangle\right. \\
& \quad+N\left\langle\Psi, p_{1} p_{2}\left(W_{\beta}\left(x_{1}-x_{2}\right)-U_{\beta_{1}, \beta}\left(x_{1}-x_{2}\right)\right) \widehat{w} q_{1} q_{2} \Psi\right\rangle .
\end{aligned}
$$

Term (82) has been controlled above. So we are left to control (83).

Let $\Delta h_{\beta_{1}, \beta}=W_{\beta}-U_{\beta_{1}, \beta}$. Integrating by parts and using that $\nabla_{1} h_{\beta_{1}, \beta}\left(x_{1}-x_{2}\right)=-\nabla_{2} h_{\beta_{1}, \beta}\left(x_{1}-x_{2}\right)$ gives

$$
\begin{aligned}
& N\left|\left\langle\Psi \Psi, p_{1} p_{2}\left(W_{\beta}\left(x_{1}-x_{2}\right)-U_{\beta_{1}, \beta}\left(x_{1}-x_{2}\right)\right) \widehat{w} q_{1} q_{2} \Psi\right\rangle\right| \\
& \leq N\left|\left\langle\nabla_{1} p_{1} \Psi, p_{2} \nabla_{2} h_{\beta_{1}, \beta}\left(x_{1}-x_{2}\right) \widehat{w} q_{1} q_{2} \Psi\right\rangle\right| \\
& \quad+N\left|\left\langle\Psi \Psi, p_{1} p_{2} \nabla_{2} h_{\beta_{1}, \beta}\left(x_{1}-x_{2}\right) \nabla_{1} \widehat{w} q_{1} q_{2} \Psi\right\rangle\right| .
\end{aligned}
$$


Let $t_{1} \in\left\{p_{1}, \nabla_{1} p_{1}\right\}$ and let $\Gamma \in\left\{\widehat{w} q_{1} \Psi, \nabla_{1} \widehat{w} q_{1} \Psi\right\}$.

For both (84) and (85), we use Lemma 4.6 with $O_{1,2}=N^{1+\eta / 2} q_{2} \nabla_{2} h_{\beta_{1}, \beta}\left(x_{1}-\right.$ $\left.x_{2}\right) p_{2}, \chi=t_{1} \Psi$ and $\Omega=N^{-\eta / 2} \Gamma$. This yields

$$
\begin{aligned}
(84)+(85) \leq & 2 \sup _{t_{1} \in\left\{p_{1}, \nabla_{1} p_{1}\right\}, \Gamma \in\left\{\widehat{w} q_{1} \Psi, \nabla_{1} \widehat{w} q_{1} \Psi\right\}}\left(N^{-\eta}\|\Gamma\|^{2}\right. \\
& +\frac{N^{2+\eta}}{N-1}\left\|q_{2} \nabla_{2} h_{\beta_{1}, \beta}\left(x_{1}-x_{2}\right) t_{1} p_{2} \Psi\right\|^{2} \\
& \left.+N^{2+\eta}\left|\left\langle\Psi \Psi, t_{1} p_{2} q_{3} \nabla_{2} h_{\beta_{1}, \beta}\left(x_{1}-x_{2}\right) \nabla_{3} h_{\beta_{1}, \beta}\left(x_{1}-x_{3}\right) t_{1} q_{2} p_{3} \Psi\right\rangle\right|\right) .
\end{aligned}
$$

The first term can be bounded using Corollary 4.5 by

$$
\begin{aligned}
N^{-\eta}\left\|\nabla_{1} \widehat{w} q_{1} \Psi\right\|^{2} & \leq 4 N^{-\eta}\|\widehat{w}\|_{\text {op }}^{2}\left\|\nabla_{1} q_{1} \Psi\right\|^{2} \\
N^{-\eta}\left\|\widehat{w} q_{1} \Psi\right\|^{2} & \leq C N^{-\eta} .
\end{aligned}
$$

Thus $(86) \leq \mathcal{K}\left(\varphi, A_{t}\right) N^{-\eta}\|\widehat{w}\|_{\text {op }}^{2}$ using that $\left\|\nabla_{1} q_{1} \Psi\right\| \leq \mathcal{K}\left(\varphi, A_{t}\right)$. By $\left\|t_{1} \Psi\right\|^{2} \leq$ $\mathcal{K}\left(\varphi, A_{t}\right)$, we obtain

$$
\begin{aligned}
(87) & \leq \mathcal{K}\left(\varphi, A_{t}\right) \frac{N^{2+\eta}}{N-1}\left\|\nabla_{2} h_{\beta_{1}, \beta}\left(x_{1}-x_{2}\right) p_{2}\right\|_{\mathrm{op}}^{2} \leq \mathcal{K}\left(\varphi, A_{t}\right) \frac{N^{2+\eta}}{N-1}\|\varphi\|_{\infty}^{2}\left\|\nabla h_{\beta_{1}, \beta}\right\|^{2} \\
& \leq \mathcal{K}\left(\varphi, A_{t}\right) N^{\eta-1} \ln (N),
\end{aligned}
$$

where we used Lemma 7.2 in the last step.

Next, we estimate

$$
\begin{aligned}
(88) \leq & N^{2+\eta}\left\|p_{2} \nabla_{2} h_{\beta_{1}, \beta}\left(x_{1}-x_{2}\right) t_{1} q_{2} \Psi\right\|^{2} \\
\leq & 2 N^{2+\eta}\left\|p_{2} h_{\beta_{1}, \beta}\left(x_{1}-x_{2}\right) t_{1} \nabla_{2} q 2 \Psi\right\|^{2} \\
& +2 N^{2+\eta} \|\left|\varphi\left(x_{2}\right)\right\rangle\left\langle\nabla \varphi\left(x_{2}\right)\right| h_{\beta_{1}, \beta}\left(x_{1}-x_{2}\right) t_{1} q_{2} \Psi \|^{2} \\
\leq & 2 N^{2+\eta}\left\|p_{2} h_{\beta_{1}, \beta}\left(x_{1}-x_{2}\right)\right\|_{\mathrm{op}}^{2}\left\|t_{1} \nabla_{2} q_{2} \Psi\right\|^{2} \\
& +2 N^{2+\eta} \|\left|\varphi\left(x_{2}\right)\right\rangle\left\langle\nabla \varphi\left(x_{2}\right)\right| h_{\beta_{1}, \beta}\left(x_{1}-x_{2}\right)\left\|_{\mathrm{op}}^{2}\right\| t_{1} q_{2} \Psi \|^{2} \\
\leq & \mathcal{K}\left(\varphi, A_{t}\right) N^{2+\eta}\left\|h_{\beta_{1}, \beta}\right\|^{2} \\
\leq & \mathcal{K}\left(\varphi, A_{t}\right) N^{\eta-2 \beta_{1}} \ln (N)^{2} .
\end{aligned}
$$

Thus, for all $\eta \in \mathbb{R}$

$$
\begin{aligned}
& \left.N \ll \Psi, p_{1} p_{2}\left(W_{\beta}\left(x_{1}-x_{2}\right)-U_{\beta_{1}, \beta}\left(x_{1}-x_{2}\right)\right) \widehat{w} q_{1} q_{2} \Psi\right\rangle \\
& \quad \leq \mathcal{K}\left(\varphi, A_{t}\right)\left(\|\widehat{w}\|_{\mathrm{op}}^{2} N^{-\eta}+N^{\eta-1} \ln (N)+N^{\eta-2 \beta_{1}} \ln (N)^{2}\right) .
\end{aligned}
$$

Combining the estimates and using $N^{\eta-1} \ln (N)<N^{\eta-2 \beta_{1}} \ln (N)^{2}$, we obtain

$$
\begin{aligned}
& N\left\langle\Psi, p_{1} p_{2} W_{\beta}\left(x_{1}-x_{2}\right) \widehat{w} q_{1} q_{2} \Psi\right\rangle \leq \mathcal{K}\left(\varphi, A_{t}\right)(\langle\Psi, \widehat{n} \Psi\rangle \\
& \left.\quad+\inf _{\min \{\beta, 1 / 2\}>\beta_{1}>0} \inf _{\eta>0}\left(N^{\eta-2 \beta_{1}} \ln (N)^{2}+\|\widehat{w}\|_{\mathrm{op}} N^{-1+2 \beta_{1}}+\|\widehat{w}\|_{\mathrm{op}}^{2} N^{-\eta}\right)\right) .
\end{aligned}
$$


(c) We note that $q_{1} p_{2}|\varphi|^{2}\left(x_{1}\right) q_{1} q_{2}=0$ and estimate

$$
\begin{aligned}
& N\left|\left\langle\Psi, q_{1} p_{2} \frac{N\left\|W_{\beta}\right\|_{1}}{N-1}|\varphi|^{2}\left(x_{2}\right) \widehat{w} q_{1} q_{2} \Psi\right\rangle\right| \leq C\|\varphi\|_{\infty}^{2}\|\widehat{w} \widehat{n}\|_{\text {op }}\left\|q_{1} \Psi\right\|^{2} \\
& \quad \leq \mathcal{K}\left(\varphi, A_{t}\right)\langle\Psi \Psi, \widehat{n} \Psi\rangle .
\end{aligned}
$$

Hence, it is left to estimate $N\left|\left\langle\Psi, q_{1} p_{2} W_{\beta}\left(x_{1}-x_{2}\right) \widehat{w} q_{1} q_{2} \Psi\right\rangle\right|$. Let $U_{0, \beta}$ be given as in Definition 7.1. Moreover, let $\mathcal{A}_{1}^{(d)}$ and $\overline{\mathcal{A}}_{1}^{(d)}$ be defined as in Definition 7.3 with $d \geq \max \{7,3+\beta\}$. We use Lemma 4.2 (c) and integrating by parts to get

$$
\begin{aligned}
N \mid\langle & \left.\Psi, q_{1} p_{2} W_{\beta}\left(x_{1}-x_{2}\right) \widehat{w} q_{1} q_{2} \Psi\right\rangle \mid \\
\leq & N\left|\left\langle\Psi, q_{1} p_{2} U_{0, \beta}\left(x_{1}-x_{2}\right) q_{1} q_{2} \widehat{w} \Psi\right\rangle\right| \\
& +N\left|\left\langle\Psi, q_{1} p_{2}\left(\Delta_{1} h_{0, \beta}\left(x_{1}-x_{2}\right)\right) q_{1} q_{2} \widehat{w} \Psi\right\rangle\right| \\
\leq & \left\|U_{0, \beta}\right\|_{\infty} N\left\|q_{1} \Psi\right\|\left\|\widehat{w} q_{1} q_{2} \Psi\right\| \\
& +N\left|\left\langle\nabla_{1} q_{1} p_{2} \Psi,\left(\nabla_{1} h_{0, \beta}\left(x_{1}-x_{2}\right)\right) \widehat{w} q_{1} q_{2} \Psi\right\rangle\right| \\
& +N\left|\left\langle\Psi \Psi, \widehat{w}_{1} q_{1} p_{2}\left(\nabla_{1} h_{0, \beta}\left(x_{1}-x_{2}\right)\right) \nabla_{1} q_{1} q_{2} \Psi\right\rangle\right| \\
\leq & N\left\|U_{0, \beta}\right\|_{\infty}\left\|q_{1} \Psi\right\|\left\|\widehat{w} q_{1} q_{2} \Psi\right\| \\
& +N\left|\left\langle\mathbb{1}_{\mathcal{A}_{1}^{(d)}} \nabla_{1} q_{1} \Psi, p_{2}\left(\nabla_{1} h_{0, \beta}\left(x_{1}-x_{2}\right)\right) \widehat{w} q_{1} q 2 \Psi\right\rangle\right| \\
& +N\left|\left\langle\nabla_{1} q_{1} \Psi, \mathbb{1}_{\overline{\mathcal{A}}_{1}^{(d)}} p_{2}\left(\nabla_{1} h_{0, \beta}\left(x_{1}-x_{2}\right)\right) q_{1} q_{2} \widehat{w} \Psi\right\rangle\right| \\
& +N\left|\left\langle\Psi \Psi, \widehat{w}_{1} q_{1} p_{2}\left(\nabla_{1} h_{0, \beta}\left(x_{1}-x_{2}\right)\right) q_{2} \mathbb{1}_{\overline{\mathcal{A}}_{1}^{(d)} \nabla_{1} q 1} \Psi\right\rangle\right| \\
& +N \mid\left\langle\Psi, \widehat{w}_{1} q_{1} p_{2}\left(\nabla_{1} h_{0, \beta}\left(x_{1}-x_{2}\right)\right) q_{2} \mathbb{1}_{\left.\mathcal{A}_{1}^{(d)} \nabla_{1} q_{1} \Psi\right\rangle \mid .}\right.
\end{aligned}
$$

In the following, we will estimate each term separately.

\section{Estimate of (89):}

Lemma 4.4 and Definition 7.1 yields the bound

$$
\text { (89) } \leq C\langle\Psi \Psi, \widehat{n} \Psi\rangle \text {. }
$$

\section{Estimate of (90):}

For (90) we use that $\nabla_{2} h_{0, \beta}\left(x_{1}-x_{2}\right)=-\nabla_{1} h_{0, \beta}\left(x_{1}-x_{2}\right)$, Cauchy Schwarz and $a b \leq a^{2}+b^{2}$ and get

$$
(90) \leq\left\|\mathbb{1}_{\mathcal{A}_{1}^{(d)}} \nabla_{1} q_{1} \Psi\right\|^{2}+N^{2}\left\|p_{2}\left(\nabla_{2} h_{0, \beta}\left(x_{1}-x_{2}\right)\right) \widehat{w} q_{1} q_{2} \Psi\right\|^{2} .
$$

$\left\|\mathbb{1}_{\mathcal{A}_{1}^{(d)}} \nabla_{1} q_{1} \Psi\right\|^{2}$ can be bounded using Lemma 7.9.

Integration by parts and Lemma 4.2 (c) as well as $(a+b)^{2} \leq 2 a^{2}+2 b^{2}$ gives for the 
second summand

$$
\begin{aligned}
N^{2} \| & p_{1}\left(\nabla_{1} h_{0, \beta}\left(x_{1}-x_{2}\right)\right) q_{1} q_{2} \widehat{w} \Psi \|^{2} \\
\leq & 2 N^{2}\left\|p_{1} h_{0, \beta}\left(x_{1}-x_{2}\right) \nabla_{1} q_{1} q_{2} \widehat{w} \Psi\right\|^{2} \\
& +2 N^{2} \|\left|\varphi\left(x_{1}\right)\right\rangle\left\langle\nabla_{1} \varphi\left(x_{1}\right)\right| h_{0, \beta}\left(x_{1}-x_{2}\right) q_{1} q_{2} \widehat{w} \Psi \|^{2} \\
\leq & C N^{2}\left\|p_{1} h_{0, \beta}\left(x_{1}-x_{2}\right) q_{2}\left(p_{1} \widehat{w}_{1}+q_{1} \widehat{w}\right) \mathbb{1}_{\mathcal{A}_{1}^{(d)}} \nabla_{1} q_{1} \Psi\right\|^{2} \\
& +C N^{2}\left\|p_{1} h_{0, \beta}\left(x_{1}-x_{2}\right) q_{2} p_{1} \widehat{w}_{1} \mathbb{1}_{\overline{\mathcal{A}}_{1}^{(d)}} \nabla_{1} q_{1} \Psi\right\|^{2} \\
& +C N^{2}\left\|p_{1} h_{0, \beta}\left(x_{1}-x_{2}\right) q_{2} q_{1} \widehat{w} \mathbb{1}_{\overline{\mathcal{A}}_{1}^{(d)}} \nabla_{1} q_{1} \Psi\right\|^{2} \\
& +2 N^{2} \|\left|\varphi\left(x_{1}\right)\right\rangle\left\langle\nabla_{1} \varphi\left(x_{1}\right)\right| h_{0, \beta}\left(x_{1}-x_{2}\right) q_{1} q_{2} \widehat{w} \Psi \|^{2} .
\end{aligned}
$$

For (95) we use Lemma 4.4, Lemma 4.2 (e) with Lemma 7.2 (c) and then Lemma 7.9.

$$
\begin{aligned}
(95) \leq & C N^{2}\left\|p_{1} h_{0, \beta}\left(x_{1}-x_{2}\right)\right\|_{\mathrm{op}}^{2}\left\|\mathbb{1}_{\mathcal{A}_{1}^{(d)}} \nabla_{1} q_{1} \Psi\right\|^{2} \\
\leq & \mathcal{K}\left(\varphi, A_{t}\right)\left(\left\langle\Psi, \widehat{n}^{\varphi} \Psi\right\rangle+N^{-1 / 6} \ln (N)\right. \\
& \left.+\inf \left\{\left|\mathcal{E}_{V_{N}}(\Psi)-\mathcal{E}_{4 \pi}^{G P}(\varphi)\right|,\left|\mathcal{E}_{W_{\beta}}(\Psi)-\mathcal{E}_{b_{W_{\beta}}}^{G P}(\varphi)\right|+N^{-2 \beta} \ln (N)\right\}\right) .
\end{aligned}
$$

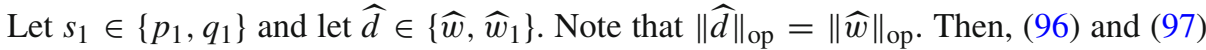
can be estimated with help of Lemma 7.4, part (b)

(96), (97) $\leq C N^{2}\left\|\nabla_{1} q_{1} \Psi\right\|\left\|\mathbb{1}_{\overline{\mathcal{A}}_{1}^{(d)}} \widehat{d}_{1} q_{2} h_{0, \beta}\left(x_{1}-x_{2}\right) p_{1} h_{0, \beta}\left(x_{1}-x_{2}\right) q_{2} s_{1} \widehat{d}_{\overline{\mathcal{A}}_{1}^{(d)}} \nabla_{1} q_{1} \Psi\right\|$ $\leq C_{p} N^{2+\frac{1-2 d}{2} \frac{p-1}{p}}\left\|\nabla_{1} q_{1} \Psi\right\|\left\|\nabla_{1} \widehat{d}_{1} q_{2} h_{0, \beta}\left(x_{1}-x_{2}\right) p_{1} h_{0, \beta}\left(x_{1}-x_{2}\right) q_{2} s_{1} \widehat{d} \mathbb{1}_{\overline{\mathcal{A}}_{1}^{(d)}} \nabla_{1} q_{1} \Psi\right\|^{\frac{p-1}{p}}$ $\times\left\|\widehat{d}_{s_{1}} q_{2} h_{0, \beta}\left(x_{1}-x_{2}\right) p_{1} h_{0, \beta}\left(x_{1}-x_{2}\right) q_{2} s_{1} \widehat{d}_{\overline{\mathcal{A}}_{1}^{(d)}} \nabla_{1} q_{1} \Psi\right\|^{\frac{1}{p}}$

$\leq C_{p} N^{2+\frac{1-2 d}{2} \frac{p-1}{p}}\left\|\nabla_{1} q_{1} \Psi\right\|\|\widehat{w}\|_{\text {op }}\left\|p_{1} h_{0, \beta}\left(x_{1}-x_{2}\right)\right\|_{\text {op }}\left\|\mathbb{1}_{\overline{\mathcal{A}}_{1}^{(d)}} \nabla_{1} q_{1} \Psi\right\|$ $\times\left\|\nabla_{1} \widehat{d} s_{1} q_{2} h_{0, \beta}\left(x_{1}-x_{2}\right) p_{1}\right\|_{\mathrm{op}}^{\frac{p-1}{p}}\left\|\widehat{d} s_{1} q_{2} h_{0, \beta}\left(x_{1}-x_{2}\right) p_{1}\right\|_{\mathrm{op}}^{\frac{1}{p}}$

$\leq C_{p} \mathcal{K}\left(\varphi, A_{t}\right) N^{1+\frac{1-2 d}{2} \frac{p-1}{p}}\|\widehat{w}\|_{\mathrm{op}}^{2}\left\|\nabla_{1} s_{1} h_{0, \beta}\left(x_{1}-x_{2}\right) p_{1}\right\|_{\mathrm{op}}^{\frac{p-1}{p}}\left\|h_{0, \beta}\left(x_{1}-x_{2}\right) p_{1}\right\|_{\mathrm{op}}^{\frac{1}{p}}$ $\leq C_{p} \mathcal{K}\left(\varphi, A_{t}\right) N^{1+\frac{1-2 d}{2} \frac{p-1}{p}}\|\widehat{w}\|_{\text {op }}^{2}\left(\|\nabla \varphi\|\left\|h_{0, \beta}\right\|+\left\|\nabla_{1} h_{0, \beta}\right\|\right)^{\frac{p-1}{p}}\left\|h_{0, \beta}\right\|^{\frac{1}{p}}$ $\leq C_{p} \mathcal{K}\left(\varphi, A_{t}\right)\|\widehat{w}\|_{\text {op }}^{2}(1+\ln (N))^{\frac{p-1}{2 p}} N^{\frac{1-2 d}{2} \frac{p-1}{p}}$.

Here, we used, for $s_{1} \in\left\{p_{1}, 1-p_{1}\right\}$,

$$
\begin{aligned}
\left\|\nabla_{1} s_{1} h_{0, \beta}\left(x_{1}-x_{2}\right) p_{1}\right\|_{\text {op }} & \leq\left\|\nabla_{1} p_{1} h_{0, \beta}\left(x_{1}-x_{2}\right) p_{1}\right\|_{\text {op }}+\left\|\nabla_{1} h_{0, \beta}\left(x_{1}-x_{2}\right) p_{1}\right\|_{\text {op }} \\
& \leq\|\varphi\|_{\infty}\left(\|\nabla \varphi\|\left\|h_{0, \beta}\right\|+\left\|\nabla h_{0, \beta}\right\|\right)
\end{aligned}
$$

and then applied Lemma 4.2 (e). With $\|\widehat{w}\|_{\text {op }} \leq N^{1 / 3}$, we obtain

$$
\begin{aligned}
(96)+(97) & \leq C_{p} \mathcal{K}\left(\varphi, A_{t}\right) \ln (N)^{\frac{p-1}{2 p}} N^{\frac{2}{3}} N^{\frac{1-2 d}{2} \frac{p-1}{p}} \\
& \leq C_{p} \mathcal{K}\left(\varphi, A_{t}\right) \ln (N)^{\frac{1}{2}} N^{2+\frac{d}{p}-d-\frac{1}{2 p}} .
\end{aligned}
$$


For $p=2$ and $d \geq \max \{7,3+\beta\}$, we obtain

$$
(96)+(97) \leq C_{2} \mathcal{K}\left(\varphi, A_{t}\right) N^{-1} \leq \mathcal{K}\left(\varphi, A_{t}\right) N^{-1} .
$$

Line (98) can be bounded by

$$
\begin{aligned}
(98) & \leq C N^{2}\left\|h_{0, \beta}\left(x_{1}-x_{2}\right) \nabla_{1} p_{1}\right\|_{\mathrm{op}}^{2}\left\|q_{1} q_{2} \widehat{w} \Psi\right\|^{2} \\
& \leq C N^{2}\left\|h_{0, \beta}\right\|^{2}\|\nabla \varphi\|_{\infty}^{2}\|\widehat{n} \widehat{w}\|_{\mathrm{op}}^{2}\left\|q_{1} \Psi\right\|^{2} \\
& \leq C\|\nabla \varphi\|_{\infty}^{2}\langle\Psi \widehat{n} \Psi\rangle .
\end{aligned}
$$

Estimate of (91) and (92):

For (91) and (92) we use Cauchy-Schwarz and then Sobolev inequality as in Lemma 7.4 implies that for any $p>1$, there exists a constant $C_{p}$ such that

$$
\begin{aligned}
(91)+(92) \leq & N\left\|\nabla_{1} q_{1} \Psi\right\|\left\|\mathbb{1}_{\overline{\mathcal{A}}_{1}^{(d)}} p_{2}\left(\nabla_{1} h_{0, \beta}\left(x_{1}-x_{2}\right)\right) q_{1} q_{2} \widehat{w} \Psi\right\| \\
& +N\left\|\nabla_{1} q_{1} \Psi\right\|\left\|\mathbb{1}_{\overline{\mathcal{A}}_{1}^{(d)}} q_{2}\left(\nabla_{1} h_{0, \beta}\left(x_{1}-x_{2}\right)\right) q_{1} p_{2} \widehat{w}_{1} \Psi\right\| \\
\leq & C_{p} N\left\|\nabla_{1} q_{1} \Psi\right\| N^{\frac{1-2 d}{2} \frac{p-1}{p}}\left\|\nabla_{1} p_{2}\left(\nabla_{1} h_{0, \beta}\left(x_{1}-x_{2}\right)\right) q_{1} q_{2} \widehat{w} \Psi\right\|^{\frac{p-1}{p}} \\
& \times\left\|p_{2}\left(\nabla_{1} h_{0, \beta}\left(x_{1}-x_{2}\right)\right) q_{1} q_{2} \widehat{w} \Psi\right\|^{1 / p} \\
& +C_{p} N\left\|\nabla_{1} q_{1} \Psi\right\| N^{\frac{1-2 d}{2} \frac{p-1}{p}}\left\|\nabla_{1} q_{2}\left(\nabla_{1} h_{0, \beta}\left(x_{1}-x_{2}\right)\right) q_{1} p_{2} \widehat{w}_{1} \Psi\right\|^{\frac{p-1}{p}} \\
& \times\left\|q_{2}\left(\nabla_{1} h_{0, \beta}\left(x_{1}-x_{2}\right)\right) q_{1} p_{2} \widehat{w}_{1} \Psi\right\|^{1 / p} .
\end{aligned}
$$

Using Lemma 4.2, Lemma 4.4, Corollary 4.5 and Lemma 7.2, we obtain

$$
\begin{aligned}
& \left\|\nabla_{1} p_{2}\left(\nabla_{1} h_{0, \beta}\left(x_{1}-x_{2}\right)\right) q_{1} q_{2} \widehat{w} \Psi\right\| \\
& \quad \leq\left\|p_{2}\left(\Delta_{1} h_{0, \beta}\left(x_{1}-x_{2}\right)\right) q_{1} q_{2} \widehat{w} \Psi\right\|+\left\|p_{2}\left(\nabla_{1} h_{0, \beta}\left(x_{1}-x_{2}\right)\right) \nabla_{1} q_{1} q_{2} \widehat{w} \Psi\right\| \\
& \quad \leq C\left(\left\|p_{2}\left(W_{\beta}-U_{0, \beta}\right)\left(x_{1}-x_{2}\right)\right\|_{\mathrm{op}}+\left\|p_{2}\left(\nabla_{1} h_{0, \beta}\left(x_{1}-x_{2}\right)\right)\right\|_{\mathrm{op}}\right) \\
& \quad \leq C\|\varphi\|_{\infty}\left(N^{-1+\beta}+N^{-1}(\ln (N))^{1 / 2}\right),
\end{aligned}
$$

and similarly

$$
\begin{aligned}
& \left\|\nabla_{1} q_{2}\left(\nabla_{1} h_{0, \beta}\left(x_{1}-x_{2}\right)\right) q_{1} p_{2} \widehat{w}_{1} \Psi\right\| \\
& \quad \leq\left\|q_{2}\left(\Delta_{1} h_{0, \beta}\left(x_{1}-x_{2}\right)\right) q_{1} p_{2} \widehat{w}_{1} \Psi\right\|+\left\|q_{2}\left(\nabla_{1} h_{0, \beta}\left(x_{1}-x_{2}\right)\right) \nabla_{1} q_{1} p_{2} \widehat{w}_{1} \Psi\right\| \\
& \quad \leq C\left(\left\|p_{2}\left(W_{\beta}-U_{0, \beta}\right)\left(x_{1}-x_{2}\right)\right\|_{\mathrm{op}}+\left\|\widehat{w}_{1}\right\|_{\mathrm{op}}\left\|p_{2}\left(\nabla_{1} h_{0, \beta}\left(x_{1}-x_{2}\right)\right)\right\|_{\mathrm{op}}\right) \\
& \quad \leq C\|\varphi\|_{\infty}\left(N^{-1+\beta}+\|\widehat{w}\|_{\mathrm{op}} N^{-1}(\ln (N))^{1 / 2}\right) .
\end{aligned}
$$

Moreover, we estimate

$$
\begin{aligned}
\left\|p_{2}\left(\nabla_{1} h_{0, \beta}\left(x_{1}-x_{2}\right)\right) q_{1} q_{2} \widehat{w} \Psi\right\| & \leq C\|\varphi\|_{\infty}\left\|\nabla_{1} h_{0, \beta}\right\|_{2} \leq C\|\varphi\|_{\infty} N^{-1}(\ln (N))^{1 / 2} \\
\left\|q_{2}\left(\nabla_{1} h_{0, \beta}\left(x_{1}-x_{2}\right)\right) q_{1} p_{2} \widehat{w}_{1} \Psi\right\| & \leq C\|\varphi\|_{\infty}\left\|\nabla_{1} h_{0, \beta}\right\|_{2} \leq C\|\varphi\|_{\infty} N^{-1}(\ln (N))^{1 / 2} .
\end{aligned}
$$

Thus if we choose $p=2$ and recall that $\xi<1 / 3$ and $d \geq \max \{7,3+\beta\}$, we obtain

$$
\begin{aligned}
(91)+(92) & \leq C_{2}\|\varphi\|_{\infty} N^{1+\frac{1-2 d}{4}}\left(N^{-1+\beta}+\|\widehat{w}\|_{\mathrm{op}} N^{-1}(\ln (N))^{1 / 2}\right)^{\frac{1}{2}}\left(N^{-1}(\ln (N))^{1 / 2}\right)^{1 / 2} \\
& \leq C_{2}\|\varphi\|_{\infty}\left(N^{\frac{1}{2}+\beta-d}(\ln (N))^{1 / 2}+N^{\frac{1}{3}+\frac{1}{2}-d} \ln (N)\right)^{\frac{1}{2}} \leq C\|\varphi\|_{\infty} N^{-1}
\end{aligned}
$$




\section{Estimate of (93):}

For (93) we use Lemma 4.6 with $\Omega=\mathbb{1}_{\mathcal{A}_{1}^{(d)}} \nabla_{1} q_{1} \Psi, O_{1,2}=N q_{2}\left(\nabla_{2} h_{0, \beta}\left(x_{1}-x_{2}\right)\right) p_{2}$ and $\chi=\widehat{w}_{1} q_{1} \Psi$.

$$
\begin{aligned}
(93) \leq & \left\|\mathbb{1}_{\mathcal{A}_{1}^{(d)}} \nabla_{1} q_{1} \Psi\right\|^{2} \\
& +2 N\left\|q_{2}\left(\nabla_{2} h_{0, \beta}\left(x_{1}-x_{2}\right)\right) \widehat{w}_{1} q_{1} p_{2} \Psi\right\|^{2} \\
& +N^{2}\left|\left\langle\Psi, q_{1} q_{3} \widehat{w}_{1}\left(\nabla_{2} h_{0, \beta}\left(x_{1}-x_{2}\right)\right) p_{2} p_{3}\left(\nabla_{3} h_{0, \beta}\left(x_{1}-x_{3}\right)\right) \widehat{w}_{1} q_{1} q_{2} \Psi\right\rangle\right| .
\end{aligned}
$$

Line (100) is bounded by

$$
\begin{aligned}
(100) & \leq C N\left\|\left(\nabla_{2} h_{0, \beta}\left(x_{1}-x_{2}\right)\right) p_{2}\right\|_{\mathrm{op}}^{2}\left\|\widehat{w}_{1} \widehat{n}\right\|_{\mathrm{op}}^{2} \\
& \leq C\|\varphi\|_{\infty}^{2} N\left\|\nabla_{2} h_{0, \beta}\left(x_{1}-x_{2}\right)\right\|^{2} \leq C\|\varphi\|_{\infty}^{2} N^{-1} \ln (N) .
\end{aligned}
$$

$(99)+(101)$ is bounded by

$$
\left\|\mathbb{1}_{\mathcal{A}_{1}^{(d)}} \nabla_{1} q_{1} \Psi\right\|^{2}+N^{2}\left\|p_{2}\left(\nabla_{2} h_{0, \beta}\left(x_{1}-x_{2}\right)\right) \widehat{w}_{1} q_{1} q_{2} \Psi\right\|^{2} .
$$

Both terms can be controlled analogously to (94).

\section{Complete estimate:}

In total, we obtain

$$
\begin{aligned}
& N\left|\left\langle\Psi, p_{1} q_{2} Z_{\beta}^{\varphi}\left(x_{1}, x_{2}\right) \widehat{w} q_{1} q_{2} \Psi\right\rangle\right| \leq \mathcal{K}\left(\varphi, A_{t}\right)\left(\langle\Psi, \widehat{n} \Psi\rangle+N^{-1 / 6} \ln (N)\right. \\
& \left.\quad+\inf \left\{\left|\mathcal{E}_{V_{N}}(\Psi)-\mathcal{E}_{4 \pi}^{G P}(\varphi)\right|,\left|\mathcal{E}_{W_{\beta}}(\Psi)-\mathcal{E}_{b_{W_{\beta}}}^{G P}(\varphi)\right|+N^{-2 \beta} \ln (N)\right\}\right) .
\end{aligned}
$$

To estimate $\gamma_{b}^{<}$we recall that $\widehat{w} \in\left\{N \widehat{m}_{-1}^{a}, N \widehat{m}_{-2}^{b}\right\}$ with $w(k)<n(k)^{-1}$ and $\left\|\widehat{w}_{1}\right\|_{\text {op }} \leq$ $C\|\widehat{w}\|_{\text {op }} \leq C N^{\xi}$. Lemma 7.7, $\|\widehat{n}-\widehat{m}\|_{o p} \leq C N^{-\xi}$ and $\xi<1 / 3$ imply

$$
\begin{aligned}
& \gamma_{b}^{<}(\Psi, \varphi) \leq \mathcal{K}\left(\varphi, A_{t}\right)\left(\alpha^{<}(\Psi, \varphi)+N^{-1 / 3}\right. \\
& \left.\quad+\inf _{\min \{\beta, 1 / 2\}>\beta_{1}>0} \inf _{\eta>0}\left(N^{\eta-2 \beta_{1}} \ln (N)^{2}+N^{-1+\xi+2 \beta_{1}}+N^{2 \xi-\eta}\right)\right) .
\end{aligned}
$$

In addition, we have the improved bound

$$
\gamma_{b}^{<}(\Psi, \varphi) \leq \mathcal{K}\left(\varphi, A_{t}\right)\left(\alpha^{<}(\Psi, \varphi)+N^{-\xi}+N^{-1+\xi+2 \beta}+\left(N^{-1 / 6}+N^{-2 \beta}\right) \ln (N)\right)
$$

for al $\beta<1 / 2$.

Control of $\gamma_{c}^{<}$With Definition 2.1 and (76) we estimate

$$
\begin{aligned}
\left|\gamma_{c}^{<}(\Psi, \varphi)\right| & \leq N\left|N\left\|W_{\beta}\right\|_{1}-b_{W_{\beta}}\right|\left|\left\langle\Psi,\left(q_{1}\left|\varphi\left(x_{1}\right)\right|^{2} \widehat{m}^{a} p_{1}-p_{1} \widehat{m}^{a}\left|\varphi\left(x_{1}\right)\right|^{2} q_{1}\right) \Psi\right\rangle\right| \\
& \leq N\left|N\left\|W_{\beta}\right\|_{1}-b_{W_{\beta}}\right|\|\varphi\|_{\infty}^{2}\left\|\widehat{m}^{a}\right\|_{\mathrm{op}}\left\|q_{1} \Psi\right\| \\
& \leq \mathcal{K}\left(\varphi, A_{t}\right) N^{-1+\xi} \ln (N) .
\end{aligned}
$$

Collecting all the estimates for $\gamma_{a}^{<}, \gamma_{b}^{<}$and $\gamma_{c}^{<}$then proves Lemma 6.6. 
Proof of Lemma 6.6. Let the assumptions of Lemma 6.6 be satisfied. By the previous we have

$$
\begin{aligned}
& \sum_{k \in\{a, b, c\}}\left|\gamma_{k}^{<}\left(\Psi_{t}, \varphi_{t}\right)\right| \leq \mathcal{K}\left(\varphi_{t}, A_{t}\right)\left(\alpha^{<}\left(\Psi_{t}, \varphi_{t}\right)+N^{-\xi}+\left(N^{-1 / 6}+N^{-2 \beta}\right) \ln (N)\right. \\
& \left.\quad+\inf _{\min \{\beta, 1 / 2\}>\beta_{1}>0} \inf _{\eta>0}\left(N^{\eta-2 \beta_{1}} \ln (N)^{2}+N^{-1+\xi+2 \beta_{1}}+N^{2 \xi-\eta}\right)\right)
\end{aligned}
$$

and the slightly stronger estimate

$$
\begin{aligned}
& \sum_{k \in\{a, b, c\}}\left|\gamma_{k}^{<}\left(\Psi_{t}, \varphi_{t}\right)\right| \leq \mathcal{K}\left(\varphi_{t}, A_{t}\right)\left(\alpha^{<}\left(\Psi_{t}, \varphi_{t}\right)+N^{-\xi}+N^{-1+\xi+2 \beta}\right. \\
& \left.\quad+\left(N^{-1 / 6}+N^{-2 \beta}\right) \ln (N)\right)
\end{aligned}
$$

if $\beta<1 / 2$. Inequality (49) follows for $1 / 3 \leq \beta$ from the first bound (with $\beta_{1}=3 / 10$ and $\eta=3 / 10$ ) and for $1 / 12 \leq \beta<1 / 3$ from the second relation. Moreover, if we choose $\beta<1 / 12$ and $\xi=1 / 6$ we obtain (48).

7.4. Proof of Lemma 6.13. Next, we prove Lemma 6.13. We will proceed in a similar way as in the previous section and consecutively estimate the functionals $\gamma_{i}$ with $i \in$ $\{a, b, c, d, e, f\}$. In the rest of this section we assume that $V_{N} \in \mathcal{V}_{N}, \varphi \in H^{3}\left(\mathbb{R}^{2}, \mathbb{C}\right)$ with $\|\varphi\|=1$ and that $\Psi \in L_{s}^{2}\left(\mathbb{R}^{2 N}, \mathbb{C}\right) \cap H^{2}\left(\mathbb{R}^{2 N}, \mathbb{C}\right)$ with $\|\Psi\|=1$ such that $\mathcal{E}_{V_{N}}(\Psi) \leq C$.

For the most involved scaling which is induced by $V_{N}$, we need to control $\| p_{1} V_{N}\left(x_{1}-\right.$ $\left.x_{2}\right) \Psi \|$.

Lemma 7.8. Let $V_{N} \in \mathcal{V}_{N}, \Psi \in L_{S}^{2}\left(\mathbb{R}^{2 N}, \mathbb{C}\right) \cap H^{1}\left(\mathbb{R}^{2 N}, \mathbb{C}\right), \varphi \in H^{3}\left(\mathbb{R}^{2}, \mathbb{C}\right)$ with $\|\varphi\|=1$ and $\mathcal{E}_{V_{N}}(\Psi) \leq C$. Then

$$
\left\|p_{1} V_{N}\left(x_{1}-x_{2}\right) \Psi\right\| \leq \mathcal{K}\left(\varphi, A_{t}\right) N^{-\frac{1}{2}} .
$$

Proof. We estimate

$$
\begin{aligned}
\left\|p_{1} V_{N}\left(x_{1}-x_{2}\right) \Psi\right\| & =\left\|p_{1} \mathbb{1}_{\operatorname{supp}\left(V_{N}\right)}\left(x_{1}-x_{2}\right) V_{N}\left(x_{1}-x_{2}\right) \Psi\right\| \\
& \leq\left\|p_{1} \mathbb{1}_{\operatorname{supp}\left(V_{N}\right)}\left(x_{1}-x_{2}\right)\right\|_{\text {op }}\left\|V_{N}\left(x_{1}-x_{2}\right) \Psi\right\| .
\end{aligned}
$$

With Lemma 4.2 (e) we get

$$
\left\|p_{1} \mathbb{1}_{\operatorname{supp}\left(V_{N}\right)}\left(x_{1}-x_{2}\right)\right\|_{\mathrm{op}}^{2} \leq\|\varphi\|_{\infty}^{2}\left\|\mathbb{1}_{\operatorname{supp}\left(V_{N}\right)}\right\|_{1} \leq C\|\varphi\|_{\infty}^{2} e^{-2 N} .
$$

Using

$$
C \geq \mathcal{E}_{V_{N}}(\Psi)=\|\nabla \Psi\|^{2}+\frac{(N-1)}{2}\left\|\sqrt{V_{N}}\left(x_{1}-x_{2}\right) \Psi\right\|^{2}+\left\langle\Psi \Psi, A_{t}\left(x_{1}\right) \Psi\right\rangle
$$

as well as

$$
\begin{aligned}
\left\|V_{N}\left(x_{1}-x_{2}\right) \Psi\right\|^{2} & =\left\|\sqrt{V_{N}}\left(x_{1}-x_{2}\right) \sqrt{V_{N}}\left(x_{1}-x_{2}\right) \Psi\right\|^{2} \\
& \leq\left\|\sqrt{V_{N}}\right\|_{\infty}^{2}\left\|\sqrt{V_{N}}\left(x_{1}-x_{2}\right) \Psi\right\|^{2} \\
& \leq C e^{2 N} \frac{\mathcal{E}_{V_{N}}(\Psi)+\left\|A_{t}\right\|_{\infty}}{N} \leq C\left(1+\left\|A_{t}\right\|_{\infty}\right) \frac{e^{2 N}}{N},
\end{aligned}
$$


we obtain

$$
\left\|p_{1} V_{N}\left(x_{1}-x_{2}\right) \Psi\right\| \leq \mathcal{K}\left(\varphi, A_{t}\right) N^{-\frac{1}{2}}
$$

\section{Control of $\gamma_{a}$}

In total analogy to (73) we get

$$
\left|\gamma_{a}(\Psi, \varphi)\right| \leq C\left\|\dot{A}_{t}\right\|_{\infty}\left(\langle\Psi, \widehat{n} \Psi\rangle+N^{-\frac{1}{2}}\right) \leq C\left\|\dot{A}_{t}\right\|_{\infty}\left(\langle\Psi, \widehat{m} \Psi\rangle+N^{-\xi}\right) .
$$

With Definition 6.8 and (58) we have

$$
\left|\gamma_{a}(\Psi, \varphi)\right| \leq \mathcal{K}\left(\varphi, A_{t}\right)\left(\alpha(\Psi, \varphi)+N^{-\xi}+N^{-\mu+\xi} \ln (N)\right) .
$$

Control of $\gamma_{b}$

Recall that

$$
\begin{aligned}
\gamma_{b}(\Psi, \varphi)= & -N(N-1) \mathfrak{s}\left(\left\langle\Psi, \widetilde{Z}_{\mu}^{\varphi}\left(x_{1}, x_{2}\right) \widehat{r} \Psi\right\rangle\right) \\
& -N(N-1) \mathfrak{\Im}\left(\left\langle\Psi, g_{\mu}\left(x_{1}-x_{2}\right) \widehat{r} \mathcal{Z}^{\varphi}\left(x_{1}, x_{2}\right) \Psi\right\rangle\right) .
\end{aligned}
$$

Estimate (102) yields to the bound $\left\|p_{1} \mathcal{Z}^{\varphi}\left(x_{1}, x_{2}\right) \Psi\right\| \leq \mathcal{K}\left(\varphi, A_{t}\right) N^{-1 / 2}$. Thus, if we use Lemma 5.5 and $\left\|\widehat{m}^{a}\right\|_{\text {op }}+\left\|\widehat{m}^{b}\right\|_{\text {op }} \leq C N^{-1+\xi}$ [see (76)] the second line is controlled by

$$
\begin{aligned}
& N^{2}\left(\left\|\widehat{m}^{a}\right\|_{\text {op }}+\left\|\widehat{m}^{b}\right\|_{\text {op }}\right)\left\|g_{\mu}\left(x_{1}-x_{2}\right) p_{1}\right\|_{\text {op }}\left\|p_{1} \mathcal{Z}^{\varphi}\left(x_{1}, x_{2}\right) \Psi\right\| \\
& \leq \mathcal{K}\left(\varphi, A_{t}\right) N^{1 / 2+\xi}\left\|g_{\mu}\right\| \leq \mathcal{K}\left(\varphi, A_{t}\right) N^{\xi-1 / 2-\mu} \ln (N) .
\end{aligned}
$$

The first line of $\gamma_{b}$ can be bounded with (62) and $f_{\mu}=1-g_{\mu}$ by

$$
\begin{aligned}
N(N-1)\left|\Im\left(\left\langle\Psi, \widetilde{Z}_{\mu}^{\varphi}\left(x_{1}, x_{2}\right) \widehat{r} \Psi\right\rangle\right)\right| \\
\leq N^{2} \mid \Im\left(\left\langle\Psi,\left(M_{\mu}\left(x_{1}-x_{2}\right) f_{\mu}\left(x_{1}-x_{2}\right)\right.\right.\right. \\
\left.\left.\left.\quad-\frac{N}{N-1}\left\|M_{\mu} f_{\mu}\right\|_{1}\left(\left|\varphi\left(x_{1}\right)\right|^{2}+\left|\varphi\left(x_{2}\right)\right|^{2}\right)\right) \widehat{r} \Psi\right\rangle\right) \mid \\
\quad+\frac{N^{2}}{N-1} \mid\left\langle\left\langle\Psi,\left(\left\|N M_{\mu} f_{\mu}\right\|_{1}-4 \pi\right)\left(\left|\varphi\left(x_{1}\right)\right|^{2}+\left|\varphi\left(x_{2}\right)\right|^{2}\right) \widehat{r} \Psi\right\rangle\right| \\
\quad+\frac{N^{2}}{N-1} \mid\left\langle\left\langle\Psi, 4 \pi\left(\left|\varphi\left(x_{1}\right)\right|^{2}+\left|\varphi\left(x_{2}\right)\right|^{2}\right) g_{\mu}\left(x_{1}-x_{2}\right) \widehat{r} \Psi\right\rangle\right| .
\end{aligned}
$$

Since $M_{\mu} f_{\mu} \in \mathcal{W}_{\mu},(103)$ is of the same form as $\gamma_{b}^{<}(\Psi, \varphi)$. By means of Lemma 7.7, $\|\widehat{n}-\widehat{m}\|_{o p} \leq C N^{-\xi}$ and (58), we obtain

$$
\begin{aligned}
|(103)| \leq & \mathcal{K}\left(\varphi, A_{t}\right)\left(\alpha(\Psi, \varphi)+N^{-\xi}+\left(N^{-1 / 6}+N^{-\mu+\xi}\right) \ln (N)\right. \\
& \left.+{\operatorname{minf}\{\beta, 1 / 2\}>\beta_{1}>0}_{\inf _{\eta>0}}\left(N^{\eta-2 \beta_{1}} \ln (N)^{2}+N^{-1+2 \beta_{1}+\xi}+N^{2 \xi-\eta}\right)\right) .
\end{aligned}
$$

Using Lemma $5.5(\mathrm{~h})$, the second term is controlled by

$$
(104) \leq C\|\varphi\|_{\infty}^{2} N\left|N\left\|M_{\mu} f_{\mu}\right\|_{1}-4 \pi\right|\|\hat{r}\|_{\text {op }} \leq C\|\varphi\|_{\infty}^{2} N^{-1+\xi} \ln (N) .
$$


The last term is controlled by

$$
(105) \leq C N\|\varphi\|_{\infty}^{2}\left\|g_{\mu}\left(x_{1}-x_{2}\right) p_{1}\right\|_{\text {op }}\left(\left\|\widehat{m}^{a}\right\|_{\text {op }}+\left\|\widehat{m}^{b}\right\|_{\text {op }}\right) \leq C\|\varphi\|_{\infty}^{3} N^{-1-\mu+\xi} \ln (N)
$$

which implies the bound

$$
\begin{aligned}
\left|\gamma_{b}(\Psi, \varphi)\right| \leq & \mathcal{K}\left(\varphi, A_{t}\right)\left(\alpha(\Psi, \varphi)+N^{-\xi}+\left(N^{-1+\xi}+N^{-1 / 6}+N^{-\mu+\xi}\right) \ln (N)\right. \\
& \left.+{\min \{\beta, 1 / 2\}>\beta_{1}>0}_{\inf _{\eta>0}}\left(N^{\eta-2 \beta_{1}} \ln (N)^{2}+N^{-1+2 \beta_{1}+\xi}+N^{2 \xi-\eta}\right)\right) .
\end{aligned}
$$

\section{Control of $\gamma_{c}$}

Recall that

$$
\gamma_{c}(\Psi, \varphi)=-4 N(N-1)\left\langle\Psi \Psi,\left(\nabla_{1} g_{\mu}\left(x_{1}-x_{2}\right)\right) \nabla_{1} \widehat{r} \Psi\right\rangle .
$$

Using $\widehat{r}=\left(p_{2}+q_{2}\right) \widehat{r}=p_{2} \widehat{r}+p_{1} q_{2} \widehat{m}^{a}$ and $\nabla_{1} g_{\mu}\left(x_{1}-x_{2}\right)=-\nabla_{2} g_{\mu}\left(x_{1}-x_{2}\right)$, integration by parts yields to

$$
\begin{aligned}
\left|\gamma_{c}(\Psi, \varphi)\right| \leq & 4 N^{2}\left|\left\langle\Psi \Psi, g_{\mu}\left(x_{1}-x_{2}\right) \nabla_{1} \nabla_{2}\left(p_{2} \widehat{r}+p_{1} q_{2} \widehat{m}^{a}\right) \Psi\right\rangle\right| \\
& +4 N^{2} \mid\left\langle\left\langle\nabla_{2} \Psi, g_{\mu}\left(x_{1}-x_{2}\right) \nabla_{1} p_{2} \widehat{r} \Psi\right\rangle\right| \\
& +4 N^{2} \mid\left\langle\left\langle\nabla_{2} \Psi, g_{\mu}\left(x_{1}-x_{2}\right) \nabla_{1} p_{1} q_{2} \widehat{m}^{a} \Psi\right\rangle\right| .
\end{aligned}
$$

We begin with

$$
\begin{aligned}
(106) & \leq C N^{2}\left\|g_{\mu}\right\|\|\nabla \varphi\|_{\infty}\left(\left\|\nabla_{1} \widehat{r} \Psi\right\|+\left\|\nabla_{2} q_{2} \widehat{m}^{a} \Psi\right\|\right) \\
& \leq C N^{1-\mu} \ln (N)\|\nabla \varphi\|_{\infty}\left(\left\|\nabla_{1} \widehat{r} \Psi\right\|+\left\|\nabla_{2} q_{2} \widehat{m}^{a} \Psi\right\|\right) .
\end{aligned}
$$

Let $s_{1}, t_{1} \in\left\{p_{1}, q_{1}\right\}$. Inserting the identity $1=p_{1}+q_{1}$, we obtain for $a \in\{-1,0,1\}$,

$$
\left\|\nabla_{1} \widehat{r} \Psi\right\| \leq C \sup _{s_{1}, t_{1}, a}\left\|\widehat{r}_{a} s_{1} \nabla_{1} t_{1} \Psi\right\| \leq C \sup _{t_{1}, a}\left\|\widehat{r}_{a}\right\|_{\mathrm{op}}\left\|\nabla_{1} t_{1} \Psi\right\| \leq C N^{-1+\xi} .
$$

In analogy $\left\|\nabla_{2} q_{2} \widehat{m}^{a} \Psi\right\| \leq C\left\|\widehat{m}^{a}\right\|_{\text {op }} \leq C N^{-1+\xi}$. This yields the bound

$$
(106) \leq \mathcal{K}\left(\varphi, A_{t}\right) N^{-\mu+\xi} \ln (N) .
$$

Furthermore, (107) is bounded by

$$
(107) \leq 4 N^{2}\left\|\nabla_{2} \Psi\right\|\left\|g_{\mu}\right\|\|\varphi\|_{\infty}\left\|\nabla_{1} \widehat{r} \Psi\right\| \leq C\|\varphi\|_{\infty} N^{\xi-\mu} \ln (N) .
$$

Similarly, we obtain

$$
(108) \leq 4 N^{2}\left\|\nabla_{2} \Psi\right\|\left\|g_{\mu}\right\|\|\nabla \varphi\|_{\infty}\left\|q_{2} \widehat{m}^{a} \Psi\right\| \leq C\|\nabla \varphi\|_{\infty} N^{\xi-\mu} \ln (N) .
$$

It follows that $\left|\gamma_{c}(\Psi, \varphi)\right| \leq \mathcal{K}\left(\varphi, A_{t}\right) N^{\xi-\mu} \ln (N)$.

\section{Control of $\gamma_{d}$}

To control $\gamma_{d}$ and $\gamma_{e}$ we will use the notation

$$
\begin{array}{ll}
m^{c}(k)=m^{a}(k)-m^{a}(k+1) & m^{d}(k)=m^{a}(k)-m^{a}(k+2) \\
m^{e}(k)=m^{b}(k)-m^{b}(k+1) & m^{f}(k)=m^{b}(k)-m^{b}(k+2) .
\end{array}
$$


Since the second $k$-derivative of $m$ is given by (see (74) for the first derivative)

$$
m(k)^{\prime \prime}= \begin{cases}-1 /\left(4 \sqrt{k^{3} N}\right), & \text { for } k \geq N^{1-2 \xi} ; \\ 0, & \text { else. }\end{cases}
$$

it is easy to verify that

$$
\left\|\widehat{m}_{j}^{x}\right\|_{\mathrm{op}} \leq C N^{-2+3 \xi} \text { for } x \in\{c, d, e, f\} .
$$

Recall that

$$
\begin{aligned}
\gamma_{d}(\Psi, \varphi)= & 2 N(N-1)(N-2) \mathfrak{\Im}\left(\left\langle\Psi, g_{\mu}\left(x_{1}-x_{2}\right)\left[V_{N}\left(x_{1}-x_{3}\right), \widehat{r}\right] \Psi\right\rangle\right) \\
& -N(N-1)(N-2) \Im\left(\left\langle\Psi, g_{\mu}\left(x_{1}-x_{2}\right)\left[4 \pi|\varphi|^{2}\left(x_{3}\right), \widehat{r}\right] \Psi\right\rangle\right) .
\end{aligned}
$$

Since $p_{j}+q_{j}=1$, we can rewrite $\widehat{r}$ as

$$
\widehat{r}=\widehat{m}^{b} p_{1} p_{2}+\widehat{m}^{a}\left(p_{1} q_{2}+q_{1} p_{2}\right)=\left(\widehat{m}^{b}-2 \widehat{m}^{a}\right) p_{1} p_{2}+\widehat{m}^{a}\left(p_{1}+p_{2}\right) .
$$

Thus,

$$
\begin{aligned}
\left|\gamma_{d}(\Psi, \varphi)\right| \leq & C N^{3} \mid\left\langle\Psi, g_{\mu}\left(x_{1}-x_{2}\right)\left[V_{N}\left(x_{1}-x_{3}\right),\left(\widehat{m}^{b}-2 \widehat{m}^{a}\right) p_{1} p_{2}\right.\right. \\
& \left.\left.+\widehat{m}^{a}\left(p_{1}+p_{2}\right)\right] \Psi\right\rangle \mid \\
& +C N^{3}\left|\left\langle\Psi, g_{\mu}\left(x_{1}-x_{2}\right)\left[4 \pi|\varphi|^{2}\left(x_{3}\right), \widehat{r}\right] \Psi\right\rangle\right| \\
\leq & C N^{3}\left|\left\langle\Psi, g_{\mu}\left(x_{1}-x_{2}\right) p_{2}\left[V_{N}\left(x_{1}-x_{3}\right), \widehat{m}^{a}\right] \Psi\right\rangle\right| \\
& +C N^{3}\left|\left\langle\Psi, g_{\mu}\left(x_{1}-x_{2}\right) V_{N}\left(x_{1}-x_{3}\right)\left(\widehat{m}^{b}-2 \widehat{m}^{a}\right) p_{1} p_{2} \Psi\right\rangle\right| \\
& +C N^{3}\left|\left\langle\Psi, g_{\mu}\left(x_{1}-x_{2}\right)\left(\widehat{m}^{b}-2 \widehat{m}^{a}\right) p_{1} p_{2} V_{N}\left(x_{1}-x_{3}\right) \Psi\right\rangle\right| \\
& +C N^{3}\left|\left\langle\Psi \Psi, g_{\mu}\left(x_{1}-x_{2}\right) \widehat{m}^{a} p_{1} V_{N}\left(x_{1}-x_{3}\right) \Psi\right\rangle\right| \\
& +C N^{3}\left|\left\langle\Psi \Psi, g_{\mu}\left(x_{1}-x_{2}\right) V_{N}\left(x_{1}-x_{3}\right) \widehat{m}^{a} p_{1} \Psi\right\rangle\right| \\
& +C N^{3}\left|\left\langle\Psi, g_{\mu}\left(x_{1}-x_{2}\right)\left[4 \pi|\varphi|^{2}\left(x_{3}\right), \widehat{r}\right] \Psi\right\rangle\right| .
\end{aligned}
$$

Using Lemma 4.2 (d), we obtain the following estimate:

$$
\begin{aligned}
(112)= & C N^{3}\left|\left\langle\Psi, g_{\mu}\left(x_{1}-x_{2}\right) p_{2}\left[V_{N}\left(x_{1}-x_{3}\right), p_{1} p_{3} \widehat{m}^{d}+p_{1} q_{3} \widehat{m}^{c}+q_{1} p_{3} \widehat{m}^{c}\right] \Psi\right\rangle\right| \\
\leq & C N^{3} \mid\left\langle\Psi, V_{N}\left(x_{1}-x_{3}\right) g_{\mu}\left(x_{1}-x_{2}\right) p_{2} \mathbb{1}_{\operatorname{supp}\left(V_{N}\right)}\left(x_{1}-x_{3}\right)\right. \\
& \left.\left(p_{1} p_{3} \widehat{m}^{d}+p_{1} q_{3} \widehat{m}^{c}+q_{1} p_{3} \widehat{m}^{c}\right) \Psi\right\rangle \mid \\
& +C N^{3}\left|\left\langle\Psi, g_{\mu}\left(x_{1}-x_{2}\right) p_{2}\left(p_{1} p_{3} \widehat{m}^{d}+p_{1} q_{3} \widehat{m}^{c}+q_{1} p_{3} \widehat{m}^{c}\right) V_{N}\left(x_{1}-x_{3}\right) \Psi\right\rangle\right| .
\end{aligned}
$$

Both lines are bounded by

$$
\begin{aligned}
& C N^{3}\left\|V_{N}\left(x_{1}-x_{3}\right) \Psi\right\|\left\|g_{\mu}\left(x_{1}-x_{2}\right) p_{2}\right\|_{\text {op }} \\
& \quad \times\left(2\left\|\mathbb{1}_{\operatorname{supp}\left(V_{N}\right)}\left(x_{1}-x_{3}\right) p_{1}\right\|_{\text {op }}+\left\|\mathbb{1}_{\operatorname{supp}\left(V_{N}\right)}\left(x_{1}-x_{3}\right) p_{3}\right\|_{\text {op }}\right)\left(\left\|\widehat{m}^{d}\right\|_{\text {op }}+\left\|\widehat{m}^{c}\right\|_{\text {op }}\right) .
\end{aligned}
$$


In view of Lemmas $4.2(\mathrm{e})$ and $5.5(\mathrm{i}),\left\|g_{\mu}\left(x_{1}-x_{2}\right) p_{2}\right\|_{\text {op }} \leq\|\varphi\|_{\infty}\left\|g_{\mu}\right\| \leq C\|\varphi\|_{\infty} N^{-1-\mu}$ $\ln (N)$. Using (111), together with $\left\|\mathbb{1}_{\operatorname{supp}\left(V_{N}\right)}\left(x_{1}-x_{3}\right) p_{1}\right\|_{\text {op }}\left\|V_{N}\left(x_{1}-x_{3}\right) \Psi\right\| \leq \mathcal{K}\left(\varphi, A_{t}\right)$ $N^{-1 / 2}$, we obtain, using $\xi<1 / 2$,

$$
(112) \leq \mathcal{K}\left(\varphi, A_{t}\right) N^{-1 / 2+3 \xi-\mu} \ln (N) \leq \mathcal{K}\left(\varphi, A_{t}\right) N^{1 / 2+\xi-\mu} \ln (N) .
$$

We continue with

$$
\begin{aligned}
(113)+(114)+(115) \leq & C N^{3}\left\|V_{N}\left(x_{1}-x_{3}\right) \Psi\right\|\left\|g_{\mu}\left(x_{1}-x_{2}\right) p_{2}\right\|_{\mathrm{op}} \\
& \times\left\|\mathbb{1}_{\operatorname{supp}\left(V_{N}\right)}\left(x_{1}-x_{3}\right) p_{1}\right\|_{\mathrm{op}}\left\|\left(\widehat{m}^{b}-2 \widehat{m}^{a}\right)\right\|_{\mathrm{op}} \\
& +C N^{3}\left\|g_{\mu}\left(x_{1}-x_{2}\right) p_{2}\right\|_{\mathrm{op}}\left\|\widehat{m}^{b}-2 \widehat{m}^{a}\right\|_{\mathrm{op}}\left\|p_{1} V_{N}\left(x_{1}-x_{3}\right) \Psi\right\| \\
& +C N^{3}\left\|g_{\mu}\left(x_{1}-x_{2}\right) p_{1}\right\|_{\mathrm{op}}\left\|\widehat{m}^{a}\right\|_{\mathrm{op}}\left\|p_{1} V_{N}\left(x_{1}-x_{3}\right) \Psi\right\| \\
\leq & \mathcal{K}\left(\varphi, A_{t}\right) N^{1 / 2+\xi-\mu} \ln (N) .
\end{aligned}
$$

Next, we estimate (116). The support of the function $g_{\mu}\left(x_{1}-x_{2}\right) V_{N}\left(x_{1}-x_{3}\right)$ is such that $\left|x_{1}-x_{2}\right| \leq C N^{-\mu}$, as well as $\left|x_{1}-x_{3}\right| \leq C e^{-N}$. Therefore, $g_{\mu}\left(x_{1}-x_{2}\right) V_{N}\left(x_{1}-\right.$ $\left.x_{3}\right) \neq 0$ implies $\left|x_{2}-x_{3}\right| \leq C N^{-\mu}$. We estimate

$$
\begin{aligned}
(116)= & C N^{3}\left|\left\langle\Psi, g_{\mu}\left(x_{1}-x_{2}\right) V_{N}\left(x_{1}-x_{3}\right) p_{1} \mathbb{1}_{B_{C N^{-\mu}}(0)}\left(x_{2}-x_{3}\right) \widehat{m}^{a} \Psi\right\rangle\right| \\
\leq & C N^{3}\left\|p_{1} V_{N}\left(x_{1}-x_{3}\right) g_{\mu}\left(x_{1}-x_{2}\right) \Psi\right\|\left\|\mathbb{1}_{B_{C N^{-\mu}}(0)}\left(x_{2}-x_{3}\right) \widehat{m}^{a} \Psi\right\| \\
\leq & C N^{3}\left\|p_{1} \mathbb{1}_{\operatorname{supp}\left(V_{N}\right)}\left(x_{1}-x_{3}\right)\right\|_{\mathrm{op}}\left\|g_{\mu}\left(x_{1}-x_{2}\right) V_{N}\left(x_{1}-x_{3}\right) \Psi\right\| \\
& \times\left\|\mathbb{1}_{B_{C N^{-\mu}}(0)}\left(x_{2}-x_{3}\right) \widehat{m}^{a} \Psi\right\| \\
\leq & C_{p} N^{5 / 2}\left\|g_{\mu}\right\|_{\infty}\left\|\mathbb{1}_{B_{C N^{-\mu}}(0)}\right\|_{\frac{p}{p-1}}^{\frac{1}{2}}\left\|\nabla_{1} \widehat{m}^{a} \Psi\right\|^{\frac{p-1}{p}}\left\|\widehat{m}^{a} \Psi\right\|^{\frac{1}{p}} \\
\leq & C N^{5 / 2}\left\|g_{\mu}\right\|_{\infty} N^{-\mu / 2}\left\|\nabla_{1} \widehat{m}^{a} \Psi\right\|^{1 / 2}\left\|\widehat{m}^{a} \Psi\right\|^{1 / 2} \\
\leq & C N^{3 / 2+\xi-\mu / 2} .
\end{aligned}
$$

In the fourth line, we applied Sobolev inequality as in the proof of Lemma 7.4, then setting $p=2$. Furthermore, we used $\left\|\nabla_{1} \widehat{m}^{a} \Psi\right\|^{1 / 2}\left\|\widehat{m}^{a} \Psi\right\|^{1 / 2} \leq C N^{-1+\xi}$, as well as $\left\|g_{\mu}\right\|_{\infty} \leq C$, see Lemma 5.5.

Using Lemma 4.2 (d), (117) can be bounded by

$$
\begin{aligned}
& C N^{3}\left|\left\langle\Psi, g_{\mu}\left(x_{1}-x_{2}\right)\left[4 \pi|\varphi|^{2}\left(x_{3}\right), p_{1} p_{2}\left(\widehat{r}-\widehat{r}_{2}\right)+\left(p_{1} q_{2}+q_{1} p_{2}\right)\left(\widehat{r}-\widehat{r}_{1}\right)\right] \Psi\right\rangle\right| \\
& \quad \leq C N^{3}\|\varphi\|_{\infty}^{2}\left(\left\|\widehat{r}-\widehat{r}_{2}\right\|_{\text {op }}+\left\|\widehat{r}-\widehat{r}_{1}\right\|_{\text {op }}\right)\left\|g_{\mu}\left(x_{1}-x_{2}\right) p_{2}\right\|_{\text {op }} .
\end{aligned}
$$

Note that $\left\|\widehat{r}-\widehat{r}_{2}\right\|_{\text {op }}+\left\|\widehat{r}-\widehat{r}_{1}\right\|_{\text {op }} \leq \sum_{j \in\{c, d, e, f\}}\left\|\widehat{m}^{j}\right\|_{\text {op }} \leq C N^{-2+3 \xi}$ holds. With $\left\|g_{\mu}\left(x_{1}-x_{2}\right) p_{2}\right\|_{\text {op }} \leq C N^{-1-\mu} \ln (N)$, it then follows that

$$
|(117)| \leq C\|\varphi\|_{\infty}^{2} N^{3 \xi-\mu} \ln (N) .
$$

In total, we obtain

$$
\left|\gamma_{d}(\Psi, \varphi)\right| \leq \mathcal{K}\left(\varphi, A_{t}\right)\left(N^{3 / 2+\xi-\mu / 2}+N^{1 / 2+3 \xi-\mu} \ln (N)\right) .
$$

\section{Control of $\gamma_{e}$}


Recall that

$$
\gamma_{e}(\Psi, \varphi)=-\frac{1}{2} N(N-1)(N-2)(N-3) \Im\left(\left\langle\Psi, g_{\mu}\left(x_{1}-x_{2}\right)\left[V_{N}\left(x_{3}-x_{4}\right), \widehat{r}\right] \Psi\right\rangle\right) \text {. }
$$

Using symmetry, Lemma 4.2 (d) and notation (110), $\gamma_{e}$ is bounded by

$$
\begin{aligned}
\gamma_{e}(\Psi, \varphi) \leq & N^{4} \mid\left\langle\Psi \Psi, g_{\mu}\left(x_{1}-x_{2}\right)\left[V_{N}\left(x_{3}-x_{4}\right), \widehat{m}^{c} p_{1} p_{2} p_{3} p_{4}+2 \widehat{m}^{d} p_{1} p_{2} p_{3} q_{4}\right.\right. \\
& \left.\left.+2 \widehat{m}^{e} p_{1} q_{2} p_{3} p_{4}+4 \widehat{m}^{f} p_{1} q_{2} p_{3} q_{4}\right] \Psi\right\rangle \mid \\
\leq & 4 N^{4}\left\|V_{N}\left(x_{3}-x_{4}\right) \Psi\right\|\left\|\mathbb{1}_{\mathrm{supp}\left(V_{N}\right)}\left(x_{3}-x_{4}\right) p_{3}\right\|_{\mathrm{op}}\left\|g_{\mu}\left(x_{1}-x_{2}\right) p_{1}\right\|_{\mathrm{op}} \\
& \times\left(\left\|\widehat{m}^{c}\right\|_{\mathrm{op}}+\left\|\widehat{m}^{d}\right\|_{\mathrm{op}}+\left\|\widehat{m}^{e}\right\|_{\mathrm{op}}+\left\|\widehat{m}^{f}\right\|_{\mathrm{op}}\right) .
\end{aligned}
$$

We get with (111), Lemma 5.5 and Lemma 4.2 that

$$
\left|\gamma_{e}(\Psi, \varphi)\right| \leq \mathcal{K}\left(\varphi, A_{t}\right) N^{1 / 2+3 \xi-\mu} \ln (N) .
$$

\section{Control of $\gamma_{f}$}

Recall that

$$
\gamma_{f}(\Psi, \varphi)=2 N(N-1) \frac{N-2}{N-1} \Im\left(\left\langle\Psi, g_{\mu}\left(x_{1}-x_{2}\right)\left[4 \pi|\varphi|^{2}\left(x_{1}\right), \widehat{r}\right] \Psi\right\rangle\right) .
$$

We obtain the estimate

$$
\left|\gamma_{f}(\Psi, \varphi)\right| \leq \mathcal{K}\left(\varphi, A_{t}\right) N^{2}\left\|g_{\mu}\right\|\|\widehat{r}\|_{\text {op }} \leq \mathcal{K}\left(\varphi, A_{t}\right) N^{\xi-\mu} \ln (N) .
$$

Proof of Lemma 6.13. Let the assumptions of Lemma 6.13 be satisfied. With the previous estimates and $\xi<1 / 3$ we get

$$
\begin{aligned}
& \quad \sum_{k \in\{a, b, c, d, e, f\}}\left|\gamma_{k}\left(\Psi_{t}, \varphi_{t}\right)\right| \leq \mathcal{K}\left(\varphi_{t}, A_{t}\right)\left(\alpha\left(\Psi_{t}, \varphi_{t}\right)+N^{-\xi}+\left(N^{2-\mu / 2}+N^{-1 / 6}\right) \ln (N)\right. \\
& \left.+\inf _{\min \{\beta, 1 / 2\}>\beta_{1}>0} \inf _{\eta>0}\left(N^{\eta-2 \beta_{1}} \ln (N)^{2}+N^{-1+2 \beta_{1}+\xi}+N^{2 \xi-\eta}\right)\right) .
\end{aligned}
$$

Choosing $\xi=1 / 10, \mu=10, \eta=3 / 10$ and $\beta_{1}=3 / 10$, we obtain (64).

7.5. Energy estimates. In this section we show that $\left\|\mathbb{1}_{\mathcal{A}_{1}^{(d)}} \nabla_{1} q_{1} \Psi\right\|^{2}$ can be controlled sufficiently well in terms of the counting functionals $\alpha^{<}$and $\alpha$. If $\Psi_{t}$ is evolving according to $W_{\beta}$, one could actually show that $\left\|\nabla_{1} q_{1} \Psi_{t}\right\|^{2}$ is small already without cutoff. While such a proof would be less involved, we chose a unified presentation which both covers the Gross-Pitaevskii scaling and the NLS scaling.

Lemma 7.9. Let $W_{\beta} \in \mathcal{W}_{\beta}, V_{N} \in \mathcal{V}_{N}$ and $A_{t} \in L^{\infty}\left(\mathbb{R}^{2}, \mathbb{R}\right)$. Let $\Psi \in L_{s}^{2}\left(\mathbb{R}^{2 N}, \mathbb{C}\right) \cap$ $H^{1}\left(\mathbb{R}^{2 N}, \mathbb{C}\right),\|\Psi\|=1$ with $\left\|\nabla_{1} \Psi\right\| \leq C$. Let $\varphi \in H^{3}\left(\mathbb{R}^{2}, \mathbb{C}\right),\|\varphi\|=1$. For $d \geq 3$, define the sets $\mathcal{A}_{1}^{(d)}, \overline{\mathcal{B}}_{1}^{(d)}$ as in Definition 7.3. Then, for $N$ large enough and $d \geq 3$,

$$
\begin{aligned}
\left\|\mathbb{1}_{\mathcal{A}_{1}^{(d)}} \nabla_{1} q_{1} \Psi\right\|^{2} \leq & \mathcal{K}\left(\varphi, A_{t}\right)\left(\left\langle\Psi, \widehat{n}^{\varphi} \Psi\right\rangle+N^{-1 / 6} \ln (N)\right. \\
& \left.+\inf \left\{\left|\mathcal{E}_{V_{N}}(\Psi)-\mathcal{E}_{4 \pi}^{G P}(\varphi)\right|,\left|\mathcal{E}_{W_{\beta}}(\Psi)-\mathcal{E}_{b_{W_{\beta}}}^{G P}(\varphi)\right|+N^{-2 \beta} \ln (N)\right\}\right) .
\end{aligned}
$$


Proof. We start with expanding $\mathcal{E}_{W_{\beta}}(\Psi)-\mathcal{E}_{N\left\|W_{\beta}\right\|_{1}}^{G P}(\varphi)$. This yields

$$
\begin{aligned}
\mathcal{E}_{W_{\beta}}(\Psi)-\mathcal{E}_{N\left\|W_{\beta}\right\|_{1}}^{G P}(\varphi) & =\left\|\nabla_{1} \Psi\right\|^{2}+\frac{N-1}{2}\left\|\sqrt{W_{\beta}}\left(x_{1}-x_{2}\right) \Psi\right\|^{2} \\
& -\|\nabla \varphi\|^{2}-\frac{1}{2} N\left\|W_{\beta}\right\|_{1}\left\|\varphi^{2}\right\|^{2}+\left\langle\Psi, A_{t}\left(x_{1}\right) \Psi\right\rangle-\left\langle\varphi, A_{t} \varphi\right\rangle \\
& =\left\|\mathbb{1}_{\mathcal{A}_{1}^{(d)}} \nabla_{1} q_{1} \Psi\right\|^{2}+M(\Psi, \varphi)+Q_{\beta}(\Psi, \varphi),
\end{aligned}
$$

where we have defined

$$
\begin{aligned}
M(\Psi, \varphi)= & 2 \Re\left(\left\langle\nabla_{1} q_{1} \Psi, \mathbb{1}_{\mathcal{A}_{1}^{(d)}} \nabla_{1} p_{1} \Psi\right\rangle\right) \\
& +\left\|\mathbb{1}_{\mathcal{A}_{1}^{(d)}} \nabla_{1} p_{1} \Psi\right\|^{2}-\|\nabla \varphi\|^{2} \\
& +\left\langle\Psi \Psi, A_{t}\left(x_{1}\right) \Psi\right\rangle-\left\langle\varphi, A_{t} \varphi\right\rangle, \\
Q_{\beta}(\Psi, \varphi)= & \left\|\mathbb{1}_{\mathcal{A}_{1}^{(d)}} \nabla_{1} \Psi\right\|^{2} \\
& +\frac{N-1}{2}\left\langle\Psi,\left(1-p_{1} p_{2}\right) W_{\beta}\left(x_{1}-x_{2}\right)\left(1-p_{1} p_{2}\right) \Psi\right\rangle \\
& +\frac{N-1}{2}\left\langle\Psi, p_{1} p_{2} W_{\beta}\left(x_{1}-x_{2}\right) p_{1} p_{2} \Psi\right\rangle-\frac{1}{2} N\left\|W_{\beta}\right\|_{1}\left\|\varphi^{2}\right\|^{2} \\
& +(N-1) \Re\left\langle\Psi \Psi,\left(1-p_{1} p_{2}\right) W_{\beta}\left(x_{1}-x_{2}\right) p_{1} p_{2} \Psi\right\rangle .
\end{aligned}
$$

Notice that the first two terms in $Q_{\beta}(\Psi, \varphi)$ are nonnegative. This yields to the bound

$$
\begin{aligned}
S_{\beta}(\Psi, \varphi)= & (N-1)\left|\left\langle\Psi,\left(1-p_{1} p_{2}\right) W_{\beta}\left(x_{1}-x_{2}\right) p_{1} p_{2} \Psi\right\rangle\right| \\
& +\mid \frac{N-1}{2}\left\langle\left\langle\Psi, p_{1} p_{2} W_{\beta}\left(x_{1}-x_{2}\right) p_{1} p_{2} \Psi\right\rangle-\frac{1}{2} N\left\|W_{\beta}\right\|_{1}\left\|\varphi^{2}\right\|^{2}\right| \\
\geq & -Q_{\beta}(\Psi, \varphi) .
\end{aligned}
$$

We therefore obtain

$$
\left\|\mathbb{1}_{\mathcal{A}_{1}^{(d)}} \nabla_{1} q_{1} \Psi\right\|^{2} \leq\left|\mathcal{E}_{W_{\beta}}(\Psi)-\mathcal{E}_{N\left\|W_{\beta}\right\|_{1}}^{G P}(\varphi)\right|+|M(\Psi, \varphi)|+\left|S_{\beta}(\Psi, \varphi)\right| .
$$

Thus if we use that Definition 2.1 implies the estimate

$$
\left|\mathcal{E}_{b_{W_{\beta}}}^{G P}(\varphi)-\mathcal{E}_{N\left\|W_{\beta}\right\|_{1}}^{G P}(\varphi)\right| \leq \frac{1}{2}\left|b_{W_{\beta}}-N\left\|W_{\beta}\right\|_{1}\right|\left\|\varphi^{2}\right\|^{2} \leq \mathcal{K}\left(\varphi, A_{t}\right) N^{-1} \ln (N),
$$

we get the bound:

$$
\begin{aligned}
\left\|\mathbb{1}_{\mathcal{A}_{1}^{(d)}} \nabla_{1} q_{1} \Psi\right\|^{2} \leq & \left|\mathcal{E}_{W_{\beta}}(\Psi)-\mathcal{E}_{b_{W_{\beta}} P}(\varphi)\right|+\mathcal{K}\left(\varphi, A_{t}\right) N^{-1} \ln (N) \\
& +|M(\Psi, \varphi)|+\left|S_{\beta}(\Psi, \varphi)\right|
\end{aligned}
$$

Next, we split up the energy difference $\mathcal{E}_{V_{N}}(\Psi)-\mathcal{E}_{4 \pi}^{G P}(\varphi)$,

$$
\begin{aligned}
\mathcal{E}_{V_{N}}(\Psi)-\mathcal{E}_{4 \pi}^{G P}(\varphi)= & \left\|\nabla_{1} \Psi\right\|^{2}+\frac{N-1}{2}\left\|\sqrt{V_{N}}\left(x_{1}-x_{2}\right) \Psi\right\|^{2}-\|\nabla \varphi\|^{2} \\
& -2 \pi\left\|\varphi^{2}\right\|^{2}+\left\langle\left\langle\Psi, A_{t}\left(x_{1}\right) \Psi\right\rangle-\left\langle\varphi, A_{t} \varphi\right\rangle .\right.
\end{aligned}
$$


In order to better estimate the terms corresponding to the two-particle interactions, we introduce, for $v>d$, the potential $M_{v}(x)$, defined in Definition 5.3. Note, that $v>d$ assures that that part of the interaction $M_{v}$ which lies within the set $\mathcal{A}_{1}^{(d)}$ will be negligible. We continue with

$$
\begin{aligned}
\mathcal{E}_{V_{N}}(\Psi)-\mathcal{E}_{4 \pi}^{G P}(\varphi)= & \left\|\mathbb{1}_{\mathcal{A}_{1}^{(d)}} \nabla_{1} \Psi\right\|^{2}+\left\|\mathbb{1}_{\overline{\mathcal{A}}_{1}^{(d)}} \nabla_{1} \Psi\right\|^{2} \\
& +\frac{N-1}{2}\left\|\mathbb{1}_{\overline{\mathcal{B}}_{1}^{(d)}} \sqrt{V_{N}}\left(x_{1}-x_{2}\right) \Psi\right\|^{2} \\
& +\frac{1}{2}\left\langle\Psi \Psi, \sum_{j \neq 1} \mathbb{1}_{\mathcal{B}_{1}^{(d)}}\left(V_{N}-M_{\nu}\right)\left(x_{1}-x_{j}\right) \Psi\right\rangle \\
& +\frac{1}{2}\left\langle\left\langle\Psi, \sum_{j \neq 1} \mathbb{1}_{\mathcal{B}_{1}^{(d)}} M_{\nu}\left(x_{1}-x_{j}\right) \Psi\right\rangle-\|\nabla \varphi\|^{2}-2 \pi\left\|\varphi^{2}\right\|^{2}\right. \\
& +\left\langle\Psi, A_{t}\left(x_{1}\right) \Psi\right\rangle-\left\langle\varphi, A_{t} \varphi\right\rangle .
\end{aligned}
$$

After reordering, the identity $q_{1}=1-p_{1}$, together with the symmetry of $\Psi \in$ $L_{s}^{2}\left(\mathbb{R}^{2 N}, \mathbb{C}\right)$ gives

$$
\begin{aligned}
\mathcal{E}_{V_{N}}(\Psi)-\mathcal{E}_{4 \pi}^{G P}(\varphi)= & \left\|\mathbb{1}_{\mathcal{A}_{1}^{(d)}} \nabla_{1} q_{1} \Psi\right\|^{2}+\left\|\mathbb{1}_{\overline{\mathcal{B}}_{1}^{(d)}} \mathbb{1}_{\mathcal{A}_{1}^{(d)}} \nabla_{1} \Psi\right\|^{2} \\
& +\frac{N-1}{2}\left\|\mathbb{1}_{\overline{\mathcal{B}}_{1}^{(d)}} \sqrt{V_{N}}\left(x_{1}-x_{2}\right) \Psi\right\|^{2} \\
& +\frac{N-1}{2}\left\langle\Psi, \mathbb{1}_{\mathcal{B}_{1}^{(d)}}\left(1-p_{1} p_{2}\right) M_{\nu}\left(x_{1}-x_{2}\right)\left(1-p_{1} p_{2}\right) \mathbb{1}_{\mathcal{B}_{1}^{(d)}} \Psi\right\rangle \\
& +\left\|\mathbb{1}_{\mathcal{B}_{1}^{(d)}} \mathbb{1}_{\mathcal{A}_{1}}^{(d)} \nabla_{1} \Psi\right\|^{2}+\frac{1}{2}\left\langle\Psi \Psi, \sum_{j \neq 1} \mathbb{1}_{\mathcal{B}_{1}^{(d)}}\left(V_{N}-M_{\nu}\right)\left(x_{1}-x_{j}\right) \Psi\right\rangle \\
& +\frac{N-1}{2}\left\langle\left\langle\Psi, \mathbb{1}_{\mathcal{B}_{1}^{(d)}} p_{1} p_{2} M_{\nu}\left(x_{1}-x_{2}\right) p_{1} p_{2} \mathbb{1}_{\mathcal{B}_{1}^{(d)}} \Psi\right\rangle-2 \pi\left\|\varphi^{2}\right\|^{2}\right. \\
& +2 \Re\left(\left\langle\nabla_{1} q_{1} \Psi, \mathbb{1}_{\mathcal{A}_{1}^{(d)}} \nabla_{1} p_{1} \Psi\right\rangle\right) \\
& +(N-1) \Re\left\langle\Psi, \mathbb{1}_{\mathcal{B}_{1}^{(d)}}\left(1-p_{1} p_{2}\right) M_{\nu}\left(x_{1}-x_{2}\right) p_{1} p_{2} \mathbb{1}_{\left.\mathcal{B}_{1}^{(d)} \Psi\right\rangle}\right. \\
& +\left\|\mathbb{1}_{\mathcal{A}_{1}^{(d)}} \nabla_{1} p_{1} \Psi\right\|^{2}-\|\nabla \varphi\|^{2} \\
& +\left\langle\Psi, A_{t}\left(x_{1}\right) \Psi\right\rangle-\left\langle\varphi, A_{t} \varphi\right\rangle \\
= & \| \mathbb{1}_{\mathcal{A}_{1}^{(d)} \nabla_{1} q_{1} \Psi \|^{2}+M(\Psi, \varphi)+\tilde{Q}_{\nu}(\Psi, \varphi) .}
\end{aligned}
$$

with

$$
\begin{aligned}
\tilde{Q}_{\nu}(\Psi, \varphi)= & \left\|\mathbb{1}_{\overline{\mathcal{B}}_{1}^{(d)}} \mathbb{1}_{\overline{\mathcal{A}}_{1}^{(d)}} \nabla_{1} \Psi\right\|^{2} \\
& +\frac{N-1}{2}\left\langle\Psi \Psi, \mathbb{1}_{\mathcal{B}_{1}^{(d)}}\left(1-p_{1} p_{2}\right) M_{\nu}\left(x_{1}-x_{2}\right)\left(1-p_{1} p_{2}\right) \mathbb{1}_{\mathcal{B}_{1}^{(d)}} \Psi\right\rangle \\
& +\frac{N-1}{2}\left\|\mathbb{1}_{\overline{\mathcal{B}}_{1}^{(d)}} \sqrt{V_{N}}\left(x_{1}-x_{2}\right) \Psi\right\|^{2} \\
& +\left\|\mathbb{1}_{\mathcal{B}_{1}^{(d)}} \mathbb{1}_{\overline{\mathcal{A}}_{1}^{(d)}} \nabla_{1} \Psi\right\|^{2}+\frac{1}{2}\left\langle\Psi, \sum_{j \neq 1} \mathbb{1}_{\mathcal{B}_{1}^{(d)}}\left(V_{N}-M_{\nu}\right)\left(x_{1}-x_{j}\right) \Psi\right\rangle
\end{aligned}
$$




$$
\begin{aligned}
& +(N-1) \Re\left\langle\Psi \Psi, \mathbb{1}_{\mathcal{B}_{1}^{(d)}}\left(1-p_{1} p_{2}\right) M_{v}\left(x_{1}-x_{2}\right) p_{1} p_{2} \mathbb{1}_{\mathcal{B}_{1}^{(d)}} \Psi\right\rangle \\
& +\frac{N-1}{2}\left\langle\Psi \Psi, \mathbb{1}_{\mathcal{B}_{1}^{(d)}} p_{1} p_{2} M_{\nu}\left(x_{1}-x_{2}\right) p_{1} p_{2} \mathbb{1}_{\mathcal{B}_{1}^{(d)}} \Psi\right\rangle-2 \pi\left\|\varphi^{2}\right\|^{2} .
\end{aligned}
$$

The first three terms in $\tilde{Q}_{v}(\Psi, \varphi)$ are nonnegative. For $v>d$ and $N$ large enough, Lemma 7.10 implies that (124) is also nonnegative. Thus, for $v>d$, we obtain the bound

$$
\begin{aligned}
\tilde{S}_{v}(\Psi, \varphi)= & (N-1)\left|\left\langle\Psi, \mathbb{1}_{\mathcal{B}_{1}^{(d)}}\left(1-p_{1} p_{2}\right) M_{\nu}\left(x_{1}-x_{2}\right) p_{1} p_{2} \mathbb{1}_{\mathcal{B}_{1}^{(d)}} \Psi\right\rangle\right| \\
& +\left|\frac{N-1}{2}\left\langle\Psi \Psi, \mathbb{1}_{\mathcal{B}_{1}^{(d)}} p_{1} p_{2} M_{\nu}\left(x_{1}-x_{2}\right) p_{1} p_{2} \mathbb{1}_{\mathcal{B}_{1}^{(d)}} \Psi\right\rangle-2 \pi\left\|\varphi^{2}\right\|^{2}\right| \\
\geq & -\tilde{Q}_{\nu}(\Psi, \varphi) .
\end{aligned}
$$

In total, we obtain

$$
\left\|\mathbb{1}_{\mathcal{A}_{1}^{(d)}} \nabla_{1} q_{1} \Psi\right\|^{2} \leq|M(\Psi, \varphi)|+\tilde{S}_{\nu}(\Psi, \varphi)+\left|\mathcal{E}_{V_{N}}(\Psi)-\mathcal{E}_{4 \pi}^{G P}(\varphi)\right| .
$$

It is therefore left to estimate $M(\Psi, \varphi), S_{\beta}(\Psi, \varphi)$ and $\tilde{S}_{v}(\Psi, \varphi)$.

Estimate of $S_{\beta}(\Psi, \varphi)$ and $\tilde{S}_{v}(\Psi, \varphi)$.

The contributions (121) and (125) are estimated in Lemma 7.11.

$$
\text { (121), (125) } \leq \mathcal{K}\left(\varphi, A_{t}\right)\left(\langle\Psi, \widehat{n} \Psi\rangle+N^{-1 / 6} \ln (N)\right) .
$$

We are thus left to estimate (122) and (126). We begin with the estimate for (126). As in (80), we can write

$$
\left\langle\Psi, \mathbb{1}_{\mathcal{B}_{1}^{(d)}} p_{1} p_{2} M_{v}\left(x_{1}-x_{2}\right) p_{1} p_{2} \mathbb{1}_{\mathcal{B}_{1}^{(d)}} \Psi\right\rangle=\left\langle\varphi, M_{v} *|\varphi|^{2} \varphi\right\rangle\left\langle\Psi \Psi, \mathbb{1}_{\mathcal{B}_{1}^{(d)}} p_{1} p_{2} \mathbb{1}_{\mathcal{B}_{1}^{(d)}} \Psi\right\rangle .
$$

With Lemma 7.3 (c) with $\epsilon=1 / 2$, we get $\left\|\mathbb{1}_{\overline{\mathcal{B}}_{1}^{(d)}} \Psi\right\| \leq C N^{3 / 2-d}$. Together with $\left\|p_{1} p_{2} \Psi\right\|^{2}=1+2\left\|p_{1} q_{2} \Psi\right\|^{2}+\left\|q_{1} q_{2} \Psi\right\|^{2}$, we therefore obtain

$$
\begin{aligned}
(126) \leq & 3\left\|q_{1} \Psi\right\|^{2}+C\left(N^{3 / 2-d}+N^{3-2 d}\right)+\frac{1}{2}\left|N\left\langle\varphi, M_{v} *|\varphi|^{2} \varphi\right\rangle-N\left\|M_{\nu}\right\|_{1}\left\|\varphi^{2}\right\|^{2}\right| \\
& +\frac{1}{2}\left|4 \pi-N\left\|M_{\nu}\right\|_{1}\right|\left\|\varphi^{2}\right\|^{2}+\frac{1}{2}\left\langle\varphi, M_{\nu} *|\varphi|^{2} \varphi\right\rangle .
\end{aligned}
$$

Note that, using Young's inequality and (80)

$$
\begin{aligned}
\mid\langle\varphi & \left., N M_{v} *|\varphi|^{2} \varphi\right\rangle-N\left\|M_{v}\right\|_{1}\left\|\varphi^{2}\right\|^{2} \mid \\
& =\left.\left|\int_{\mathbb{R}^{2}} d^{2} x\right| \varphi(x)\right|^{2}\left(N\left(M_{v} *|\varphi|^{2}\right)(x)-N\left\|M_{v}\right\|_{1}|\varphi(x)|^{2}\right) \mid \\
& \leq\|\varphi\|_{\infty}^{2}\left\|N\left(M_{v} *|\varphi|^{2}\right)-\right\| N M_{v}\left\|_{1}|\varphi|^{2}\right\|_{1} \\
& \leq C\|\varphi\|_{\infty}^{2}\left\|\Delta|\varphi|^{2}\right\|_{1} N^{-2 v} \ln (N) \\
& \leq \mathcal{K}\left(\varphi, A_{t}\right) N^{-2 v} \ln (N) .
\end{aligned}
$$


Since $\left|N\left\|M_{\nu}\right\|_{1}-4 \pi\right| \leq C \frac{\ln (N)}{N}$ (see Lemma 5.5) and $\left\langle\varphi, M_{\nu} *|\varphi|^{2} \varphi\right\rangle \leq\|\varphi\|_{\infty}^{4}\left\|M_{\nu}\right\|_{1} \leq$ $C\|\varphi\|_{\infty}^{4} N^{-1}$, it follows that

$$
\begin{aligned}
|(126)| & \leq \mathcal{K}\left(\varphi, A_{t}\right)\left(\left\langle\Psi, \widehat{n}^{\varphi} \Psi\right\rangle+N^{3 / 2-d}+N^{3-2 d+2}+N^{-2 v} \ln (N)+N^{-1} \ln (N)\right) \\
& \leq \mathcal{K}\left(\varphi, A_{t}\right)\left(\left\langle\Psi, \widehat{n}^{\varphi} \Psi\right\rangle+N^{-1} \ln (N)\right)
\end{aligned}
$$

where $v>d \geq 3$ was used in the last inequality.

Using the same estimates, we obtain

$$
(122) \leq \mathcal{K}\left(\varphi, A_{t}\right)\left(\left\langle\Psi, \widehat{n}^{\varphi} \Psi\right\rangle+N^{-2 \beta} \ln (N)+N^{-1} \ln (N)\right) .
$$

In total, we obtain, for any $v>d \geq 1$, the bound

$$
\begin{aligned}
& S_{\beta}(\Psi, \varphi) \leq \mathcal{K}\left(\varphi, A_{t}\right)\left(\langle\Psi, \widehat{n} \Psi\rangle+N^{-2 \beta} \ln (N)+N^{-1 / 6} \ln (N)\right) \\
& \tilde{S}_{\nu}(\Psi, \varphi) \leq \mathcal{K}\left(\varphi, A_{t}\right)\left(\langle\Psi, \widehat{n} \Psi\rangle+N^{-1 / 6} \ln (N)\right) .
\end{aligned}
$$

Estimate of $M(\Psi, \varphi)$. We need to estimate (118), (119) and (120). We start with

$$
\begin{aligned}
|(118)| & \leq 2\left|\left\langle\nabla_{1} q_{1} \Psi, \mathbb{1}_{\overline{\mathcal{A}}_{1}^{(d)}} \nabla_{1} p_{1} \Psi\right\rangle\right|+2\left|\left\langle\nabla_{1} q_{1} \Psi, \nabla_{1} p_{1} \Psi\right\rangle\right| \\
& \leq 2\left\|\nabla_{1} q_{1} \Psi\right\|\left\|\mathbb{1}_{\overline{\mathcal{A}}_{1}^{(d)}} \nabla_{1} p_{1}\right\|_{\mathrm{op}}+2\left|\left\langle\widehat{n}^{-1 / 2} q_{1} \Psi, \Delta_{1} p_{1} \widehat{n}_{1}^{1 / 2} \Psi\right\rangle\right|
\end{aligned}
$$

By Lemma 7.4, we obtain $\left\|\mathbb{1}_{\overline{\mathcal{A}}_{1}^{(d)}} \nabla_{1} p_{1}\right\|_{\text {op }} \leq C\|\nabla \varphi\|_{\infty} N^{1 / 2-d}$.

Furthermore, we use $\left\|\nabla_{1} q_{1} \Psi\right\| \leq\left\|\nabla_{1} \Psi\right\|+\left\|\nabla_{1} p_{1} \Psi\right\| \leq \mathcal{K}\left(\varphi, A_{t}\right)$ (see also Lemma 7.6) and $\left|\left\langle\widehat{n}^{-1 / 2} q_{1} \Psi, \Delta_{1} p_{1} \widehat{n}_{1}^{1 / 2} \Psi\right\rangle\right| \leq \mathcal{K}\left(\varphi, A_{t}\right)\left\|\widehat{n}_{1}^{1 / 2} \Psi\right\|\left\|\widehat{n}^{1 / 2} \Psi\right\| \leq \mathcal{K}\left(\varphi, A_{t}\right)(\langle\Psi \Psi, \widehat{n} \Psi\rangle+$ $\left.N^{-1}\right)$. Hence, for $d \geq 3$,

$$
|(118)| \leq \mathcal{K}\left(\varphi, A_{t}\right)\left(\langle\Psi \Psi, \widehat{n} \Psi\rangle+N^{1-d}+N^{-1}\right) \leq \mathcal{K}\left(\varphi, A_{t}\right)\left(\langle\Psi, \widehat{n} \Psi\rangle+N^{-1}\right) .
$$

With $\left\|\nabla_{1} p_{1} \Psi\right\|^{2}=\|\nabla \varphi\|^{2}\left\|p_{1} \Psi\right\|^{2}$ line (119) is estimated by

$$
\begin{aligned}
(119) & =\left\|\mathbb{1}_{\mathcal{A}_{1}^{(d)}} \nabla_{1} p_{1} \Psi\right\|^{2}-\|\nabla \varphi\|^{2} \\
& \leq\|\| \nabla_{1} p_{1} \Psi\left\|^{2}-\right\| \nabla \varphi\left\|^{2} \mid+\right\| \mathbb{1}_{\overline{\mathcal{A}}_{1}^{(d)}} \nabla_{1} p_{1} \Psi \|^{2} \\
& \leq C\left(\|\nabla \varphi\|^{2}\left\langle\Psi \Psi, q_{1} \Psi\right\rangle+\|\nabla \varphi\|_{\infty}^{2} N^{1-2 d}\right) \\
& \leq \mathcal{K}\left(\varphi, A_{t}\right)\left(\langle\Psi, \widehat{n} \Psi\rangle+N^{-1}\right) .
\end{aligned}
$$

For line (120), we use Lemma 7.5 to obtain

$$
(120) \leq C\left\|A_{t}\right\|_{\infty}\left(\langle\Psi, \widehat{n} \Psi\rangle+N^{-1 / 2}\right) .
$$

In total, we obtain

$$
M(\Psi, \varphi) \leq \mathcal{K}\left(\varphi, A_{t}\right)\left(\langle\Psi, \widehat{n} \Psi\rangle+N^{-1 / 2}\right) .
$$


Lemma 7.10. (a) Let $V_{N} \in \mathcal{V}_{N}$ and let $R_{v}$ and $M_{v}$ be defined as in Definition 5.3. Then, for any $\Psi \in L^{2}\left(\mathbb{R}^{2 N}, \mathbb{C}\right) \cap \mathcal{D}\left(\nabla_{1}\right)$

$$
\left\|\mathbb{1}_{\left|x_{1}-x_{2}\right| \leq R_{v}} \nabla_{1} \Psi\right\|^{2}+\frac{1}{2}\left\langle\Psi,\left(V_{N}-M_{\nu}\right)\left(x_{1}-x_{2}\right) \Psi\right\rangle \geq 0 .
$$

(b) Let $V_{N} \in \mathcal{V}_{N}$ and let $M_{v}$ be defined as in Definition 5.3. Let $\Psi \in L_{s}^{2}\left(\mathbb{R}^{2 N}, \mathbb{C}\right) \cap$ $H^{1}\left(\mathbb{R}^{2 N}, \mathbb{C}\right)$. Then, for sufficiently large $N$ and for $v>d$,

$$
\left\|\mathbb{1}_{\mathcal{B}_{1}^{(d)}} \mathbb{1}_{\mathcal{A}_{1}^{(d)}} \nabla_{1} \Psi\right\|^{2}+\frac{1}{2}\left\langle\Psi, \sum_{j \neq 1} \mathbb{1}_{\mathcal{B}_{1}^{(d)}}\left(V_{N}-M_{\nu}\right)\left(x_{1}-x_{j}\right) \Psi\right\rangle \geq 0 .
$$

Proof. (a) We first show nonnegativity of the one-particle operator $H^{Z_{n}}: H^{2}\left(\mathbb{R}^{2}, \mathbb{C}\right) \rightarrow$ $L^{2}\left(\mathbb{R}^{2}, \mathbb{C}\right)$ given by

$$
H^{Z_{n}}=-\Delta+\frac{1}{2} \sum_{z_{k} \in Z_{n}}\left(V_{N}\left(\cdot-z_{k}\right)-M_{\nu}\left(\cdot-z_{k}\right)\right)
$$

for any $n \in \mathbb{N}$ and any $n$-elemental subset $Z_{n} \subset \mathbb{R}^{2}$ which is such that the supports of the potentials $M_{v}\left(\cdot-z_{k}\right)$ are pairwise disjoint for any two $z_{k} \in Z_{n}$.

Since $f_{v}\left(\cdot-z_{k}\right)$ is the the zero energy scattering state of the potential $1 / 2 V_{N}(\cdot-$ $\left.z_{k}\right)-1 / 2 M_{v}\left(\cdot-z_{k}\right)$, it follows that

$$
F_{v}^{Z_{n}}=\prod_{z_{k} \in Z_{n}} f_{v}\left(\cdot-z_{k}\right)
$$

fulfills $H^{Z_{n}} F_{v}^{Z_{n}}=0$ for any such $Z_{n}$. By construction $f_{v}$ is a nonnegative function, so is $F_{v}^{Z_{n}}$. Since $\frac{1}{2} \sum_{z_{k} \in Z_{n}}\left(V_{N}\left(\cdot-z_{k}\right)-M_{v}\left(\cdot-z_{k}\right)\right) \in L^{\infty}\left(\mathbb{R}^{2}, \mathbb{C}\right)$, this potential is a infinitesimal perturbation of $-\Delta$, thus $\sigma_{\text {ess }}\left(H^{Z_{n}}\right)=[0, \infty)$. Assume now that $H^{Z_{n}}$ is not nonnegative. Then, there exists a ground state $\Psi_{G} \in H^{2}\left(\mathbb{R}^{2}, \mathbb{C}\right)$ of $H^{Z_{n}}$ of negative energy $E<0$. The phase of the ground state can be chosen such that the ground state is real and positive a.e. (see e.g. [52], Theorem 10.12.). Since $f_{v}\left(\cdot-z_{k}\right)$ is positive outside $\operatorname{supp}\left(V_{N}\right)$, the following inequality is valid 6

$$
\left\langle F_{v}^{Z_{n}}, H^{Z_{n}} \Psi_{G}\right\rangle=\left\langle F_{v}^{Z_{n}}, E \Psi_{G}\right\rangle<0 .
$$

On the other hand we have since $F_{v}^{Z_{n}}$ is the zero energy scattering state

$$
\left\langle F_{v}^{Z_{n}}, H^{Z_{n}} \Psi_{G}\right\rangle=\left\langle H^{Z_{n}} F_{v}^{Z_{n}}, \Psi_{G}\right\rangle=0 .
$$

This contradicts (131) and the nonnegativity of $H^{Z_{n}}$ follows.

Now, assume that there exists a $\psi \in H^{2}\left(\mathbb{R}^{2}, \mathbb{C}\right)$ such that the quadratic form

$$
Q(\psi)=\left\|\mathbb{1}_{|\cdot| \leq R_{\nu}} \nabla \psi\right\|^{2}+\frac{1}{2}\left\langle\psi,\left(V_{N}(\cdot)-M_{\nu}(\cdot)\right) \psi\right\rangle<0 .
$$

Since $V_{N}$ and $M_{v}$ are spherically symmetric we can assume that $\psi$ is spherically symmetric. Substituting $\psi \rightarrow a \psi, a \in \mathbb{R}$, we can furthermore assume that, for all

\footnotetext{
6 Note that a one particle ground state of negative energy decays exponentially, that is $\Psi_{G}(x) \leq$ $C_{1} e^{-C_{2}|x|}, C_{1}, C_{2}>0$. Hence, (131) is well defined, although $F_{v}^{Z_{n}} \notin L^{2}\left(\mathbb{R}^{2}, \mathbb{C}\right)$.
} 
$|x|=R_{\tilde{\nu}}, \psi(x)=1-\epsilon$ for $\epsilon>0$.

Define $\tilde{\psi}$ such that $\tilde{\psi}(x)=\psi(x)$ for $|x| \leq R_{v}$ and $\tilde{\psi}(x)=1$ for $|x|>R_{v}+\epsilon$ and $\epsilon>0$. Furthermore, $\tilde{\psi}$ can be constructed such that $\left\|\mathbb{1}_{|\cdot| \geq R_{\nu}} \nabla \tilde{\psi}\right\|^{2} \leq C\left(\epsilon+\epsilon^{2}\right)$.

Then $Q(\tilde{\psi})=Q(\psi)<0$ holds, because the operator associated with the quadratic form is supported inside the ball $B_{0}\left(R_{v}\right)$.

Using $\tilde{\psi}$, we can construct a set of points $Z_{n}$ and a $\chi \in H^{2}\left(\mathbb{R}^{2}, \mathbb{C}\right)$ such that $\left\langle\chi, H^{Z_{n}} \chi\right\rangle<0$, contradicting to nonnegativity of $H^{Z_{n}}$.

For $R>1$ let

$$
\xi_{R}(x)= \begin{cases}R^{2} / x^{2}, & \text { for }|x|>R \\ 1, & \text { else. }\end{cases}
$$

Let now $Z_{n}$ be a subset $Z_{n} \subset \mathbb{R}^{2}$ with $\left|Z_{n}\right|=n$ which is such that the supports of the potentials $M_{\nu}\left(\cdot-z_{k}\right)$ lie within the Ball around zero with radius $R$ and are pairwise disjoint for any two $z_{k} \in Z_{n}$. Since we are in two dimensions we can choose a $n$ which is of order $R^{2}$.

Let now $\chi_{R}(x)=\xi_{R}(x) \prod_{z_{k} \in Z_{n}} \tilde{\psi}\left(x-z_{k}\right)$. By construction, there exists a $D=\mathcal{O}(1)$ such that $\chi_{R}(x)=\tilde{\psi}\left(x-z_{k}\right)$ for $\left|x-z_{k}\right| \leq D$. From this, we obtain

$$
\begin{aligned}
\left\langle\chi_{R}, H^{Z_{n}} \chi_{R}\right\rangle & =\left\|\nabla \chi_{R}\right\|^{2}+n \frac{1}{2}\left\langle\psi,\left(V_{N}(\cdot)-M_{v}(\cdot)\right) \psi\right\rangle \\
& =n Q(\psi)+\sum_{z_{k} \in Z_{n}}\left\|\mathbb{1}_{\left|\cdot-z_{k}\right| \geq R_{v}} \nabla \chi_{R}\right\|^{2} \\
& \leq n Q(\psi)+C n\left(\epsilon+\epsilon^{2}\right)+\left\|\nabla \xi_{R}\right\|^{2} \\
& =n Q(\psi)+C n\left(\epsilon+\epsilon^{2}\right)+C .
\end{aligned}
$$

Choosing $R$ and hence $n$ large enough and $\epsilon$ small, we can find a $Z_{n}$ such that $\left\langle\chi_{R}, H^{Z_{n}} \chi_{R}\right\rangle$ is negative, contradicting nonnegativity of $H^{Z_{n}}$.

Now, we can prove that

$$
\left\|\mathbb{1}_{\left|x_{1}-x_{2}\right| \leq R_{\nu}} \nabla_{1} \Psi\right\|^{2}+\frac{1}{2}\left\langle\Psi \Psi,\left(V_{N}-M_{\nu}\right)\left(x_{1}-x_{2}\right) \Psi\right\rangle \geq 0 .
$$

holds for any $\Psi \in H^{2}\left(\mathbb{R}^{2 N}, \mathbb{C}\right)$. Using the coordinate transformation $\tilde{x}_{1}=x_{1}-$ $x_{2}, \tilde{x}_{i}=x_{i} \forall i \geq 2$, we have $\nabla_{x_{1}}=\nabla_{\tilde{x}_{1}}$. Thus (132) is equivalent to $\tilde{Q}(\Psi):=$ $\left\|\mathbb{1}_{\left|x_{1}\right| \leq R_{\nu}} \nabla_{1} \Psi\right\|^{2}+\frac{1}{2}\left\langle\Psi,\left(V_{N}-M_{\nu}\right)\left(x_{1}\right) \Psi\right\rangle \geq 0 \forall \Psi \in H^{2}\left(\mathbb{R}^{2 N}, \mathbb{C}\right)$.

If it were now that $\tilde{Q}(\Psi)$ is not nonnegative, then there exists a $\Gamma \in H^{2}\left(\mathbb{R}^{2 N}, \mathbb{C}\right)$ such that $\tilde{Q}(\Gamma)<0$. By the Schmidt decomposition theorem, there exist two orthonormal bases $\left\{\Phi_{k}\right\}_{k \in \mathbb{N}} \subset H^{2}\left(\mathbb{R}^{2 N-2}, \mathbb{C}\right),\left\{\varphi_{l}\right\}_{l \in \mathbb{N}} \subset H^{2}\left(\mathbb{R}^{2}, \mathbb{C}\right)$ and nonnegative numbers $\left\{\lambda_{k}\right\}_{k \in \mathbb{N}}$ such that

$$
\Gamma=\sum_{k \in \mathbb{N}} \lambda_{k} \varphi_{k} \otimes \Phi_{k}
$$

By this

$$
\tilde{Q}(\Gamma)=\sum_{k \in \mathbb{N}}\left|\lambda_{k}\right|^{2} Q\left(\varphi_{k}\right) \geq 0,
$$

which in turn yields to a contradictions. Therefore, $Q(\Psi) \geq 0$ for all $\Psi \in H^{2}\left(\mathbb{R}^{2}, \mathbb{C}\right)$. By a standard density argument, we can conclude that $Q(\Psi) \geq 0 \forall \Psi \in L^{2}\left(\mathbb{R}^{2 N}, \mathbb{C}\right) \cap$ $\mathcal{D}\left(\nabla_{1}\right)$. 
(b) Define $c_{k}=\left\{\left(x_{1}, \ldots, x_{N}\right) \in \mathbb{R}^{2 N}|| x_{1}-x_{k} \mid \leq R_{v}\right\}$ and $\mathcal{C}_{1}=\cup_{k=2}^{N} c_{k}$. For $\left(x_{1}, \ldots, x_{N}\right)$ $\in \mathcal{B}_{1}^{(d)}$ it holds that $\left|x_{i}-x_{j}\right| \geq N^{-d}$ for $2 \leq i, j \leq N$. Let $v>d$. Assume that $N^{-d}>2 R_{v}$, which hold for $N$ sufficiently large, since $R_{v} \leq C N^{-v}$. Then, it follows that, for $i \neq j,\left(c_{i} \cap \mathcal{B}_{1}^{(d)}\right) \cap\left(c_{j} \cap \mathcal{B}_{1}^{(d)}\right)=\emptyset$. Under the same conditions, we also have $\mathbb{1}_{\overline{\mathcal{A}}_{1}^{(d)}} \geq \mathbb{1}_{\mathcal{C}_{1}}$. Therefore

$$
\mathbb{1}_{\overline{\mathcal{A}}_{1}^{(d)}} \mathbb{1}_{\mathcal{B}_{1}^{(d)}} \geq \mathbb{1}_{\mathcal{C}_{1}} \mathbb{1}_{\mathcal{B}_{1}^{(d)}}=\mathbb{1}_{\mathcal{C}_{1} \cap \mathcal{B}_{1}^{(d)}}=\mathbb{1}_{\cup_{k=2}^{N}\left(c_{k} \cap \mathcal{B}_{1}^{(d)}\right)}=\sum_{k=2}^{N} \mathbb{1}_{c_{k} \cap \mathcal{B}_{1}^{(d)}}=\mathbb{1}_{\mathcal{B}_{1}^{(d)}} \sum_{k=2}^{N} \mathbb{1}_{c_{k}} .
$$

Note that $\mathbb{1}_{\mathcal{B}_{1}^{(d)}}$ depends only on $x_{2}, \ldots, x_{N}$. By this

$$
\left\|\mathbb{1}_{\overline{\mathcal{A}}_{1}^{(d)}} \mathbb{1}_{\mathcal{B}_{1}^{(d)}} \nabla_{1} \Psi\right\|^{2} \geq \sum_{k=2}^{N}\left\|\mathbb{1}_{c_{k}} \nabla_{1} \mathbb{1}_{\mathcal{B}_{1}^{(d)}} \Psi\right\|^{2}=(N-1)\left\|\mathbb{1}_{\left|x_{1}-x_{2}\right| \leq R_{\beta}} \nabla_{1} \mathbb{1}_{\mathcal{B}_{1}^{(d)}} \Psi\right\|^{2}
$$

This yields

$$
\begin{aligned}
(130) & \geq(N-1)\left(\left\|\mathbb{1}_{\left|x_{1}-x_{2}\right| \leq R_{\nu}} \nabla_{1} \mathbb{1}_{\mathcal{B}_{1}^{(d)}} \Psi\right\|^{2}+\frac{1}{2}\left\langle\mathbb{1}_{\mathcal{B}_{1}^{(d)}} \Psi,\left(V_{N}-M_{\nu}\right)\left(x_{1}-x_{2}\right) \mathbb{1}_{\mathcal{B}_{1}^{(d)}} \Psi\right\rangle\right) \\
& \geq 0 .
\end{aligned}
$$

where the last inequality follows from (a), using $\mathbb{1}_{\mathcal{B}_{1}^{(d)}} \Psi \in L^{2}\left(\mathbb{R}^{2 N}, \mathbb{C}\right) \cap \mathcal{D}\left(\nabla_{1}\right)$.

Lemma 7.11. Let $W_{\beta} \in \mathcal{W}_{\beta}$. Let $\Psi \in L_{s}^{2}\left(\mathbb{R}^{2 N}, \mathbb{C}\right) \cap H^{1}\left(\mathbb{R}^{2 N}, \mathbb{C}\right)$ and $\left\|\nabla_{1} \Psi\right\|$ be

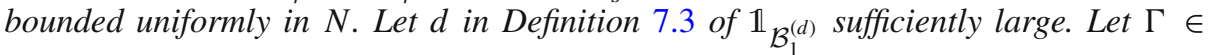
$\left\{\Psi, \mathbb{1}_{\mathcal{B}_{1}^{(d)}} \Psi\right\}$. Then, for all $\beta>0$,

(a)

$$
\begin{aligned}
& N\left|\left\langle\Gamma, q_{1} p_{2} W_{\beta}\left(x_{1}-x_{2}\right) p_{1} p_{2} \Gamma\right\rangle\right| \leq C\|\varphi\|_{\infty}^{2}\left(\langle\Psi, \hat{n} \Psi\rangle+N^{-1}\right), \\
& N \mid\left\langle\left\langle\Gamma, p_{1} q_{2} W_{\beta}\left(x_{1}-x_{2}\right) p_{1} p_{2} \Gamma\right\rangle\right| \leq C\|\varphi\|_{\infty}^{2}\left(\left\langle\langle\Psi, \hat{n} \Psi\rangle+N^{-1}\right) .\right.
\end{aligned}
$$

(b)

$$
N\left|\left\langle\Gamma, p_{1} p_{2} W_{\beta}\left(x_{1}-x_{2}\right) q_{1} q_{2} \Gamma\right\rangle\right| \leq \mathcal{K}\left(\varphi, A_{t}\right)\left(\langle\Psi, \widehat{n} \Psi\rangle+N^{-1 / 6} \ln (N)\right) .
$$

(c)

$$
N \mid\left\langle\left\langle\Gamma,\left(1-p_{1} p_{2}\right) W_{\beta}\left(x_{1}-x_{2}\right) p_{1} p_{2} \Gamma\right\rangle\right| \leq \mathcal{K}\left(\varphi, A_{t}\right)\left(\langle\Psi, \widehat{n} \Psi\rangle+N^{-1 / 6} \ln (N)\right) .
$$

Proof. (a) We will only consider the first inequality of (a). The second inequality of (a) can be proven analogously. Let first $\Gamma=\mathbb{1}_{\mathcal{B}_{1}^{(d)}} \Psi$. Then,

$$
\begin{aligned}
N \mid & \left\langle\mathbb{1}_{\mathcal{B}_{1}^{(d)}} \Psi, q_{1} p_{2} W_{\beta}\left(x_{1}-x_{2}\right) p_{1} p_{2} \mathbb{1}_{\mathcal{B}_{1}^{(d)}} \Psi\right\rangle \mid \\
\leq & N\left|\left\langle\mathbb{1}_{\overline{\mathcal{B}}_{1}^{(d)}} \Psi, q_{1} p_{2} W_{\beta}\left(x_{1}-x_{2}\right) p_{1} p_{2} \mathbb{1}_{\mathcal{B}_{1}^{(d)}} \Psi\right\rangle\right| \\
& +N\left|\left\langle\Psi \Psi, q_{1} p_{2} W_{\beta}\left(x_{1}-x_{2}\right) p_{1} p_{2} \mathbb{1}_{\mathcal{B}_{1}^{(d)}} \Psi\right\rangle\right| .
\end{aligned}
$$


Using Lemma 7.4 (c) with $\epsilon=1$, together with $\left\|p_{2} W_{\beta}\left(x_{1}-x_{2}\right) p_{2}\right\|_{\text {op }} \leq\|\varphi\|_{\infty}^{2}\left\|W_{\beta}\right\|_{1}$, the first line can be bounded by

$$
(133) \leq \mathcal{K}\left(\varphi, A_{t}\right) N\left\|\mathbb{1}_{\overline{\mathcal{B}}_{1}^{(d)}} \Psi\right\|\left\|W_{\beta}\right\|_{1} \leq \mathcal{K}\left(\varphi, A_{t}\right) N^{2-d} .
$$

The second term is bounded by

$$
\begin{aligned}
(134) & =N\left|\| \sqrt{W_{\beta}\left(x_{1}-x_{2}\right)} q_{1} p_{2}(\hat{n})^{-\frac{1}{2}} \Psi, \sqrt{W_{\beta}\left(x_{1}-x_{2}\right)} p_{1} p_{2} \hat{n}_{1}^{\frac{1}{2}} \mathbb{1}_{\mathcal{B}^{(d)} 1} \Psi\right\rangle \mid \\
& \leq C N\left\|\sqrt{W_{\beta}\left(x_{1}-x_{2}\right)} p_{2}\right\|_{\mathrm{op}}^{2}\left(\left\|q_{1}(\hat{n})^{-\frac{1}{2}} \Psi\right\|^{2}+\| \hat{n}_{1}^{\frac{1}{2}} \mathbb{1}_{\left.\mathcal{B}^{(d)}{ }_{1} \Psi \|^{2}\right)}\right. \\
& \leq C N\left\|\sqrt{W_{\beta}\left(x_{1}-x_{2}\right)} p_{2}\right\|_{\mathrm{op}}^{2}\left(\langle\Psi, \hat{n} \Psi\rangle+\left\|\hat{n}_{1}^{\frac{1}{2}} \Psi\right\|^{2}+\left\|\hat{n}_{1}^{\frac{1}{2}} \mathbb{1}_{\overline{\mathcal{B}}_{1}^{(d)}} \Psi\right\|^{2}\right) \\
& \leq C N\left\|W_{\beta}\right\|_{1}\|\varphi\|_{\infty}^{2}\left(\langle\Psi, \hat{n} \Psi\rangle+\| \mathbb{1}_{\left.\overline{\mathcal{B}}_{1}^{(d)} \Psi \|^{2}\right)}\right. \\
& \leq C\|\varphi\|_{\infty}^{2}\left(\langle\Psi, \hat{n} \Psi\rangle+N^{4-2 d}\right) \leq C\|\varphi\|_{\infty}^{2}\left(\langle\Psi \Psi, \hat{n} \Psi\rangle+N^{-1}\right) .
\end{aligned}
$$

This yields (a) in the case $\Gamma=\mathbb{1}_{\mathcal{B}_{1}^{(d)}} \Psi$. The inequality (a) can be proven analogously for $\Gamma=\Psi$.

(b) Let $\Gamma=\mathbb{1}_{\mathcal{B}_{1}^{(d)}} \Psi$. We first consider (b) for potentials with $\beta<1 / 4$. We have to estimate

$$
\begin{aligned}
& N\left|\left\langle\mathbb{1}_{\mathcal{B}_{1}^{(d)}} \Psi, p_{1} p_{2} W_{\beta}\left(x_{1}-x_{2}\right) q_{1} q_{2} \mathbb{1}_{\mathcal{B}_{1}^{(d)}} \Psi\right\rangle\right| \\
& \leq N\left|\left\langle\Psi \Psi, p_{1} p_{2} W_{\beta}\left(x_{1}-x_{2}\right) q_{1} q_{2} \Psi\right\rangle\right| \\
&+N\left|\left\langle\mathbb{1}_{\overline{\mathcal{B}}_{1}^{(d)}} \Psi, p_{1} p_{2} W_{\beta}\left(x_{1}-x_{2}\right) q_{1} q 2 \Psi\right\rangle\right| \\
&+N \mid\left\langle\left\langle\Psi, p_{1} p_{2} W_{\beta}\left(x_{1}-x_{2}\right) q_{1} q_{2} \mathbb{1}_{\overline{\mathcal{B}_{1}^{(d)}}} \Psi\right\rangle\right| \\
&+N \mid\left\langle\left\langle\mathbb{1}_{\overline{\mathcal{B}}_{1}^{(d)}} \Psi, p_{1} p_{2} W_{\beta}\left(x_{1}-x_{2}\right) q_{1} q_{2} \mathbb{1}_{\overline{\mathcal{B}_{1}^{(d)}}} \Psi\right\rangle\right| \\
& \leq N\left|\left\langle\Psi \Psi, p_{1} p_{2} W_{\beta}\left(x_{1}-x_{2}\right) q_{1} q_{2} \Psi\right\rangle\right| \\
&+C N\left\|\mathbb{1}_{\overline{\mathcal{B}}_{1}^{(d)}} \Psi\right\|\left\|W_{\beta}\right\|_{\infty} .
\end{aligned}
$$

The last term is bounded using Lemma 7.4 (c) with $\epsilon=1$

$$
(137) \leq C N N^{2-d} N^{-1+2 \beta} \leq N^{-1 / 2},
$$

where the last inequality holds choosing $d \geq 3$.

Using Lemmas 4.2 (c) and 4.6 with $O_{1,2}=\bar{q}_{2} W_{\beta}\left(x_{1}-x_{2}\right) p_{2}, \Omega=N^{-1 / 2} q_{1} \Psi$ and $\chi=N^{1 / 2} p_{1} \Psi$ we get

$$
\begin{aligned}
(136) \leq & \left\|q_{1} \Psi\right\|^{2}+N^{2} \mid\left\langle q_{2} \Psi, p_{1} \sqrt{W_{\beta}}\left(x_{1}-x_{2}\right) p_{3} \sqrt{W_{\beta}}\left(x_{1}-x_{3}\right)\right. \\
& \left.\sqrt{W_{\beta}}\left(x_{1}-x_{2}\right) p_{2} \sqrt{W_{\beta}}\left(x_{1}-x_{3}\right) p_{1} q_{3} \Psi\right\rangle \mid \\
& +N^{2}(N-1)^{-1}\left\|q_{2} W_{\beta}\left(x_{1}-x_{2}\right) p_{2} p_{1} \Psi\right\|^{2} \\
\leq & \left\|q_{1} \Psi\right\|^{2}+N^{2}\left\|\sqrt{W_{\beta}}\left(x_{1}-x_{2}\right) p_{1}\right\|_{\mathrm{op}}^{4}\left\|q_{2} \Psi\right\|^{2} \\
& +C N\left\|W_{\beta}\left(x_{1}-x_{2}\right) p_{2}\right\|_{\mathrm{op}}^{2} .
\end{aligned}
$$


With Lemma 4.2 (e) we get the bound

$$
\text { (136) } \leq\left\|q_{1} \Psi\right\|^{2}+N^{2}\|\varphi\|_{\infty}^{4}\left\|W_{\beta}\right\|_{1}^{2}\left\|q_{1} \Psi\right\|^{2}+C N\left\|W_{\beta}\right\|^{2}\|\varphi\|_{\infty}^{2} .
$$

Note, that $\left\|W_{\beta}\right\|_{1} \leq C N^{-1},\left\|W_{\beta}\right\|^{2} \leq C N^{-2+2 \beta}$. Hence

$$
(136) \leq C\left(\left\langle\Psi, q_{1} \Psi\right\rangle+\mathcal{K}\left(\varphi, A_{t}\right) N^{-1+2 \beta}\right) .
$$

Note that, for $\beta<1 / 4, N^{-1+2 \beta} \leq N^{-1 / 6} \ln (N)$. Using the same bounds for $\Gamma=\Psi$, we obtain (b) for the case $\beta<1 / 4$.

b) for $1 / 4 \leq \beta$ :

We use $\bar{U}_{\beta_{1}, \beta}$ from Definition 7.1 for some $0<\beta_{1}<1 / 4$.

By the estimate above, it is left to control

$$
N\left|\left\langle\mathbb{1}_{\mathcal{B}_{1}^{(d)}} \Psi, p_{1} p_{2}\left(W_{\beta}\left(x_{1}-x_{2}\right)-U_{\beta_{1}, \beta}\left(x_{1}-x_{2}\right)\right) q_{1} q_{2} \mathbb{1}_{\mathcal{B}_{1}^{(d)}} \Psi\right\rangle\right| .
$$

Let $\Delta h_{\beta_{1}, \beta}=W_{\beta}-U_{\beta_{1}, \beta}$. Integrating by parts and using that $\nabla_{1} h_{\beta_{1}, \beta}\left(x_{1}-x_{2}\right)=-\nabla_{2} h_{\beta_{1}, \beta}\left(x_{1}-x_{2}\right)$ gives

$$
\begin{aligned}
N \mid & \left\langle\mathbb{1}_{\mathcal{B}_{1}^{(d)}} \Psi, p_{1} p_{2}\left(W_{\beta}\left(x_{1}-x_{2}\right)-U_{\beta_{1}, \beta}\left(x_{1}-x_{2}\right)\right) q_{1} q_{2} \mathbb{1}_{\mathcal{B}_{1}^{(d)}} \Psi\right\rangle \mid \\
= & N\left|\left\langle\nabla_{1} p_{1} \mathbb{1}_{\mathcal{B}_{1}^{(d)}} \Psi, p_{2} \nabla_{2} h_{\beta_{1}, \beta}\left(x_{1}-x_{2}\right) q_{1} q_{2} \mathbb{1}_{\mathcal{B}_{1}^{(d)}} \Psi\right\rangle\right| \\
& +N\left|\left\langle\mathbb{1}_{\mathcal{B}_{1}^{(d)}} \Psi, p_{1} p_{2} \nabla_{2} h_{\beta_{1}, \beta}\left(x_{1}-x_{2}\right) \nabla_{1} q_{1} q_{2} \mathbb{1}_{\mathcal{B}_{1}^{(d)}} \Psi\right\rangle\right| .
\end{aligned}
$$

Let $\left(a_{1}, b_{1}\right)=\left(q_{1}, \nabla p_{1}\right)$ or $\left(a_{1}, b_{1}\right)=\left(\nabla q_{1}, p_{1}\right)$. Then, both terms can be estimated as follows:

We use Lemma 4.6 with $\Omega=N^{-\eta / 2} a_{1} \mathbb{1}_{\mathcal{B}_{1}^{(d)}} \Psi, O_{1,2}=N^{1+\eta / 2} q_{2} \nabla_{2} h_{\beta_{1}, \beta}\left(x_{1}-\right.$ $\left.x_{2}\right) p_{2}$ and $\chi=b_{1} \mathbb{1}_{\mathcal{B}_{1}^{(d)}} \Psi$. We choose $\eta<2 \beta_{1}$.

$$
\begin{aligned}
N \mid & \left\langle\mathbb{1}_{\mathcal{B}_{1}^{(d)}} \Psi, a_{1} p_{2} \nabla_{2} h_{\beta_{1}, \beta}\left(x_{1}-x_{2}\right) b_{1} q_{2} \mathbb{1}_{\mathcal{B}_{1}^{(d)}} \Psi\right\rangle \mid \\
\leq & N^{-\eta}\left\|a_{1} \mathbb{1}_{\mathcal{B}_{1}^{(d)}} \Psi\right\|^{2} \\
& +\frac{N^{2+\eta}}{N-1}\left\|q_{2} \nabla_{2} h_{\beta_{1}, \beta}\left(x_{1}-x_{2}\right) b_{1} p_{2} \mathbb{1}_{\mathcal{B}_{1}^{(d)}} \Psi\right\|^{2} \\
& +N^{2+\eta}\left|\left\langle\mathbb{1}_{\mathcal{B}_{1}^{(d)}} \Psi, b_{1} p_{2} q_{3} \nabla_{2} h_{\beta_{1}, \beta}\left(x_{1}-x_{2}\right) \nabla_{3} h_{\beta_{1}, \beta}\left(x_{1}-x_{3}\right) b_{1} q_{2} p_{3} \mathbb{1}_{\mathcal{B}_{1}^{(d)}} \Psi\right\rangle\right|^{1 / 2} .
\end{aligned}
$$

We obtain (note that $\mathbb{1}_{\mathcal{B}_{1}^{(d)}}$ does not depend on $x_{1}$ )

$$
(140) \leq N^{-\eta}\left\|a_{1} \mathbb{1}_{\mathcal{B}_{1}^{(d)}} \Psi\right\|^{2}=N^{-\eta}\left\|\mathbb{1}_{\mathcal{B}_{1}^{(d)}} a_{1} \Psi\right\|^{2} \leq \mathcal{K}\left(\varphi, A_{t}\right) N^{-\eta}
$$

since both $\left\|\nabla q_{1} \Psi\right\|$ and $\left\|q_{1} \Psi\right\|$ are bounded uniformly in $N$. Since $q_{2}$ is a projector it follows that

$$
\begin{aligned}
(141) & \leq \frac{N^{2+\eta}}{N-1}\left\|\nabla_{2} h_{\beta_{1}, \beta}\left(x_{1}-x_{2}\right) p_{2}\right\|_{\mathrm{op}}^{2}\left\|b_{1} \mathbb{1}_{\mathcal{B}_{1}^{(d)}} \Psi\right\|^{2} \\
& \leq C \frac{N^{2+\eta}}{N-1}\|\varphi\|_{\infty}^{2}\left\|\nabla h_{\beta_{1}, \beta}\right\|^{2}\left\|b_{1} \mathbb{1}_{\mathcal{B}_{1}^{(d)}} \Psi\right\|^{2} \\
& \leq \mathcal{K}\left(\varphi, A_{t}\right) N^{\eta-1} \ln (N)\|\varphi\|_{\infty}^{2},
\end{aligned}
$$


where we used Lemma 7.2 in the last step.

Next, we estimate

$$
\begin{aligned}
(142) \leq & N^{2+\eta}\left\|p_{2} \nabla_{2} h_{\beta_{1}, \beta}\left(x_{1}-x_{2}\right) b_{1} q_{2} \mathbb{1}_{\mathcal{B}_{1}^{(d)}} \Psi\right\|^{2} \\
\leq & 2 N^{2+\eta}\left\|p_{2} \nabla_{2} h_{\beta_{1}, \beta}\left(x_{1}-x_{2}\right) b_{1} q_{2} \mathbb{1}_{\overline{\mathcal{B}}_{1}^{(d)}} \Psi\right\|^{2} \\
& +2 N^{2+\eta}\left\|p_{2} \nabla_{2} h_{\beta_{1}, \beta}\left(x_{1}-x_{2}\right) b_{1} q_{2} \Psi\right\|^{2} .
\end{aligned}
$$

The first term can be estimated as

$$
\begin{aligned}
(143) & \leq C N^{2+\eta}\left\|\nabla_{2} h_{\beta_{1}, \beta}\left(x_{1}-x_{2}\right) b_{1}\right\|_{\mathrm{op}}^{2}\left\|\mathbb{1}_{\overline{\mathcal{B}}_{1}^{(d)}} \Psi\right\|^{2} \\
& \leq C N^{2+\eta}\left\|\nabla_{2} h_{\beta_{1}, \beta}\right\|^{2}\left(\|\varphi\|_{\infty}^{2}+\|\nabla \varphi\|_{\infty}^{2}\right)\left\|\mathbb{1}_{\overline{\mathcal{B}}_{1}^{(d)}} \Psi\right\|^{2} \\
& \leq \mathcal{K}\left(\varphi, A_{t}\right) N^{2+\eta} N^{-2} \ln (N) N^{4-2 d} \\
& \leq \mathcal{K}\left(\varphi, A_{t}\right) N^{-2+\eta} \ln (N),
\end{aligned}
$$

for any $d \geq 3$. In the last line we used Lemma 7.4 (c) with $\epsilon=1$. The last term can be estimated as

$$
\begin{aligned}
(144) \leq & 2 N^{2+\eta}\left\|p_{2} h_{\beta_{1}, \beta}\left(x_{1}-x_{2}\right) b_{1} \nabla_{2} q_{2} \Psi\right\|^{2} \\
& +2 N^{2+\eta} \|\left|\varphi\left(x_{2}\right)\right\rangle\left\langle\nabla \varphi\left(x_{2}\right)\right| h_{\beta_{1}, \beta}\left(x_{1}-x_{2}\right) b_{1} q 2 \Psi \|^{2} \\
\leq & C N^{2+\eta}\left\|p_{2} h_{\beta_{1}, \beta}\left(x_{1}-x_{2}\right)\right\|_{\mathrm{op}}^{2}\left\|b_{1} \nabla_{2} q_{2} \Psi\right\|^{2} \\
& +C N^{2+\eta} \|\left|\varphi\left(x_{2}\right)\right\rangle\left\langle\nabla \varphi\left(x_{2}\right)\right| h_{\beta_{1}, \beta}\left(x_{1}-x_{2}\right)\left\|_{\mathrm{op}}^{2}\right\| b_{1} q q_{2} \Psi \|^{2} \\
\leq & C N^{2+\eta}\left(\|\nabla \varphi\|_{\infty}^{2}+\|\varphi\|_{\infty}^{2}\right)\left\|h_{\beta_{1}, \beta}\right\|^{2}\left(1+\|\nabla \varphi\|^{2}\right) \\
\leq & \mathcal{K}\left(\varphi, A_{t}\right) N^{\eta-2 \beta_{1}} \ln (N)^{2} .
\end{aligned}
$$

Combining both estimates we obtain, for any $\beta>1 / 4$,

$$
\begin{aligned}
N \mid & \left\langle\mathbb{1}_{\mathcal{B}_{1}^{(d)}} \Psi, p_{1} p_{2} W_{\beta}\left(x_{1}-x_{2}\right) q_{1} q_{2} \mathbb{1}_{\mathcal{B}^{(d)}{ }_{1}} \Psi\right\rangle \mid \\
\leq & \inf _{\eta>0} \inf _{0<\beta_{1}<1 / 4}\left(\mathcal { K } ( \varphi , A _ { t } ) \left(\langle\Psi, \widehat{n} \Psi\rangle+N^{-1+2 \beta_{1}}+N^{-\eta}\right.\right. \\
& \left.\left.+N^{\eta-1} \ln (N)+N^{\eta-2 \beta_{1}} \ln (N)\right)\right) \\
\leq & \mathcal{K}\left(\varphi, A_{t}\right)\left(\langle\Psi, \widehat{n} \Psi\rangle+N^{-1 / 6} \ln (N)\right) .
\end{aligned}
$$

where the last inequality comes from choosing $\eta=1 / 3$ and $\beta_{1}=1 / 4$. For $\Gamma=\Psi$, (b) can be estimated the same way, yielding the same bound.

(c) This follows from (a) and (b), using that $1-p_{1} p_{2}=q_{1} q_{2}+p_{1} q_{2}+q_{1} p_{2}$.

Acknowledgements Open access funding provided by Institute of Science and Technology (IST Austria). We are grateful to David Mitrouskas for many valuable discussions and would like to thank Serena Cenatiempo for helpful discussions. We also would like to thank an anonymous referee for various helpful remarks which particularly improved the presentation of the paper. M.J. gratefully acknowledges financial support by the German National Academic Foundation. N.L. gratefully acknowledges financial support by the Cusanuswerk and the European Research Council (ERC) under the European Union's Horizon 2020 research and innovation programme (Grant Agreement No. 694227). P.P. gratefully acknowledges funding by the Deutsche Forschungsgemeinschaft (DFG), Grant No. PI 1114/3-1. 
Open Access This article is distributed under the terms of the Creative Commons Attribution 4.0 International License (http://creativecommons.org/licenses/by/4.0/), which permits unrestricted use, distribution, and reproduction in any medium, provided you give appropriate credit to the original author(s) and the source, provide a link to the Creative Commons license, and indicate if changes were made.

Publisher's Note Springer Nature remains neutral with regard to jurisdictional claims in published maps and institutional affiliations.

\section{References}

1. Anapolitanos, I., Hott, M.: A simple proof of convergence to the Hartree dynamics in Sobolev trace norms. J. Math. Phys. 57, 122108 (2016)

2. Ammari, Z., Falconi, M., Pawilowski, B.: On the rate of convergence for the mean field approximation of bosonic many-body quantum dynamics. Commun. Math. Sci. 14(5), 1417-1442 (2016)

3. Bagnato, V., Kleppner, D.: Bose-Einstein condensation in low-dimensional traps. Phys. Rev. A 44, 7439 (1991)

4. Ben Abdallah, N., Méhats, F., Pinaud, O.: Adiabatic approximation of the Schrödinger-Poisson system with a partial confinement. SIAM J. Math. Anal. 36(3), 986-1013 (2005)

5. Boccato, C., Cenatiempo, S., Schlein, B.: Quantum many-body fluctuations around nonlinear Schrödinger dynamics. Ann. Henri Poincaré 18, 113-191 (2017)

6. Boßmann, L.: Derivation of the $1 \mathrm{~d}$ nonlinear Schrödinger equation from the $3 \mathrm{~d}$ quantum many-body dynamics of strongly confined bosons. J. Math. Phys. 60(3), 031902 (2019)

7. Boßmann, L.: Derivation of the 2d Gross-Pitaevskii Equation for Strongly Confined 3d Bosons (2019). arXiv: 1907.04547

8. Boßmann, L., Teufel, S.: Derivation of the 1d Gross-Pitaevskii equation from the $3 \mathrm{~d}$ quantum many-body dynamics of strongly confined bosons. Ann. Henri Poincaré 20(3), 1003-1049 (2019)

9. Benedikter, N., De Oliviera, G., Schlein, B.: Quantitative derivation of the Gross-Pitaevskii equation. Commun. Pure Appl. Math. 68(8), 1399-1482 (2015)

10. Benedikter, N., Porta, M., Schlein, B.: Effective Evolution Equations from Quantum Dynamics, Springer Briefs in Mathematical Physics Cambridge, ISBN 978-3-319-24898-1 (2016)

11. Brennecke, C., Schlein, B.: Gross-Pitaevskii dynamics for Bose-Einstein condensates. Anal. PDE 12(6), 1513-1596 (2019)

12. Brennecke, C., Nam, P.T., Napiórkowski, M., Schlein, B.: Fluctuations of N-Particle Quantum Dynamics Around the Nonlinear Schrödinger Equation (2017). arXiv:1710.09743

13. Carles, R., Silva, J.: Drumond: Large time behavior in nonlinear Schrodinger equation with time dependent potential. Commun. Math. Sci. 13(2), 443-460 (2015)

14. Chen, X., Holmer, J.: The rigorous derivation of the $2 \mathrm{~d}$ cubic focusing NLS from quantum many-body evolution. Int. Math. Res. IMRN 14, 4173-4216 (2017)

15. Chen, X., Holmer, J.: On the rigorous derivation of the $2 \mathrm{~d}$ cubic nonlinear Schrödinger equation from $3 \mathrm{~d}$ quantum many-body dynamics. Arch. Ration. Mech. Anal. 210(3), 909-954 (2013)

16. Chen, X., Holmer, J.: Focusing quantum many-body dynamics: the rigorous derivation of the $1 \mathrm{~d}$ focusing cubic nonlinear Schrödinger equation. Arch. Ration. Mech. Anal. 221(2), 631-676 (2016)

17. Chen, X., Holmer, J.: Focusing quantum many-body dynamics II: the rigorous derivation of the $1 \mathrm{~d}$ focusing cubic nonlinear Schrödinger equation from 3d. Anal. PDE 10(3), 583-633 (2017)

18. Cherny, AYu., Shanenko, A.A.: Dilute Bose gas in two dimensions: density expansions and the GrossPitaevskii equation. Phys. Rev. E 64, 027105 (2001)

19. Erdös, L., Schlein, B., Yau, H.-T.: Derivation of the Gross-Pitaevskii hierarchy for the dynamics of Bose-Einstein condensate. Commun. Pure Appl. Math. 59(12), 1659-1741 (2006)

20. Erdös, L., Schlein, B., Yau, H.-T.: Derivation of the cubic non-linear Schrödinger equation from quantum dynamics of many-body systems. Invent. Math. 167, 515-614 (2007)

21. Erdös, L., Schlein, B., Yau, H.-T.: Derivation of the Gross-Pitaevskii equation for the dynamics of BoseEinstein condensate. Ann. Math. (2) 172(1), 291-370 (2010)

22. Erdös, L., Schlein, B., Yau, H.-T.: Rigorous derivation of the Gross-Pitaevskii equation with a larger interaction potential. J. Am. Math. Soc. 22(4), 1099-1156 (2009)

23. Grillakis, M., Machedon, M.: Pair excitations and the mean field approximation of interacting Bosons I. Commun. Math. Phys. 324, 601-636 (2013)

24. Grillakis, M., Machedon, M.: Pair excitations and the mean field approximation of interacting Bosons II. Commun. Partial Differ. Equ. 42, 24-67 (2017)

25. Grillakis, M., Machedon, M.: Second-order corrections to mean field evolution of weakly interacting bosons. II. Adv. Math. 228, 1788-1815 (2011) 
26. Jeblick, M., Pickl, P.: Derivation of the time dependent two dimensional focusing NLS equation. J. Stat. Phys. 172(5), 1398-1426 (2018)

27. Kirkpatrick, K., Schlein, B., Staffilani, G.: Derivation of the two-dimensional nonlinear Schrödinger equation from many body quantum dynamics. Am. J. Math. 133(1), 91-130 (2011)

28. Keler, J.V.: Mean Field Limits in Strongly Confined Systems (2014). arXiv:1412.3437

29. Keler, J.V., Teufel, S.: The NLS Limit for bosons in a quantum waveguide. Ann. Henri Poincaré 17(12), 3321-3360 (2016)

30. Knowles, A., Pickl, P.: Mean-field dynamics: singular potentials and rate of convergence. Commun. Math. Phys. 298, 101-139 (2010)

31. Kuz, E.: Exact evolution versus mean field with second-order correction for Bosons interacting via shortrange two-body potential. Differe. Integral Equ. 30, 587-630 (2017)

32. Leopold, N., Pickl, P.: Mean-field limits of particles in interaction with quantized radiation fields. In: Macroscopic Limits of Quantum Systems, Springer Proceedings in Mathematics \& Statistics, pp. 185214. Springer, Berlin (2018)

33. Leopold, N., Petrat, S.: Mean-field dynamics for the Nelson model with Fermions. Ann. Henri Poincaré (2019). https://doi.org/10.1007/s00023-019-00828-w

34. Lewin, M., Nam, P.T., Rougerie, N.: A note on 2D focusing many-boson systems. Proc. Am. Math. Soc. 145(6), 2441-2454 (2017)

35. Lewin, M., Nam, P.T., Schlein, B.: Fluctuations around Hartree states in the mean-field regime. Am. J. Math. 137, 1613-1650 (2015)

36. Lieb, E., Loss, M.: Analysis. Graduate Studies in Mathematics. American Mathematical Society, Providence (2010)

37. Lieb, E., Seiringer, R.: Proof of Bose-Einstein condensation for dilute trapped gases. Phys. Rev. Lett. 88, 170409 (2002)

38. Lieb, E.H., Seiringer, R., Solovej, J.P., Yngvason, J.: The Mathematics of the Bose Gas and Its Condensation, Oberwolfach Seminars, vol. 34. Birkhauser Verlag, Basel (2005)

39. Lieb, E.H., Seiringer, R., Yngvason, J.: A rigorous derivation of the Gross-Pitaevskii energy functional for a two-dimensional Bose gas. Commun. Math. Phys. 224, 17 (2001)

40. Lieb, E.H., Yngvason, J.: The ground state energy of a dilute two-dimensional Bose gas. J. Stat. Phys. 103, 509 (2001)

41. Michelangeli, A.: Equivalent definitions of asymptotic $100 \%$ BEC. Nuovo Cimento Sec. B. 123, 181-192 (2008)

42. Mitrouskas, D., Petrat, S., Pickl, P.: Bogoliubov corrections and trace norm convergence for the Hartree dynamics. Rev. Math. Phys. 31(8), 1950024 (2019)

43. Nam, P.T., Napiórkowski, M.: A note on the validity of Bogoliubov correction to mean-field dynamics. J. Math. Pure Appl. 108, 662-688 (2017)

44. Nam, P.T., Napiórkowski, M.: Bogoliubov correction to the mean-field dynamics of interacting bosons. Adv. Theor. Math. Phys. 21, 683-738 (2017)

45. Nam, P.T., Napiórkowski, M.: Norm approximation for many-body quantum dynamics: focusing case in low dimensions. Adv. Math. 350, 547-587 (2019)

46. Mitrouskas, D., Petrat, S., Pickl, P.: Bogoliubov corrections and trace norm convergence for the Hartree dynamics. Rev. Math. Phys. 31(8), 1950024 (2019)

47. Petrat, S., Pickl, P.: A new method and a new scaling for deriving fermionic mean-field dynamics. Math. Phys. Anal. Geom. 19(1), 3 (2016)

48. Pickl, P.: Derivation of the time dependent Gross-Pitaevskii equation without positivity condition on the interaction. J. Stat. Phys. 140, 76-89 (2010)

49. Pickl, P.: Derivation of the time dependent Gross-Pitaevskii equation with external fields. Rev. Math. Phys. 27(1), 1550003 (2015)

50. Pickl, P.: A simple derivation of mean field limits for quantum systems. Lett. Math. Phys. 97, 151-164 (2011)

51. Rodnianski, I., Schlein, B.: Quantum fluctuations and rate of convergence towards mean field dynamics. Commun. Math. Phys. 291(1), 31-61 (2009)

52. Teschl, G.: Mathematical Methods in Quantum Mechanics With Applications to Schrödinger Operators, Graduate Studies in Mathematics, vol. 157. American Mathematical Society, Providence (2014) 